\title{
Exploiting NMR Spectroscopy for the Study of Disorder in Solids
}

Robert F. Moran, Daniel M. Dawson and Sharon E. Ashbrook*

School of Chemistry, EaStCHEM and St Andrews Centre of Magnetic Resonance, University of St Andrews, North Haugh, St Andrews KY16 9ST, UK

*Author to whom correspondence should be addressed.

E-mail: sema@st-andrews.ac.uk

Invited article for Int. Rev. Phys. Chem. 


\begin{abstract}
Although the solid state is typically characterised by inherent periodicity, many interesting physical and chemical properties of solids arise from a variation in this, i.e., changes in the nature of the atom occupying a particular site in a crystal structure or variation in the position of an atom (or group of atoms) in different parts of a structure, or variation as a function of time. This lack of long-range order poses significant challenges, not just for the characterisation of the structure of disordered materials, but also simply for its description. The sensitivity of nuclear magnetic resonance (NMR) spectroscopy to the local, atomic-scale environment, without the requirement for long-range order, makes it a powerful tool for the study of disorder in the solid state. Information on the number and type(s) of coordinating atoms or through-space and through-bond connectivity between atomic species enables the construction of a detailed picture of the structure. After a brief description of the background theory of NMR spectroscopy, and the experimental methods employed, we will describe the effects of disorder on NMR spectra and the use of calculations to help interpret experimental measurements. We will then review a range of applications to different types of disordered materials, including oxides and ceramics, minerals, porous materials, biomaterials, energy materials, pharmaceuticals, polymers and glasses. We will discuss the most successful approaches for studying different materials, and illustrate the type of information available and the structural insight gained.
\end{abstract}

\title{
Keywords
}

solid-state, NMR spectroscopy, disordered materials, DFT calculations, inorganic solids 


\section{Introduction}

Although the solid state is typically characterised by long-range order or periodicity, many of the more interesting physical and chemical properties of solids arise from a variation in this, i.e., from some form of disorder. Variations in composition or atomic positions can lead to significant changes in the properties of a material, e.g., increasing ionic or electrical conductivity, improving chemical, mechanical or thermal stability or enabling ferroelectric or magnetic behaviour. The sensitivity of many properties to even very small changes in composition or structure, enables a system to be "tuned" to obtain the desired behaviour under specific conditions, providing a variety of applications of disordered materials in the energy arena, in the electronics industry, for construction, as catalysts, for waste storage and in the pharmaceutical industry.

The characterisation of solid-state structure is typically dominated by methods based on Bragg diffraction. For ideal solids (such as that shown schematically in Figure 1a), the long-range order in both atomic positions and site occupancies enables diffraction to produce a complete and accurate structure solution from single-crystal measurements or, in some cases, from powder data. However, any variation in periodicity hinders this procedure, with diffraction then able only to provide information on the "average" structure. For example, Figure $1 b$ shows a schematic crystal structure where the periodicity of the atomic positions is retained, but the atom occupying one of the crystallographic sites varies. In this case of compositional disorder, it is typical for a structure refinement to determine the atomic coordinates precisely, but to define a site as having a fractional occupancy, e.g., $\mathrm{B}_{0.5} \mathrm{C}_{0.5}$ for the second atom in Figure $1 \mathrm{~b}$. While this nomenclature indicates that two atom types are found at a particular site in the structure, it does not provide any information on how the two might be distributed over these sites whether this distribution is random, or whether any clustering or preferred avoidance occurs. In other cases, as shown schematically in Figure 1c, the position of atoms or groups of atoms (e.g., $\mathrm{OH}^{-}, \mathrm{H}_{2} \mathrm{O}, \mathrm{F}^{-}$, etc.,) varies, resulting in positional or topological disorder. This can also be described by fractional occupancies, now formally between an atom and a 
vacancy. For example, in Figure 1c the A sites have a fractional occupancy of 0.75 (indicating each is vacant in $25 \%$ of cases). However, some caution is needed here as, more generally, it is not clear from a fractional occupancy whether a site is occupied in $25 \%$ of the material (as in Figure 1c) or for $25 \%$ of the time, i.e., a dynamic rather than a static disorder. Significant positional variation (be it static or dynamic) may result in atoms or molecules not being located at all in the structure solution. This is often the case for water or solvent molecules in pharmaceuticals, minerals or microporous materials. In addition to fractional occupancies, it may be that variations in local geometry, i.e., bond distances and bond angles are present in a material. As this becomes more significant, the definition of a "crystal structure" becomes less clear, posing significant challenges for diffraction-based approaches. This is the case for amorphous materials, such as glasses, as shown in Figure 1d, where specific "sites" can no longer be defined and, while the local geometry around a type of atom is often very similar, the long-range structure is now very different.

As the level and nature of disorder increases, Bragg diffraction (and perhaps even the idea of "the crystal structure" itself) becomes less useful. In order to fully describe the structure of a disordered solid it is necessary to consider not only any periodic aspects of the structure that are retained, but also the changes in local geometry, e.g., the number and type of coordinating atoms, the nature of species on the next nearest neighbour (NNN) sites and the variation in the bonding geometry. In this respect, nuclear magnetic resonance (NMR) spectroscopy provides an ideal (and complementary) tool, with the chemical shift a very sensitive probe of small changes in the local environment, and couplings between nuclear spins (both through bond and through space) able to provide information on covalent bonding and spatial proximities.[1] This has resulted in NMR spectroscopy becoming one of the most widely-used analytical tools in the chemical sciences, with widespread applications in many fields. However, unlike their solutionstate counterparts, NMR spectra of solids often contain broad and featureless lineshapes as a result of the anisotropic (i.e., orientation-dependent) nature of the interactions that affect the nuclear spins - interactions that are averaged in solution by the rapid tumbling motion.[2-4] There are a number of approaches for removing anisotropic broadening and 
improving resolution, some involving physical manipulation of the sample, such as magic-angle spinning (MAS),[5] others just a manipulation of the nuclear spins, such as decoupling. The spectra of nuclei with higher spin quantum number (i.e., I > 1/2) are also affected by an interaction with the electric field gradient (the quadrupolar interaction), resulting in a broadening that cannot be removed by MAS and more complex techniques are required to obtain high-resolution spectra.[1,6-8]

Even when high-resolution approaches are used, solid-state NMR spectra may still contain complicated or overlapped spectral lineshapes, particularly as the structural complexity and/or level of disorder in a material increases. This hinders resolution, and it can be difficult both to assign the spectral resonances and to extract accurately the structural information available. In general, NMR spectra of solids are more complicated than those from solution-state experiments as the crystal packing in a solid produces not only chemically- but also crystallographically-distinct species. Although, for molecular solids, some insight can be gained through comparison to solution-state NMR spectra, this does not help assign resonances from sites rendered inequivalent only by the packing, which have very similar chemical environments and, therefore, very similar NMR parameters. For many extended inorganic solids there is no molecular solution-state counterpart to provide insight, and typically a range of less commonly-studied nuclear species are investigated (many of which have inherently low natural abundance or low sensitivity) and with, often, relatively little information in the literature to aid spectral interpretation. Additional information can be obtained by measuring the magnitude of the NMR parameters (both isotropic and anisotropic) although, in the latter case, this can require recoupling (i.e., a selective reintroduction of interactions removed by techniques such as MAS), or by the use of experiments that transfer magnetisation between nuclear spins, providing information on through-bond and through-space interactions.

The challenge in assigning and interpreting solid-state NMR spectra (and directly producing a similar sort of structural picture to that available from diffraction) has resulted in recent years in growing interest in the use of first-principles calculations to 
predict NMR parameters for a structure or structural model.[9-11] Although well established for molecular systems, with a variety of codes available, such approaches have only become widespread in the solid-state NMR community since the advent of approaches able to calculate NMR parameters for periodic systems, i.e., exploiting the inherent transitional symmetry of the solid state.[12] For ordered crystalline solids, calculations can be used to assign well-defined spectral resonances, to confirm unexpected NMR parameters, or to predict the magnitude of parameters that are difficult to extract experimentally (including anisotropic interactions or tensor orientations), in many cases guiding experimental measurements or analytical fitting procedures. Calculations often play a vital role in the emerging field of NMR crystallography (the use of NMR spectroscopy, often in combination with diffraction experiments, to solve or refine structures).[13,14] For disordered materials, the computational challenge is much greater, with the deviation from periodicity often requiring a large number of different calculations to be performed and/or larger supercells employed. However, the ability to predict NMR parameters for species with varying local and long-range environments provides insight into the interpretation of the complex spectral lineshapes and important information about the local structural environments present.

In this review, we will discuss the use of solid-state NMR spectroscopy to investigate the structure of disordered materials. After a brief description of the background theory and the experimental methods employed, we will describe the effects of disorder on NMR spectra and the use of calculations to help interpret experimental measurements. We will then review a range of applications to different types of disordered materials, including oxides and ceramics, minerals, porous materials, biomaterials, energy materials, pharmaceuticals, polymers and glasses. Clearly, this cannot be a comprehensive review of each area, but we hope to discuss the most important considerations and most successful approaches for different materials, and to illustrate the type of information available and the insight gained by discussing some selected case studies in more detail. For further information on each area, readers are referred to the more comprehensive reviews highlighted in later sections. 


\section{Basics of Solid-State NMR}

\subsection{Magnetisation and Pulses}

NMR spectroscopy is concerned with the interaction of electromagnetic radiation with the intrinsic magnetic moments of nuclei. The nuclear magnetism arises from the quantummechanical nuclear spin, I, (where the bold typeface represents a vector quantity) described by the quantum number, I. As I arises from the combined spins of the protons and neutrons (both of which have I = 1/2), I may take any integer or half-integer value. The nuclear magnetic moment, $\boldsymbol{\mu}$, is given by

$$
\boldsymbol{\mu}=\gamma \mathbf{I}
$$

where $\gamma$ is the gyromagnetic ratio, characteristic of a given nuclide. As shown in Figure 2a, in an external magnetic field, $\mathbf{B}_{0}$, (magnitude $\mathbf{B}_{0}$ ), the magnetic moments are quantised along the field direction (by definition the $\mathrm{z}$ axis) in units of $\hbar$, with

$$
\mu_{\mathrm{z}}=\gamma \mathrm{m}_{\mathrm{I}} \hbar
$$

where $m_{I}$ is the magnetic quantum number, which can take any integer or half-integer value between $-\mathrm{I}$ and $+\mathrm{I}$. The energy of each spin state (the Zeeman energy) is given by $\mu_{\mathrm{z}} \mathrm{B}_{0}$ and the energy difference, $\Delta \mathrm{E}$, between two states is, therefore,

$$
\Delta \mathrm{E}=\Delta \mathrm{m}_{\mathrm{I}} \gamma \hbar \mathrm{B}_{0}
$$

As only single-quantum transitions $\left(\Delta \mathrm{m}_{\mathrm{I}}= \pm 1\right)$ are allowed by the NMR selection rules, all observable transitions occur with $\Delta \mathrm{E}=\gamma \hbar \mathrm{B}_{0}$, corresponding to a frequency (termed the Larmor frequency) of $\omega_{0}=-\gamma B_{0}$ in rad s$s^{-1}$ or $v_{0}=\omega_{0} / 2 \pi$ in Hz. Typical $B_{0}$ fields used range from 4 to $24 \mathrm{~T}$, giving rise to Larmor frequencies corresponding to the radiofrequency (rf) region of the spectrum, on the order of $\sim 5-1000 \mathrm{MHz}$.

In a macroscopic ensemble of spins, the Zeeman states are populated according to a Boltzmann distribution, with

$$
\frac{\mathrm{N}_{\mathrm{m}_{\mathrm{I}}+1}}{\mathrm{~N}_{\mathrm{m}_{\mathrm{I}}}}=\mathrm{e}^{-\gamma \hbar \mathrm{B}_{0} / \mathrm{k}_{\mathrm{B}} \mathrm{T}},
$$


where $\mathrm{N}$ is the population of the corresponding $\mathrm{m}_{\mathrm{I}}$ state, $\mathrm{k}_{\mathrm{B}}$ is Boltzmann's constant and $\mathrm{T}$ is the absolute temperature. For $I=1 / 2$ nuclei, the population difference between the two spin states $\left(m_{I}= \pm 1 / 2\right)$ gives rise to a bulk magnetisation, $\mathbf{M}$, which can be considered as the observable in an NMR experiment, as shown in Figure 2. As the population difference is typically on the order of a few spins in $10^{5}$ to $10^{7}, \mathbf{M}$ is very small and NMR spectroscopy is inherently insensitive, leading to considerable interest in approaches for improving sensitivity in many aspects of the technique. At thermal equilibrium, $\mathbf{M}$ is aligned along $\mathrm{z}$ and precesses about this axis with frequency, $\omega_{0}$. The magnetisation can be manipulated by the application of short bursts of rf irradiation, or "pulses" with frequency, $\omega_{\mathrm{rf}} \approx \omega_{0}$. The effect of a pulse is most easily considered in the rotating frame $-\mathrm{a}$ coordinate system in which the $\mathrm{z}$ axis remains aligned with the laboratory $\mathrm{z}$ axis (defined by $\mathbf{B}_{0}$ ) and the xy plane rotates about the $\mathrm{z}$ axis at $\omega_{\mathrm{rf}}$. In this rotating frame, and as shown in Figure $2 b$, the pulse appears as a static field, $\mathbf{B}_{1}$, (magnitude $\mathbf{B}_{1}$ ), in the xy plane and $\mathbf{M}$ now precesses about $\mathrm{z}$ at an offset frequency, $\Omega$, where

$$
\Omega=\omega_{0}-\omega_{\mathrm{rf}}
$$

which then implies an effective field along $\mathrm{z}$ of

$$
\mathrm{B}_{\mathrm{eff}}=\Omega / \gamma \text {. }
$$

When a pulse is applied "on resonance" $\omega_{\mathrm{rf}}=\omega_{0}, \mathrm{~B}_{\mathrm{eff}}=0$ and $\mathbf{M}$ nutates about $\mathbf{B}_{1}$ at a frequency

$$
\omega_{1}=-\gamma B_{1} \text {. }
$$

Over the duration of the pulse, $\tau_{\mathrm{p}}$ M will nutate through a "flip angle",

$$
\beta=\omega_{1} \tau_{p} \text {. }
$$

Once the $B_{1}$ field is removed, $\mathbf{M}$ will again precess about $\mathrm{z}$ at frequency $\Omega$, but now has a component in the xy plane. Over time, $\mathbf{M}$ will return to equilibrium through spin-lattice or longitudinal relaxation, described by the time constant, $\mathrm{T}_{1}$, typically on the order of seconds to hours for solids. In addition, the magnetisation in the xy plane will "dephase" through spin-spin or transverse relaxation processes, described by the time constant, $\mathrm{T}_{2}$. For solids, the apparent transverse relaxation (often termed $\mathrm{T}_{2}{ }^{*}$ )[15] is generally much faster than the longitudinal relaxation, and is typically on the ms timescale. 
The precession of $\mathbf{M}$ in the xy plane is recorded as the complex time-domain signal, $\mathrm{S}(\mathrm{t})$, or free induction decay (FID), as shown in Figure 2c, where the acquisition of both real and imaginary components (or "quadrature detection") by two detector coils allows the sense of precession to be determined. Assuming $T_{1} \gg T_{2}$, the FID can be written as

$$
S(t)=e^{i \Omega t} e^{-t / T_{2}}
$$

and Fourier transform yields the frequency-domain spectrum, $S(\omega)$, the real component of which is an absorptive Lorentzian function centred at $\Omega$ and with a full width at half height of $1 / \pi T_{2}$, as shown in Figure 2. As stated above, NMR spectroscopy is inherently insensitive and, consequently, the signal in the FID is generally accompanied by a large amount of electronic noise. This can be overcome by "signal averaging", where N FIDs are coadded with a "recycle interval" between successive acquisitions to allow magnetisation to return to equilibrium. As the signal increases linearly with $\mathrm{N}$, whereas the random noise accumulates with $\sqrt{ } \mathrm{N}$, the signal-to-noise ratio (SNR) increases as $\sqrt{ } \mathrm{N}$. The return of the $\mathrm{z}$ component of $\mathbf{M}, \mathrm{M}_{\mathrm{z}}$ to thermal equilibrium is given by

$$
\mathrm{M}_{\mathrm{z}}(\mathrm{t})=\mathrm{M}_{0}\left(1-\mathrm{e}^{-\mathrm{t} / \mathrm{T}_{1}}\right)
$$

meaning that equilibrium $\left(\mathrm{M}_{\mathrm{z}}(\mathrm{t})>0.99 \mathrm{M}_{0}\right)$ is only achieved at $\mathrm{t} \approx 5 \mathrm{~T}_{1}$. As a consequence, NMR experiments can be very time consuming for nuclei with low sensitivity or slow longitudinal relaxation.

\subsection{Internal Interactions}

Much of the practical value of NMR spectroscopy comes from the fact that nuclear magnetic moments interact not just with the external magnetic field, but also with their local surroundings (i.e., other nuclear spins, electrons and electric field gradients).[2-4] These interactions alter the resonant frequency of the nucleus (generally by a few $\mathrm{Hz}$ to tens of $\mathrm{kHz}$, compared to the 10-800 MHz Larmor frequency), making NMR spectroscopy an exquisitely sensitive probe of not just which nuclei are present in the material, but where exactly they are with respect to their surroundings. One of these interactions, the chemical shielding, arises from the motion of electrons in orbitals near the nucleus.[16] This motion creates a small magnetic field, $\mathbf{B}^{\prime}$, which always opposes $\mathbf{B}_{0}$ in a free atom, but can oppose or augment $\mathbf{B}_{0}$ in molecules and solids. The field at the nucleus is, therefore, given by 


$$
\mathrm{B}=\mathrm{B}_{0}-\mathrm{B}^{\prime}=\mathrm{B}_{0}(1-\sigma),
$$

where $\sigma$ is the field-independent shielding constant. It is practically challenging to measure the absolute value of $\sigma$ and, instead, the chemical shift, $\delta$, of an observed frequency, $\omega_{\mathrm{obs}}$ is generally quoted relative to a reference, $\omega_{\text {ref }}$ with

$$
\delta=10^{6} \times\left(\omega_{\text {obs }}-\omega_{\text {ref }}\right) / \omega_{\text {ref }},
$$

where the factor of $10^{6}$ accounts for the fact that $\delta$ is normally quoted as parts per million (ppm). As the sign of $\delta$ is opposite to that of $\sigma, \delta$ describes a deshielding scale. In solution, rapid molecular tumbling means that an average, or isotropic, chemical shift, $\delta_{\text {isor }}$ is observed, whereas such motion is absent for (most) solids and $\delta$ is anisotropic, described by a second-rank tensor, $\boldsymbol{\delta}$. In the principal axis system (PAS), $\boldsymbol{\delta}^{\mathrm{PAS}}$ is diagonal, with principal components $\delta_{11}, \delta_{22}$ and $\delta_{33}$, with $\left|\delta_{11}-\delta_{\text {iso }}\right| \geq\left|\delta_{33}-\delta_{\text {iso }}\right| \geq\left|\delta_{22}-\delta_{\text {iso }}\right|$ in the Haeberlen notation[17] (other notations are also commonly used - see Ref. [17] for details). The isotropic shift is given by

$$
\delta_{\text {iso }}=\operatorname{Tr}\{\boldsymbol{\delta}\}=\left(\delta_{11}+\delta_{22}+\delta_{33}\right) / 3,
$$

the anisotropy of the tensor, $\Delta_{\mathrm{CS}}$, is defined as

$$
\Delta_{\mathrm{CS}}=\delta_{33}-\delta_{\text {iso }},
$$

and the asymmetry by

$$
\eta_{\mathrm{CS}}=\left(\delta_{22}-\delta_{11}\right) / \Delta_{\mathrm{CS}},
$$

such that $0 \leq \eta_{\mathrm{CS}} \leq 1$. The observed chemical shift is given by

$$
\delta=\delta_{\text {iso }}+\left(\Delta_{\mathrm{CS}} / 2\right)\left[\left(3 \cos ^{2} \theta-1\right)+\eta_{\mathrm{CS}}\left(\sin ^{2} \theta \cos 2 \phi\right)\right],
$$

where the polar angles, $\theta$ and $\phi$, describe the orientation of $\delta$ relative to the laboratory frame. This chemical shift anisotropy (CSA) gives an orientation-dependent resonance for a single crystallite, and a characteristic powder-pattern lineshape, whose width depends on $\Delta_{\mathrm{CS}}$ and whose shape depends on $\eta_{\mathrm{CS}}$ as shown in Figure 3a. In an alternative formalism,[17] the anisotropy can also be described in terms of the span

$$
\Omega=\delta_{11}-\delta_{33},
$$

a measure of the magnitude, and the skew

$$
\kappa=3\left(\delta_{22}-\delta_{\text {iso }}\right) / \Omega,
$$

such that $-1 \leq \kappa \leq+1$. 
Nuclear spins can also interact with each other, either via the through-space dipolar coupling, or the electron-mediated J (or "scalar") coupling.[18] In the PAS, the dipolar interaction tensor, $\mathbf{D}^{\mathrm{PAS}}$, is traceless (i.e., there is no "isotropic dipolar shift") and axially symmetric $\left(\eta_{D}=0\right)$. The dipolar coupling between two spins, I and $S$, separated by a distance, $\mathrm{r}_{\mathrm{IS}}$, gives rise to a splitting of

$$
\omega_{\mathrm{D}}=\frac{\mu_{0}}{4 \pi} \frac{\mathrm{h} \gamma_{\mathrm{I}} \gamma_{\mathrm{S}}}{\mathrm{r}_{\mathrm{IS}}^{3}} \frac{1}{2}\left(3 \cos ^{2} \theta_{\mathrm{IS}}-1\right)
$$

where $\theta_{\mathrm{IS}}$ is the angle between the internuclear vector and $\mathrm{B}_{0}$. For a given $\mathrm{I} / \mathrm{S}$ spin pair, the only variable in Equation 19 is $\mathrm{r}_{\mathrm{IS}}$, making the dipolar coupling, in principle, a very sensitive measure of internuclear distances in solids. For isolated $(I=S=1 / 2)$ spin pairs in a powdered sample, the dipolar coupling gives rise to a "Pake doublet", as shown in Figure $3 b$. Such cases are rare and in reality a nucleus will generally experience multiple dipolar couplings of different magnitudes, leading to a more Gaussian-like lineshape, shown in Figure 3c.

The scalar coupling occurs as nuclear spins can polarise nearby electrons (typically in bonds), which then transfer this polarisation to other nearby nuclei. In solution, the scalar coupling leads to an isotropic splitting characteristic of the number and spin quantum number of the coupled nuclei, as shown in Figures 3d and 3e. The magnitude of the scalar coupling constant, J, typically decreases with the number of bonds through which the coupling occurs, in principle allowing the scalar coupling interaction to be used to probe through-bond connectivity. However, as J rarely exceeds a few hundred $\mathrm{Hz}$, the effects of the scalar coupling are often masked by other internal interactions in solids, and coupling patterns are rarely resolved. While $\mathrm{J}$ is technically a tensor quantity, giving rise to an anisotropic broadening as well as an isotropic splitting, only the isotropic splitting is generally considered as the anisotropy is sufficiently small as to be negligible under most common experimental conditions (as discussed below).

In addition to the interactions discussed above, nuclei with $\mathrm{I}>1 / 2$ possess a nuclear electric quadrupole moment, $\mathrm{eQ}$, which interacts with an electric field gradient 
(EFG), described by the tensor, V.[6-8,19] In its PAS, $\mathbf{V}^{\mathrm{PAS}}$ is diagonal and traceless, with components $\left|V_{z z}\right| \geq\left|V_{y y}\right| \geq\left|V_{x x}\right|$. The interaction of eQ with $\mathbf{V}$ is described by its magnitude, the quadrupolar coupling constant,

$$
\mathrm{C}_{\mathrm{Q}}=\mathrm{e} \mathrm{QV} \mathrm{zz} / \mathrm{h}
$$

and asymmetry,

$$
\eta_{\mathrm{Q}}=\left(\mathrm{V}_{\mathrm{xx}}-\mathrm{V}_{\mathrm{yy}}\right) / \mathrm{V}_{\mathrm{zz}}
$$

with $0 \leq \eta_{\mathrm{Q}} \leq 1$. The quadrupolar product, $\mathrm{P}_{\mathrm{Q}}$, can also be defined as

$$
\mathrm{P}_{\mathrm{Q}}=\mathrm{C}_{\mathrm{Q}}\left(1+\left(\eta_{\mathrm{Q}}^{2} / 3\right)\right)^{1 / 2}
$$

giving $\mathrm{C}_{\mathrm{Q}} \leq \mathrm{P}_{\mathrm{Q}} \leq 1.155 \mathrm{C}_{\mathrm{Q}}$. When the $\mathrm{EFG}$ at the nucleus is zero, all single-quantum $\left(\Delta \mathrm{m}_{\mathrm{I}}=\right.$ \pm 1 ) transitions occur at $\omega_{0}$. The effect of a small EFG can be considered as a first-order perturbation of the energy levels (shown for the case of $I=3 / 2$ in Figure 4a), with

$$
\omega_{\mathrm{Q}}=\left(\omega_{\mathrm{Q}}{ }^{\mathrm{PAS}} / 2\right)\left(3 \cos ^{2} \beta-1+\eta_{\mathrm{Q}} \sin ^{2} \beta \cos 2 \gamma\right),
$$

where $\beta$ and $\gamma$ are two of the Euler angles relating the PAS to the lab frame and (in rad s${ }^{-1}$ )

$$
\omega_{\mathrm{Q}}{ }^{\mathrm{PAS}}=3 \pi \mathrm{C}_{\mathrm{Q}} / 2 \mathrm{I}(2 \mathrm{I}-1) \text {. }
$$

For half-integer spins, the first-order perturbation leads to a differentiation between the central transition $\left(\mathrm{CT}, \mathrm{m}_{\mathrm{I}}=+1 \leftrightarrow \mathrm{m}_{\mathrm{I}}=-1\right)$ and the other single-quantum "satellite" transitions (STs) and, for a powdered sample, the CT occurs as a sharp line at $\omega_{0}$ (i.e., unaffected by the quadrupolar interaction), whereas the STs are broadened into Pake doublets, as shown in Figure $4 \mathrm{~b}$. Although they cannot be observed directly, it should be noted that the symmetrical multiple-quantum (MQ) transitions $\left(\Delta \mathrm{m}_{\mathrm{I}}=+\mathrm{n} \leftrightarrow \Delta \mathrm{m}_{\mathrm{I}}=-\mathrm{n}\right)$ are also unaffected by the first-order perturbation. The influence of a larger EFG can be considered as a second-order perturbation of the Zeeman energy levels, as discussed further in Refs. $[6-8,19]$. The second-order perturbation affects all transitions, with the STs broadened typically over several $\mathrm{MHz}$ and the CT shifted from $\omega_{0}$ by an isotropic quadrupolar shift, $\delta_{\mathrm{Q}}$, and broadened, typically by 1-10 kHz. In such cases, only the CT is observed, with its width proportional to $\mathrm{C}_{\mathrm{Q}}$ and a characteristic shape dependent on $\eta_{\mathrm{Q}}$, as shown in Figure 4c. The magnitude of the second-order quadrupolar interaction is proportional to $\left(\omega_{\mathrm{Q}}{ }^{2} / \omega_{0}\right)$, and so decreases with increasing $\mathrm{B}_{0}$ field. For nuclei with integer spin quantum number, there is no CT and all single-quantum transitions are anisotropically broadened by the first-order quadrupolar interaction, as shown in Figure 
$4 \mathrm{~d}$ for $\mathrm{I}=1$. However, the symmetrical multiple-quantum transitions $\left(\Delta \mathrm{m}_{\mathrm{I}}=+\mathrm{n} \leftrightarrow \Delta \mathrm{m}_{\mathrm{I}}=-\right.$ n) are unaffected to first order.

In addition to all of the interactions described above, when unpaired electrons are present, these may also affect the isotropic shifts and anisotropic broadening of resonances.[20,21] As electrons have spin $I=1 / 2$ and a gyromagnetic ratio $\sim 660$ times greater than ${ }^{1} \mathrm{H}$, through-bond and through-space interactions with unpaired electrons can be large and long range. The through-bond interaction is mechanistically analogous to the scalar coupling, but termed the hyperfine/transferred hyperfine interaction, with a coupling constant, $\mathrm{A} / \mathrm{h}$, usually on the order of $\mathrm{MHz}$. However, as the electronic relaxation is many orders of magnitude more rapid than nuclear relaxation, the nuclei experience only a thermally-averaged electronic spin state (rather than discrete $\alpha$ and $\beta$ spins), leading to a temperature-dependent isotropic shift rather than a splitting. The through-space pseudocontact interaction is mechanistically similar to the internuclear dipolar interaction, except that the electron spins are again thermally averaged, leading to a small isotropic shift in addition to the anisotropic broadening. Where present, the hyperfine coupling tends to dominate (with shifts of many hundreds or thousands of ppm possible) but both interactions are generally described by a single "paramagnetic shift anisotropy" (PSA) tensor, analogous to the CSA.[21] The rapid electronic relaxation can also induce very rapid nuclear $T_{1}$ and $T_{2}$ relaxation, often several orders of magnitude faster than in analogous diamagnetic materials. However, the precise effects of the unpaired electrons will depend strongly on the types of bonding present and the distribution of the paramagnetic centres within a material.

From the above discussion, it can be seen that an NMR spectrum of a material will contain a wealth of information: the number of resonances corresponds to the number of magnetically-distinct species (arising from chemical or crystallographic inequivalence), peak integrals should correspond to site populations, the anisotropic broadening contains information on point symmetry, the dipolar coupling can (in principle) be used to measure distances and the scalar coupling can provide valuable information about the bonding 
network present.[2-4] However, in general, all of this information is present simultaneously and, in most cases, the isotropic part of an interaction is much smaller than the anisotropic part. Therefore, when multiple resonances are present, their width tends to exceed their separation, leading to broadened and overlapped lineshapes that can often be very challenging to interpret, even for perfectly crystalline and ordered materials.

\section{Experimental Methodology}

From Section 2, it is clear that NMR spectra of solids suffer from two inherent problems low resolution and sensitivity - and many experimental approaches have been developed to overcome these. The resolution obtained from solution-state NMR spectroscopy is much greater than for solids, as the rapid isotropic molecular tumbling present leads to an effective averaging of the anisotropic interactions to their isotropic values. Such motion is not generally present in solids, but can be mimicked using the technique of magic angle spinning (MAS),[5] shown schematically in Figure 5a. In a MAS experiment, the sample is packed into a holder, or rotor, oriented at the "magic" angle of $\theta_{\mathrm{m}}=54.736^{\circ}$ to the $\mathrm{z}$ axis, and then rapidly rotated. If the rotation is sufficiently rapid (rotation rates up to at up to $\sim 111 \mathrm{kHz}$ are available using commercial hardware), MAS achieves the same average orientation $\left(\theta=54.736^{\circ}\right)$ for every crystallite, such that terms with an orientation dependence of $(1 / 2)\left(3 \cos ^{2} \theta-1\right)$, i.e., the chemical shift, dipolar and J couplings and the first-order quadrupolar interaction, are averaged to their isotropic values ( 0 for the dipolar and quadrupolar couplings, $\delta_{\text {iso }}$ for the chemical shift and J for the J coupling), as seen in Figure 5b.[2-4] If the MAS rate, $\omega_{R}$, is "slow" compared to the magnitude of the interaction to be removed, the powder-pattern lineshape is broken into a series of spinning sidebands (as seen in Figure 5b), separated by integer multiples of $\omega_{R}$, with intensities related to the static lineshape.[22] MAS is routinely used to enhance resolution, and has the added advantage that the spectral intensity is focused into narrow isotropic resonances (and sidebands), also aiding sensitivity. 
For quadrupolar nuclei, MAS is capable (in principle) of removing the first-order anisotropic quadrupolar broadening, although the magnitude of this interaction typically means that the ST are observed as a series of sharp spinning sidebands spanning hundreds of $\mathrm{kHz} \cdot[2,3,6]$ However, MAS while narrowing the lineshape is unable to completely remove the second-order anisotropic quadrupolar broadening of the CT, owing to its more complex angular dependence. It is not possible to remove the secondorder quadrupolar broadening by spinning around a single angle, but it is possible to achieve this by spinning at two angles simultaneously, as performed in the double rotation (DOR) approach.[2,23] In DOR, a sample is rotated at an angle of $30.56^{\circ}$ in a rotor that is held within a second rotor, inclined at $54.74^{\circ}$, using a specialist probe. However, the technical challenges of achieving stable spinning at two angles, and the diameter of the large outer rotor restrict the maximum rotation rate of the inner rotor to $\sim 2 \mathrm{kHz}$ and DOR spectra are often cluttered by spinning sidebands. Alternative methods, combining multiple-pulse sequences and standard probe hardware to achieve high resolution, are discussed below.

Heteronuclear spin-spin interactions (i.e., dipolar and scalar couplings) can be removed by decoupling, $[2,3,24]$ the simplest form of which involves observing the FID for one spin, I, while continuously irradiating the coupled spin, S. Such continuous-wave (CW) decoupling induces continuous transitions of the $S$ spins, such that the average coupling is zero. However, when the MAS rate is rapid, or the S spins experience large anisotropic interactions (e.g., CSA or quadrupolar), more efficient decoupling sequences may be required, and many of these have been developed (with a greater or lesser degree of transferability between different systems). Decoupling of homonuclear couplings $[2,3,25]$ is particularly challenging, as it involves the simultaneous irradiation and observation of the same spin. This is usually achieved by a complex "windowed" sequence, in which multiple pulses are applied to periodically refocus the homonuclear interactions to zero, with a data point recorded each time this occurs. Combining this with MAS (combined rotation and multiple pulse spectroscopy, or CRAMPS)[26] also requires 
many of the timings in the sequence to be synchronised with the sample rotation, imposing stringent requirements for experimental implementation.

In many cases, once a high-resolution spectrum has been achieved, it is desirable to reintroduce an interaction in a selective and controlled manner, such that its effect can be observed in the spectrum or it can be measured indirectly. A range of experiments have been developed to reintroduce the dipolar interaction (for the measurement of direct measurement of interatomic distances) and the CSA.[2,3,27-30] Information can also be obtained by transferring magnetisation between nuclear spins, typically measured using two-dimensional spectra.[31,32] A general two-dimensional experiment comprises four parts: preparation, evolution, mixing and detection. Preparation involves excitation of the first spin (or spins), via a pulse or series of pulses, with the magnetisation then allowed to evolve in a subsequent period of time (or time intervals and pulses), termed $t_{1}$. In the mixing stage, magnetisation is transferred between either heteronuclear or homonuclear spins. Finally, in the detection period, $\mathrm{t}_{2}$, the signal (i.e., FID) is recorded. The intensity of the signal recorded in $t_{2}$ is modulated by any evolution of the magnetisation in $t_{1}$ and, therefore, by recording a series of one-dimensional FIDs for systematically increasing values of $t_{1}$, it is possible to construct a two-dimensional dataset with "direct" $\left(t_{2}\right)$ and "indirect" $\left(t_{1}\right)$ dimensions. For nuclei with $I=1 / 2$, two-dimensional experiments are most commonly used to transfer magnetisation between nuclei (generally termed "correlation experiments") to determine the spatial (if the transfer is via the dipolar coupling) or covalent (if the transfer is via the scalar coupling) connectivity of a material. Twodimensional experiments can also be used to reintroduce interactions such as the CSA or scalar coupling in the indirect dimension (see above), allowing their accurate measurement where this is not possible from the MAS spectrum.[27, 29, 33]

For quadrupolar nuclei, the most popular two-dimensional experiment by far is the multiple-quantum (MQ) MAS experiment,[6,34] in which (after appropriate processing) the indirect dimension contains an isotropic spectrum with no contribution from the firstor second-order anisotropic quadrupolar broadening (i.e., a high-resolution spectrum) and 
the direct dimension contains MAS lineshapes from which the quadrupolar interaction parameters can be determined, as shown in Figure 5c. The experiment does not involve the transfer of magnetisation between spins, as discussed above, but rather the correlation of different transitions within the spin system, with the aim of removing completely the second-order quadrupolar interaction. However, MQMAS suffers from poor sensitivity, owing to the use of a formally "forbidden" multiple-quantum transition. While much effort has been devoted to developing pulse schemes[35-37] to improve the sensitivity of the experiment, the related satellite transition (ST) MAS experiment[6,38,39] offers much higher inherent sensitivity (owing to the use of only allowed single-quantum transitions throughout the experiment), albeit at the cost of a more exacting experimental setup in terms of MAS stability and the precision of the magic angle setting. Both experiments are, however, typically preferred to the one-dimensional DOR technique discussed above, as there is no need for specialist hardware. The vast majority of the two-dimensional magnetisation transfer experiments used for $I=1 / 2$ nuclei can also be applied to quadrupolar nuclei.[2,6,27] This will result in a broadened lineshape for the quadrupolar nucleus unless an initial MQ filtration step is applied in order to achieve high resolution.

In addition to issues with resolution, another major challenge facing solid-state NMR experiments is the inherently poor sensitivity. This arises not only from the low Boltzmann population differences at or near room temperature, but also from either the dilution of NMR-active isotopes (e.g., the only NMR-active isotopes of the two ubiquitous elements, $\mathrm{C}$ and $\mathrm{O},{ }^{13} \mathrm{C}$ and ${ }^{17} \mathrm{O}$ have natural abundances of just $1.07 \%$ and $0.037 \%$, respectively). It is possible to enhance the spin polarisation either by transfer from a nearby nucleus with higher polarisation (and, typically, higher abundance) such as ${ }^{1} \mathrm{H}$ or ${ }^{19} \mathrm{~F}$ using the cross polarisation (CP) experiment.[40,41] In a CP experiment, an initial $90^{\circ}$ pulse creates transverse magnetisation on the I spins, and this is transferred to the S spins (through the dipolar coupling between them) by simultaneous irradiation of both nuclei during a "spin lock" period. The rf field strength of the spin lock pulses must be "matched" such that $\omega_{1 \mathrm{I}}=\omega_{1 \mathrm{~S}}\left( \pm \mathrm{n} \omega_{\mathrm{R}}\right.$ in the case of MAS) in order to achieve efficient polarisation transfer. During spin lock, magnetisation is transferred at a rate described by 
the time constant $T_{\text {IS }}$ (dependent on the magnitude of the dipolar coupling) and decays by a process described by the time constant $T_{1 \rho}$. When $T_{1 \rho} \gg T_{I S}$, it is possible to achieve a theoretical polarisation enhancement of $\sim \gamma_{S} / \gamma_{\text {I }}$ (i.e., a factor of $\sim 4$ for ${ }^{1} \mathrm{H}$ and ${ }^{13} \mathrm{C}$ ). As polarisation is transferred via the dipolar interaction, $\mathrm{CP}$ is a non-quantitiative technique, with polarisation building up more rapidly for species such as $\mathrm{CH}_{3}$ than for, e.g., $\mathrm{CO}_{2}^{-}$. This non-quantitative nature of $\mathrm{CP}$ can be of use when assigning chemically-distinct species with similar chemical shifts. CP often provides no signal enhancement for quadrupolar nuclei, as it is difficult to achieve a transfer match for all crystallites present. However, the experiment can still be used for signal editing and spectral assignment, e.g., to distinguish between $\mathrm{SiOSi}$ and $\mathrm{SiOH}$ resonances. Another approach to improving sensitivity is the use of a Carr-Purcell-Meiboom-Gill (CPMG) echo train.[42,43] The application of a series of $180^{\circ}$ pulses during acquisition results in an FID that consists of multiple echoes, and subsequent Fourier transformation yields a spectrum consisting of a series of "spikelets", with intensities that reflect the lineshape, increasing the peak-height signal. The use of CPMG under MAS requires that the pulses are applied synchronously with the sample rotation.

The signal enhancement factor that is practically achievable when using other nuclei as the source of polarisation is generally of the order of a factor of 2-10 (although the typically more rapid relaxation of the I spins leads to a sensitivity enhancement per unit time that can be $c a$. two orders of magnitude). An alternative source of polarisation would be electrons, which have a much greater spin polarisation. The technique of dynamic nuclear polarisation (DNP) introduces unpaired electrons into a material in the form of (typically) nitroxide-based radicals.[44,45] At the magnetic fields used for NMR experiments, the electrons have a resonance frequency in the microwave region, and irradiation of the sample with microwaves followed by spin diffusion leads to a highlypolarised ${ }^{1} \mathrm{H}$ population. From here, the magnetisation can be transferred (e.g., by $\mathrm{CP}$ ) to the nucleus of interest, leading to a sensitivity enhancement factor of, in principle, up to $\gamma_{\mathrm{e}} / \gamma_{\mathrm{H}} \approx 660$, but, in practice, of 10-100. Although an extremely promising technique for improving sensitivity, DNP does require expensive hardware (e.g., a gyrotron to create 
suitable microwaves) and experiments to be performed at low (e.g., $100 \mathrm{~K})$ temperatures, posing a technical challenge and limiting the possible rotation rates. DNP is also surface sensitive and currently requires a sample to contain sufficient ${ }^{1} \mathrm{H}$ in close proximity to the species of interest. Applications to proteins, organic systems, polymers and catalyst surfaces have been demonstrated, $[44,45]$ but it is yet to be seen how routine this approach will become in the future for a wide range of systems.

In some cases, the resonance of interest is so broad that MAS is not a practical option (e.g., in paramagnetic samples or cases of very large values of $\Omega$ or $\mathrm{C}_{\mathrm{Q}}$ ) and it is more suitable to record the static lineshape.[6,46] Such "wideline" spectra are often recorded in a frequency-stepped fashion (i.e., in a series of sub spectra) in order to ensure uniform excitation at all frequencies, thereby avoiding any distortion of the lineshape. However, this can be time-consuming and practically challenging, and various broadband excitation pulses have been successfully applied (generally in conjunction with CPMG echo trains) to reduce the number of frequency steps required.

\section{The Effects of Disorder on NMR Spectra}

Any variation in the local environment of a nucleus will result in changes to the NMR parameters, i.e., in the isotropic and anisotropic shielding, the couplings between spins and the quadrupolar coupling. Unless dynamics are present (see below) the NMR spectrum of a disordered material can be seen as the sum of signals from every individual environment present. Hence, the appearance of the spectrum will depend on the type and extent of disorder present, and the magnitude of the changes in the NMR parameters.

If polymorphic disorder is present (i.e., the atoms and/or molecules adopt completely different spatial arrangements within a solid), the NMR spectrum may be significantly different in each case. This was shown in work on ${ }^{17} \mathrm{O}$ NMR spectra of four polymorphs of $\mathrm{MgSiO}_{3}$, [47] where each material had different numbers of distinct $\mathrm{O}$ species, all of which had very different quadrupolar and shielding parameters. In this case, 
the conditions required to synthesise each polymorph (i.e., the required temperature and pressure) are very different, and it is not likely they would be produced in the same sample. However, for many molecular and inorganic solids, this is not the case and there are a number of polymorphs with similar energies at room temperature and pressure, which can appear together in the same bulk sample. This is particularly true for small organic molecules and pharmaceuticals, and can pose a challenge for understanding a bulk material, if the structure solution is carried out by single-crystal diffraction (which, by definition, studies only a single polymorph per measurement). A similar problem can also be found for some inorganic materials, as shown in recent work on a range of niobate perovskites.[48-50] Peel et al.[50] showed that not only did samples of $\mathrm{Li}_{x} \mathrm{Na}_{1-x} \mathrm{NbO}_{3}(0.08 \leq$ $x \leq 0.20)$ contain mixtures of a polar orthorhombic phase $\left(P 2_{1} m a\right)$ and a rhombohedral phase $(R 3 c)$, the relative fractions of the two phases were shown to be critically dependent on synthetic conditions. Furthermore, the orthorhombic phase transformed slowly to the rhombohedral phase on standing in air at ambient temperature, a process that could be followed using ${ }^{23} \mathrm{Na}$ MQMAS NMR spectroscopy.[50]

If the variation in the local environment for an atom in a disordered material is significant (e.g., a change in coordination number), then this can produce a significant difference in chemical shift, and the appearance of separate resonances in the NMR spectrum. Less significant (or more remote) changes, in environment produce a smaller variation in the chemical shift, and the appearance of shoulders or splittings on the spectral lineshapes. When changes are much smaller (e.g., a variation in bond distances or angles), or are much more remote, the chemical shift change will be very small and typically leads to a spectral broadening. These effects can be seen on the ${ }^{89} \mathrm{Y}$ NMR spectra shown in Figure 6a. The ${ }^{89} \mathrm{Y}$ MAS spectrum of $\mathrm{Y}_{2} \mathrm{Sn}_{2} \mathrm{O}_{7}$ exhibits a single sharp resonance, confirming one type of $Y$ species is present, in a well-ordered solid, with the chemical shift confirming that the $\mathrm{Y}$ has eight-fold coordination.[51] The substitution of Ti onto the NNN sites in $\mathrm{Y}_{2} \mathrm{SnTiO}_{7}$ produces a number of shoulders and splittings of the lineshape, with the chemical shift varying with the number and arrangement of surrounding atoms.[51] There is also an increase in all of the linewidths in this spectrum, reflecting the long-range 
structural changes. The CPMG MAS spectrum of $\mathrm{Y}_{2} \mathrm{Zr}_{2} \mathrm{O}_{7}$ shows three very broad lineshapes, with the shift ranges confirming the presence of 6-, 7- and 8-coordinate $Y$, as a result of a disorder in the position of the oxygen vacancies in the structure.[52]

For systems where either only one geometrical parameter is varying, or where the variation is much more significant than that in any other parameter, it may be possible to relate the spectral broadening directly to the distribution of the geometrical parameters. This has been attempted in glasses by a number of authors, with the width of ${ }^{29} \mathrm{Si}$ NMR resonances being related directly to the distribution in the Si-O-Si bond angles.[53-55] However, such analyses are often complicated both by the fact that even in simple systems (e.g., $\mathrm{SiO}_{4}$ tetrahedra) more than one bond angle or distance needs to be considered. Furthermore, in most practical cases more than one geometrical parameter will vary simultaneously, complicating any quantitative spectral analysis. This was recently investigated for aluminophosphate (AlPO) frameworks, where the ${ }^{31} \mathrm{P}$ chemical shift was shown to depend not only on the average P-O-Al angle, as had been previously suggested, but also on the average P-O bond distance.[56] When performing two-dimensional experiments, the shape of the cross peaks observed, i.e., how the various contributions to each broad lineshape correlate with each other, can provide information on the spatial distribution of species in a sample. This can be seen in Figure $7 \mathrm{a}$, where a ${ }^{29} \mathrm{Si} /{ }^{29} \mathrm{Si}$ correlation spectrum of a surfactant-templated layered silicate is shown.[57] The elongated nature of the cross peaks at higher temperature reveals the presence of correlated disorder, with a Si species contributing to one part of one lineshape interacting (in this case through covalent bonds) to Si species that have only one chemical shift within the second resonance. More circular cross peaks, i.e., where all species that contribute to one lineshape interact with all that contribute to a second, would be observed if species were more randomly distributed.

It is also possible to obtain information on the nature of the order/disorder of surrounding species from the intensity of the spectral resonances. For example, if an atom is surrounded by four sites, each of which can be occupied by two different atomic species 
(X and $\mathrm{Y}$ ), there are five possible local environments (X4, X3Y, X2Y2, XY3 and Y4). Assuming these give rise to five resonances that can be resolved and accurately assigned, the relative intensity of each can be directly related to the amount of that environment present. Clearly, if clustering of $\mathrm{X}$ and $\mathrm{Y}$ is preferred, the intensity of the $\mathrm{X} 4$ and $\mathrm{Y} 4$ resonances will be greater than those from more mixed atomic distributions. Alternatively, if there is some preference for ordering, the X2Y2 resonance might have higher intensity. For a random distribution of the atoms on the surrounding sites, it is possible to predict the expected intensity of the resonances expected using simple statistics. For the general case, the probability of finding a particular environment, i.e., a atoms of species $\mathrm{A}, \mathrm{b}$ of species $\mathrm{B}, \mathrm{c}$ of species $\mathrm{C}$, etc., on $\mathrm{N}$ surrounding sites is given by

$$
\mathrm{P}=(\mathrm{N} ! / \mathrm{a} ! \mathrm{b} ! \mathrm{c} ! \ldots)(\mathrm{pA})^{\mathrm{a}}(\mathrm{pB})^{\mathrm{b}}(\mathrm{pC})^{\mathrm{c}} \ldots
$$

where $\mathrm{pA}, \mathrm{pB}$, etc., represent the probability of finding species $\mathrm{A}, \mathrm{B}$, etc., (which can be determined from the chemical formula), and $a+b+c \ldots$ has to equal N. For the specific case described above, this can be simplified to

$$
\left.\mathrm{P}=(\mathrm{N} ! / \mathrm{n} !(\mathrm{N}-\mathrm{n}) !)(\mathrm{pX})^{\mathrm{n}}(1-\mathrm{pX})\right)^{\mathrm{N}-\mathrm{n}},
$$

where $\mathrm{pX}$ represents the fraction of $\mathrm{X}$ and $1-\mathrm{pX}$ the fraction of $\mathrm{Y}$ in the sample. For example, for $\mathrm{pX}=0.6$ (i.e., there is $60 \% \mathrm{X}$ and $40 \% \mathrm{Y}$ distributed on the four sites), the probability of finding X4, X3Y, X2Y2, XY3 and Y4 environments would be 13.0\%, 34.5\%, $34.5 \%, 15.5 \%$ and $2.5 \%$, respectively. Therefore, if the relative spectral intensities are in agreement with this analysis then it can be assumed that the atomic distribution is random.

Although for spin $I=1 / 2$ nuclei it is often changes to the isotropic chemical shift that have the most significant effect upon the NMR spectrum, there are also changes to other NMR parameters as the local environment varies in a disordered solid. Changes in the isotropic shift result from a change in the principal components of the shielding tensor, which can result in a concomitant change in the anisotropic parameters $(\Omega$ and $\kappa)$. Therefore, it is often seen that the anisotropy varies across a spectral lineshape, i.e., with a change in $\delta_{\text {iso }}$. This can be measured using amplified PASS experiments, where the use of pulse trains allows the anisotropic interaction to be recoupled, or reintroduced, and 
measured in the indirect dimension of a two-dimensional experiment. This is shown in Figure 7b, where CSA measurements (specifically $\Omega=\delta_{33}-\delta_{11}$ ), acquired using amplified PASS experiments are shown overlaid on the ${ }^{89} \mathrm{Y}$ MAS NMR spectrum of $\mathrm{Y}_{2} \mathrm{Zr}_{2} \mathrm{O}_{7}$.[52] Three ${ }^{89} \mathrm{Y}$ signals are seen (corresponding to $\mathrm{Y}^{\mathrm{VI}}, \mathrm{Y}^{\mathrm{VII}}$ and $\mathrm{Y}^{\mathrm{VIII}}$ ) owing to the disorder of the oxygen vacancies on the anion lattice of this defect fluorite material. For $Y^{\mathrm{VIII}}, \Omega$ varies across the broadened lineshape, decreasing with decreasing $\delta_{\text {iso }}$. In contrast, there is little change in $\Omega$ across the $Y^{\mathrm{VII}}$ lineshape. The J coupling can also vary as the local environment changes. For a disordered material, where the distribution of chemical shift may well obscure any splittings resulting from J couplings, these can be measured in the indirect dimension of a two-dimensional J-resolved spectrum. As shown in Figure 7c for a ${ }^{29} \mathrm{Si}$ spectrum of $\left({ }^{29} \mathrm{Si}\right.$ enriched) $\mathrm{SiO}_{2}$, the changes in $\mathrm{J}$ can be correlated to those in the chemical shift, resulting in a J coupling that varies at different positions within the spectral lineshape.[58]

For nuclei with spin $I>1 / 2$, variation in the local structure changes not only the chemical shift, but also the magnitude and asymmetry of the quadrupolar interaction. As the second-order quadrupolar interaction produces resonances that have a characteristic shape (determined by $\eta_{\mathrm{Q}}$ ) disorder produces not just a broadening of the line, but also a change in its shape. This can be seen in the ${ }^{27} \mathrm{Al}$ MAS NMR spectrum of $\gamma-\mathrm{Al}_{2} \mathrm{O}_{3}$ shown in Figure $6 \mathrm{~b}$, where the resonances display a characteristic "tail" to low frequency resulting from the distribution of quadrupolar parameters.[6] The exact shape of the line is determined by the magnitude (and the type) of the distributions in $\delta_{\text {iso }}, \mathrm{C}_{\mathrm{Q}}$ and $\eta_{\mathrm{Q}}$, and whether these are correlated. It is difficult to extract this information from a single MAS lineshape unless some assumptions about the distributions can be made. One approach to simplify this problem is to assume a joint distribution in the quadrupolar parameters using the Czjzek model (and its various extensions),[59-61] exploiting the fact that both $C_{Q}$ and $\eta_{\mathrm{Q}}$ are related to the principal components of the EFG tensor. Thus, the distribution in the quadrupolar parameters can be described by a single parameter. Lineshapes can then be fitted assuming a Czjzek distribution of the EFG and, typically, a Gaussian distribution of $\delta_{\text {iso. }}$ Further insight into the distributions of parameters present can be obtained by 
considering the shape of the resonances in two-dimensional MQMAS spectra. As seen in Figure $6 \mathrm{~b}$ for $\gamma-\mathrm{Al}_{2} \mathrm{O}_{3}$, MQMAS lineshapes for disordered materials contain ridges that are broadened along different gradients by distributions in chemical shifts and/or the quadrupolar parameters. These axes are shown on the MQMAS spectrum in Figure 6b for a spin $I=5 / 2$ nucleus (note that, in the spectrum shown, an ordered material would exhibit a ridge-like lineshape that lies parallel to the $\delta_{2}$ axis, as shown in Figure 5c).[62] Using freely-available fitting programs,[63] it is possible to fit the two-dimensional lineshapes (typically assuming a Czjzek distribution of the EFG and a Gaussian distribution of the chemical shift) and extract the information on the magnitude of the distribution of the NMR parameters present.

In many materials the positions of atoms or groups vary over time, leading to temporal or dynamic disorder. It can be extremely challenging to distinguish this case from that where atomic positions vary in different parts of a material (i.e., static positional disorder), with diffraction providing information simply on the average structure, and refining sites as having a fractional occupancy. NMR spectroscopy, however, is sensitive to motion on timescales spanning 15 orders of magnitude, as shown in Figure 8.[2,3] Very fast motion leads to changes in the relaxation processes, with $T_{1}$ relaxation affected by dynamic processes with a correlation time $\tau_{\mathrm{c}} \approx 1 / v_{0}$. Slow motion can be measured using two-dimensional exchange experiments, where cross peaks show the change in the NMR parameters that result from the physical motion of an atom when a "mixing time" (usually $10^{-3}$ to $10^{2} \mathrm{~s}$ ) is introduced into the pulse sequence. Although resolution is higher in MASbased experiments (providing site specific information), in some cases information on the change in geometry (i.e., the type of motional process) is more easily obtained from the shape of the cross peaks in static experiments. In general, the position, shape and width of the resonances within an NMR spectrum are affected by motion on timescales intermediate to these two extremes, resulting from changes in the shielding, dipolar or quadrupolar couplings as atomic environments vary.[2,3,6] This could result from motion of an atom itself, or from changes in the position of surrounding atoms. In most cases, changes in the lineshapes and/or linewidths can be followed as a function of temperature, 
enabling information on the type of motion, its timescale and activation energy to be obtained. The most significant effects are observed when the dynamic process has a correlation time similar to the inverse of the magnitude of the anisotropic broadening. If experiments are performed under MAS, dynamics on the timescale of 10-100 $\mu$ s (i.e., $\approx$ $\left.1 / v_{R}\right)$ can also affect the desired averaging of sample rotation, resulting in a temperaturedependent broadening of the lineshape.[2,6,64,65] A similar broadening can affect the STMAS experiment (used to obtain high-resolution spectra for quadrupolar nuclei, as described above).[66] This provides site-specific information on dynamics, and the comparison between MQMAS and STMAS spectra can help to identify motional processes on the $\mu$ s timescale, as demonstrated by work on the dynamics of guest within phosphate materials, and ${ }^{1} \mathrm{H}$ motion in silicates.[65,66]

\section{Calculation of NMR Parameters}

The prediction of NMR parameters using quantum-chemical calculations has been possible for many years although, until relatively recently, these have been applied predominantly to discrete systems. $[67,68]$ While it is perhaps easy to see how a molecular solid might be considered in this approach, most periodic solids must be modelled as a discrete "molecule" or cluster of atoms, with any "hanging" bonds terminated, usually by hydrogen atoms, as shown schematically in Figure 9a. The most obvious benefit of treating a periodic solid in this way is that it reduces the problem to one of calculating the NMR parameters for a finite number of atoms, significantly reducing the cost associated with any calculation. While initially it may seem acceptable (and, in many cases, a necessity) to treat a repeating periodic solid as a cluster, this approach does have inherent limitations, and may not provide a sufficiently accurate model of the true solid-state structure. Terminating the cluster introduces a surface layer not present in a periodic solid, which can affect the electronic properties of the bulk, although this effect decreases exponentially as the cluster size increases. Additionally, the electric field, which is zero in periodic solids, is non-zero in all but the largest clusters,[68] possibly affecting the computed NMR parameters and certainly casting doubt on their accuracy. 
The introduction of the gauge-including projector augmented wave (GIPAW) approach by Pickard and Mauri in 2001 heralded a step change in the use of the theoretical calculation of NMR parameters for periodic solids.[12] GIPAW allows the chemical shielding for a periodic system to be calculated, using a planewave basis set (see later), exploiting the inherent translational symmetry of the solid state. This is achieved by recreating an infinitely repeating structure from a unit cell (Figure 9b), from which the NMR parameters for all atoms can be calculated simultaneously (i.e., there is no restriction that the calculation is only accurate for the atom at the centre of a hypothetical molecular cluster). This approach has gained widespread popularity, particularly among the experimental community, and has been applied to the study of microporous materials, pharmaceuticals, ceramics, minerals and glasses.[9,10,69] For ordered solids, calculations are primarily used to interpret and assign spectral resonances, and to provide supporting evidence for magnitude of parameters that are difficult to measure experimentally (e.g., anisotropic interactions or tensor orientations) and to suggest the presence of interactions or couplings between atoms. Calculations can provide insight into the effect of structural changes on NMR parameters, and can be used to predict spectra and guide experimental measurement, particularly when sensitivity is limiting. For disordered materials, in principle, calculations offer unique insight into the understanding (and decomposition) of the complex spectral lineshapes typically encountered, and enable the prediction of NMR parameters for a series of possible structural models. In addition, significant differences between experiment and calculation can suggest the presence of dynamics, and can be used to determine the average parameters that might be measured experimentally.

To compute NMR parameters requires an approximation of the time-independent non-relativistic Schrödinger equation, providing the total energy of the system. However, even with simplifications such as the Born-Oppenheimer approximation, solving the Schrödinger equation is often unfeasible for large systems. Therefore, the majority of calculations for solids utilise the density functional theory (DFT) framework, where the 
ground state electronic energy, $\mathrm{E}_{0}$, is defined as a functional solely of the electron density[70,71]

$$
\mathrm{E}_{0}[\rho(\mathrm{r})]=\mathrm{E}_{\mathrm{Ne}}[\rho(\mathrm{r})]+\mathrm{T}_{\mathrm{S}}[\rho(\mathrm{r})]+\mathrm{J}[\rho(\mathrm{r})]+\mathrm{E}_{\mathrm{XC}}[\rho(\mathrm{r})] .
$$

$\mathrm{E}_{\mathrm{Ne}}, \mathrm{T}_{\mathrm{S}}$ and $\mathrm{J}$ represent the attractive nucleus-electron interaction energy, kinetic energy of a non-interacting electron and the electron-electron Coulomb interactions, respectively. These three terms are known and can be calculated efficiently, whereas the form of the final term, $E_{\mathrm{XC}}$ (the exchange-correlation energy), is not known exactly and must be approximated. The simplest exchange-correlation functional, the local density approximation (LDA), $[67,68,72]$ assumes that the electron density is constant over small distances, allowing it to be approximated as that of a uniform electron gas of equivalent density. Though suitable for many systems, the LDA functional has a tendency to overbind,[67,68] resulting in overestimated atomization energies and underestimated interatomic distances. The generalized gradient approximation (GGA) provides an improvement on the LDA, as it includes the gradient of the electron density, $\nabla \rho(\mathrm{r})$, in the exchange-correlation functional.[73,74] Further accuracy improvements for calculating $\mathrm{E}_{\mathrm{XC}}$ can be achieved by employing hybrid-GGA or meta-GGA functionals,[75] although the cost of these approaches often restricts their use in the solid state.

Given the periodic nature of most solids, Bloch's theorem,

$$
\mathrm{V}(\mathbf{r}+\mathbf{L})=\mathrm{V}(\mathbf{r})
$$

applies, with $\mathrm{V}(\mathbf{r})$ defining the potential at $\mathbf{r}$ and $\mathrm{V}(\mathbf{r}+\mathbf{L})$ the potential at $\mathbf{r}$ displaced by the lattice vector $\mathbf{L}$, enabling the ground state electronic energy of a solid to be determined from its unit cell. As the electron density is also periodic, so too is the magnitude of the wavefunction $\Psi(\mathbf{r})$. However, the phase is only pseudoperiodic, giving

$$
\Psi(\mathbf{r})=\mathrm{e}^{\mathrm{ikr}} \mathrm{u}_{\mathrm{k}}(\mathbf{r}),
$$

where $\mathrm{e}^{\mathrm{ikr}}$ is a phase factor and $\mathbf{u}_{\mathrm{k}}(\mathbf{r})$ is a periodic function. In many DFT codes designed to calculate properties for solids, the wavefunction is represented using a planewave basis set, of the form

$$
\mathrm{u}_{\mathrm{k}}(\mathbf{r})=\Sigma_{\mathrm{G}} \mathrm{C}_{\mathrm{ck}} \mathrm{e}^{\mathrm{i}_{\mathrm{G}} \cdot \mathrm{r}},
$$


where $c_{\mathrm{c}_{\mathrm{k}}}$ are Fourier coefficients and G are reciprocal lattice vectors, often termed "Gvectors". The quality of a planewave DFT calculation is primarily determined by two factors: the number of indices $\mathrm{k}$ or "k-points" used to sample the first Brillouin zone in reciprocal space; and the number of G-vectors for the number of basis functions, which is described via a kinetic energy cut off.

The efficiency of calculations is improved in many codes by using pseudopotentials. $[9,12,68]$ These enable two approximations relating to the atomic core to be made. The first, termed the frozen core approximation, treats electrons within a defined atomic core radius $\left(\mathrm{r}_{\text {cut }}\right)$ as "frozen", and only the valence electrons are treated explicitly. In the second, the rapid oscillations in the wavefunction of the valence electrons close to the nuclei can be substituted by a "smoothed" or "pseudised" version, simplifying the description of this region when using planewaves, reducing the basis set size, and thus increasing calculation efficiency. Although these approximations provide significant cost benefits, with little loss in accuracy (as the total energy and many chemical properties rely primarily on the valence electrons), some properties (importantly including the nuclear shielding) require a full treatment of the electrons close to the nucleus. The all-electron wavefunction can be reconstructed using the projector augmented wave (PAW) approach,[76] with the GIPAW[12] extension required to compute magnetic response properties.

An appropriate and accurate structural model is a vital pre-requisite for the prediction of NMR parameters. In many cases, these are generated from diffraction experiments (although models from prior computational work or from other experimental measurements can also be employed).[11] The errors inherent in experimental structure solution, particularly when using X-ray diffraction, where the position of light atoms is often not determined directly and isoelectronic atoms cannot be distinguished, often necessitates the optimization of the geometry prior to the calculation of NMR parameters.[77] Such optimizations can involve varying only the position of the light atoms, varying all atomic co-ordinates and/or varying the unit cell parameters. Any 
optimisation does require some caution, however, as the neglect of dispersion interactions by some of the functionals used in DFT can produce inaccurate results, with overestimated interatomic distance or cell dimensions. $[9,11,78]$ This can be a particular problem in molecular crystals, or in flexible inorganic solids such as phosphate frameworks or MOFs. $[9,11,78]$ Recently, this has been overcome in many codes with the introduction of semi-empirical dispersion correction (SEDC) schemes,[11,79] which attempt to account for the attractive term in the "12-6" Lennard-Jones potential.

For ordered solids, the periodic approach described above is both efficient and intuitive. However, for disordered materials, where this periodicity is not rigidly retained, periodic boundary conditions limit the possible environments that can be considered computationally. It might not be possible to generate models that correspond exactly to the "real" structure, and simplified approaches, aimed at understanding the relative variations in the NMR parameters, rather than determining their exact magnitude, have to be employed. The use of a cluster model, discussed above, can provide insight into the variation of NMR parameters with structural changes, and this may be sufficient to assign resonances or explain the appearance of a spectral lineshape, even if an exact magnitude is not accurately calculated. For the case of low-level compositional disorder, insight can be gained simply by substituting one atom into the unit cell of a periodically-ordered counterpart, providing information on the type and magnitude of the changes expected for the surrounding atoms and predicting NMR parameters for the substituted species. Examples of recent studies include work on Mg-substituted hydroxyapatite, Alsubstituted magnesium silicates, Mg-substituted AlPOs, Y substitution into perovskites, $\mathrm{Sn} / \mathrm{Ti}$ disorder in pyrochlore ceramics and $\mathrm{F}$ substitution into hydrous minerals. $[9,11,69,80-86]$ A better approximation for an aperiodic structure (particularly for low level disorder) can be obtained by using a repeating crystal composed of "supercells", as shown in Figure 9c, where the supercell is chosen to be sufficiently large to minimise the effect of any structural change on the neighbouring cells. It should also be noted that using a supercell increases the number of atoms to be treated explicitly, thereby increasing the computational cost. Higher levels of substitution pose a more significant combinatorial 
challenge, with many different arrangements of atoms possible (either in a single cell or a supercell). Two different approaches can then be envisaged. The first involves the consideration of a limited number of possible structural models, typically constructed by varying the local environment of one atom within the system systematically, effectively modelling disorder as a cluster-like defect within a periodic three-dimensional structure.[11,52,82] A more sophisticated approach is to consider a much greater set of possible atomic arrangements, either generated completely randomly (ensuring for efficiency reasons that symmetrically-related structures are not considered multiple times), or ranked in some way (e.g., by enthalpy) to restrict the subsequent computational cost of NMR calculations. It should be noted, however, that for all structures that have been adapted or generated computationally, full geometry optimisation will be necessary, as the specific arrangement of atoms with the computational unit cell no longer matches any average structure produced by diffraction.[9]

For the case of positional disorder it may be feasible to consider all possible atomic arrangements if the number of possible sites a species or group can occupy is relatively low, as demonstrated recently for materials including AlPO frameworks, dense phase ceramics and layered silicates. Similarly, disorder in the stacking of molecules or layered materials can often be studied using a limited number of possible structural models. $[9,11,87,88]$ When atoms or groups are placed manually, only a limited subset of models is typically considered, and there may also be some bias introduced in the types of positions chosen. In contrast, automated computational approaches can generate large sets of candidate structures, often with the reduction of bias. For example, the study of disorder in glasses has been approached recently by combining molecular dynamics (MD) calculations with NMR spectroscopy.[89,90] The use of classical MD enables larger systems to be studied, ensuring periodicity is not imposed on too small a length scale. Any issues with transferability of the empirical forcefields used can be overcome with ab initio $\mathrm{MD}$, although this does limit the number of atoms and the dynamic timescales that can be investigated. Alternative approaches for generating structural models when less detailed information is available include structure prediction methods. These computational 
approaches can generate candidate crystal structures when given a molecular formula or information on symmetry and stoichiometry. Many approaches have been used to help solve or refine structures and to probe polymorphic disorder.[9,11] More recently, the $a b$ initio random structure searching, or AIRSS, $[91,92]$ method has been applied to investigate disordered solids. This approach produces structures from randomised cells and random atomic coordinates and optimised directly using DFT. Although the use of DFT brings increased cost, it does facilitate the combination of AIRSS with the calculation of NMR parameters, and direct comparison to experimental NMR spectra. This was demonstrated in recent work using AIRSS to investigate the hydration of wadsleyite, a high-pressure silicate mineral.[93] Although the structure of the anhydrous mineral $\left(\beta-\mathrm{Mg}_{2} \mathrm{SiO}_{4}\right)$ is known, the position of $\mathrm{H}$ in the hydrated form could not be determined from diffraction. As shown schematically in Figure 10a, starting from the structure of anhydrous $\beta-\mathrm{Mg}_{2} \mathrm{SiO}_{4}$, a $\mathrm{Mg}$ vacancy was created, two $\mathrm{H}$ randomly placed according to the AIRSS protocol, and the geometry optimised. After repeating this procedure, the resulting structures were ranked according to energy. As shown in Figure 10b, lowest energy structures all had $\mathrm{H}$ placed on $\mathrm{O} 1$ atoms, with protonation of the silicate oxygens only in higher-energy structures. Calculated ${ }^{1} \mathrm{H}$ NMR parameters (Figure 10c) are shown to be strongly dependent on the $\mathrm{O}-\mathrm{H}$ and $\mathrm{H}$... O hydrogen bond distances, enabling these to be determined directly from the spectrum for each resonance. Calculations were also able to assign the spectral resonances, with experimental spectra suggesting $\sim 85 \%$ of protonation occurred at 01 , and $~ 15 \%$ at the silicate oxygens.[93] 


\section{Application of NMR Spectroscopy to Disordered Materials}

In this section we will illustrate the use of solid-state NMR spectroscopy to investigate disordered solids. We have chosen to divide the discussion by the type of materials considered, although many of the points highlighted will clearly be relevant to more than one system. This cannot be a comprehensive discussion, and so readers will be referred to existing review articles for more information on each type of material discussed.

\subsection{Ceramics and Oxides}

An oxide is a chemical compound that contains at least one oxygen anion (usually in the 2 oxidation state). In metal oxides, this charge is balanced by at least one cationic metal species, giving binary (e.g., $\mathrm{AO}, \mathrm{AO}_{2}, \mathrm{~A}_{2} \mathrm{O}_{3} \ldots$ ), and ternary (e.g., $\mathrm{ABO}, \mathrm{ABO}_{3}, \mathrm{~A}_{2} \mathrm{BO}_{4}, \ldots$ ) etc... compounds. Many oxides are also classed as ceramics (formally inorganic nonmetallic solids containing at least one metal and one non metal). The crystallinity of ceramics varies from highly ordered to completely amorphous, and most display high melting temperature and hardness, poor conductivity and good chemical resistance, although there are known exceptions. A variety of structure types are adopted by oxides and ceramics, many of which are able to incorporate many different cations and cation combinations. This leads to a range of different physical and chemical properties and a variety of commercial, technological and industrial applications, including in semiconductors and electronics, energy materials, catalysis, coatings and paints and as wasteforms. The wide utility of oxides and ceramics makes it vital to understand the structure-property relationships in these materials. Chemically-flexible structures, such as perovskite or pyrochlore, tolerate many defects, substitutions and variable oxidation states, providing a significant challenge for structural characterization. The need for structural insight over different lengthscales (and, indeed, different timescales) is crucial, requiring a combination of information on the average structure (from diffraction-based approaches) and the local structure and ordering, typically from spectroscopy. For more information on NMR of oxides and ceramics see Refs. [4,6,94]. 
Compositional disorder (be it in the form of cation mixing, partial site occupancy or a variation in oxidation state) is common in oxides and ceramics, and has long been studied using solid-state NMR spectroscopy, with the chemical shift typically very sensitive to substitution of neighbouring and next-nearest neighbouring (NNN) atoms. In a comprehensive study of rare-earth pyrochlores, Grey et al. observed a number of unusual resonances in the ${ }^{89} \mathrm{Y}$ NMR spectra of $\mathrm{Y}_{2-\mathrm{y}} \mathrm{Ln}_{\mathrm{y}} \mathrm{M}_{2} \mathrm{O}_{7}(\mathrm{Ln}=\mathrm{Ce}, \mathrm{Pr}, \mathrm{Nd}, \mathrm{Sm}, \mathrm{Eu}, \mathrm{Yb}$; $\mathrm{M}=\mathrm{Sn}$, Ti) materials.[95] For the diamagnetic end members, only a single resonance was observed, corresponding to $\mathrm{Y}$ on the crystallographically-unique eight-coordinate A site of the pyrochlore structure. In many cases, substituting a small amount of $\mathrm{Ln}^{3+}$ (producing $\mathrm{Y}(\mathrm{OY})_{6-\mathrm{n}}(\mathrm{OLn})_{\mathrm{n}}$ environments) resulted in the observation of additional ${ }^{89} \mathrm{Y}$ resonances, often at unusual shifts (e.g., a change of -80 ppm for $\mathrm{Eu}^{3+}$ substitution, and +82 ppm for $\mathrm{Pr}^{3+}$ substitution), resulting from a paramagnetic interaction with the unpaired $\mathrm{f}$ electrons on NNN A-site cations. The shifts were found to be proportional to the number of $\operatorname{Ln}^{3+}$ ions on the NNN sites, and generally larger for the titanates than for the stannates. Although additional resonances were observed for $\mathrm{Ce}^{3+}, \mathrm{Pr}^{3+}, \mathrm{Nd}^{3+}, \mathrm{Eu}^{3+}$ and $\mathrm{Yb}^{3+}$ substitution, only one resonance was observed for materials with substitution of $\mathrm{Sm}^{3+}$, $\mathrm{Ho}^{3+}$ or $\mathrm{Tm}^{3+}$, either as a result of the very fast relaxation or the linebroadening induced by the unpaired electrons. The shifts exhibited a significant temperature dependence and were shown to have a significant pseudocontact contribution to the paramagnetic interaction. The intensity of the spectral resonances decreased as the number of $\mathrm{Ln}^{3+} \mathrm{NNN}$ increased, and no peaks were seen for $n>3$, suggesting only small amounts of cations were substituted. The composition of the materials could be estimated from the relative spectral intensities, which would be difficult to obtain with comparable precision from diffraction. The paramagnetic shifts observed were much smaller than those seen previously in ${ }^{119} \mathrm{Sn}$ NMR spectra of $\mathrm{Ln}_{2} \mathrm{Sn}_{2} \mathrm{O}_{7}$ (e.g., -3670 for $\operatorname{Pr}^{3+}$ and +5480 for $\mathrm{Eu}^{3+}$ ), despite NNN substitution in both cases. However, the more covalent character of the Sn-O bond was shown to result in a greater through-bond (or Fermi contact) contribution to the paramagnetic interaction. $[96,97]$ 
More recently, NNN substitution in diamagnetic $\mathrm{Y}_{2} \mathrm{Ti}_{2-\mathrm{x}} \mathrm{Sn}_{\mathrm{x}} \mathrm{O}_{7}$ pyrochlores was studied using a combination of ${ }^{89} \mathrm{Y}$ and ${ }^{119} \mathrm{Sn}$ NMR spectroscopy and DFT calculations.[51,82-84] Selected ${ }^{89} \mathrm{Y}$ and ${ }^{119} \mathrm{Sn}$ spectra in Figure 11a show only $\mathrm{Y}^{\mathrm{VIII}}$ and $\mathrm{Sn}{ }^{\mathrm{VI}}$ are present (i.e., $\mathrm{Y}$ and $\mathrm{Sn}$ sitting on the $\mathrm{A}$ and $\mathrm{B}$ sites of the pyrochlore structure, respectively, suggesting there is very little A/B mixing in these systems). This was also confirmed using DFT calculations, which predicted significantly different shifts for $\mathrm{Y}^{\mathrm{VI}}$ and $\mathrm{Sn}^{\mathrm{VIII}}$. The ${ }^{89} \mathrm{Y}$ NMR spectra show a range of resonances, suggesting different $\mathrm{Y}$ environments whereas, in contrast, ${ }^{119} \mathrm{Sn}$ spectra show additional resonances at low $\mathrm{Ti}$ content but a single broad resonance as the Ti content increases. Although occupying different sites in the structure, Figure 11a shows that $Y$ and Sn both have 6 NNN B sites, and so B-site substitution of $\mathrm{Sn} / \mathrm{Ti}$ might be expected to produce a similar change to the spectrum in each case. DFT calculations (Figure 11b) of ${ }^{89} \mathrm{Y}$ and ${ }^{119} \mathrm{Sn}$ chemical shifts for models where the number and arrangement of $\mathrm{Sn} / \mathrm{Ti}$ on the surrounding NNN sites were varied, revealed that although a systematic change in ${ }^{89} \mathrm{Y}$ chemical shifts was observed with Ti substitution, the change in the ${ }^{119} \mathrm{Sn}$ shift became much smaller with increasing numbers of $\mathrm{Ti} \mathrm{NNN}$, predicting the overlap of the spectral resonances (as seen experimentally). Considering calculated and experimental isotropic and anisotropic shielding enabled assignment of all spectral resonances, while their relative intensities revealed a random distribution of B-site cations (using Equation 26 above). Similarly, random distributions of $\mathrm{B}$-site cations in $\mathrm{Y}_{2} \mathrm{Zr}_{2-x} \mathrm{Sn}_{\mathrm{x}} \mathrm{O}_{7}$ pyrochlores and of $\mathrm{A}$ site cations in $\mathrm{Y}_{2-\mathrm{x}} \mathrm{La}_{\mathrm{x}} \mathrm{Ti}_{2} \mathrm{O}_{7}$ pyrochlores have also been shown using NMR spectroscopy, although a relatively limited solid solution was seen in both cases.[52,98]

For $\mathrm{Y}_{2} \mathrm{Zr}_{2-\mathrm{x}} \mathrm{Sn}_{\mathrm{x}} \mathrm{O}_{7}$ ceramics a pyrochlore phase is expected at high $\mathrm{Sn}$ content, but as the amount of $\mathrm{Zr}$ increases a defect fluorite phase is predicted, with complete disorder of the cation lattice and of the anions/vacancies (i.e., both compositional and positional disorder).[52,99] The transition between the two is predicted (using idealised radius ratio rules) to occur at $x=1.5$. As was seen in Figure $6 a$, the ${ }^{89} Y$ MAS NMR spectrum of $\mathrm{Y}_{2} \mathrm{Zr}_{2} \mathrm{O}_{7}$ contains very broad spectral resonances, reflecting the considerable disorder, with $\mathrm{Y}^{\mathrm{VI}}, \mathrm{Y}^{\mathrm{VII}}$ and $\mathrm{Y}^{\mathrm{VIII}}$ present, at $\sim 80, \sim 185$ and $\sim 290$ ppm, respectively, owing to the anion disorder. 
The relative intensities of the resonances $(5.6 \%, 64.9 \%$ and $29.4 \%)$ suggest an average $\mathrm{Y}$ coordination number of 7.2 (and, therefore, of 6.8 for $\mathrm{Zr}$ ), confirming the preference of the vacancies to associate with $\mathrm{Zr}$ in this material. Vacancy ordering was also observed for the Sn-substituted materials using NMR spectroscopy, with Sn shown to be exclusively six coordinate.[52,99] The coordination number of Zr was shown to decrease (from 6.8 to 6.5) with Sn substitution, while that of $\mathrm{Y}$ increased (from 7.2 to 8). Unlike the diffraction experiments, NMR spectroscopy showed a significant two-phase region in this series, i.e., where pyrochlore and defect fluorite phases were present simultaneously, between $\mathrm{x}=0.8$ and 1.6. Integration of the spectral resonances was able to provide information on the proportion of each phase present and their compositions.[52] A solid-solution limit of $\sim 13 \% \mathrm{Zr}$ into the pyrochlore was observed, whereas the composition of the defect fluorite phase varied throughout.

The well-known perovskite $\left(\mathrm{ABO}_{3}\right)$ structure, consisting (ideally) of corner-sharing $\mathrm{BO}_{6}$ with the A cation having 12-fold coordination, exhibits extremely high chemical flexibility, and is able to incorporate a vast array of different cations. This substitution can lead to a number of structural modifications, including cation displacements and tilting or distortion of the octahedra. The possible presence of polymorphic, compositional and positional disorder has resulted in many NMR investigations, with insight into the atomicscale structure and ordering the ultimate aim. One of the most studied pervoskites is $\mathrm{NaNbO}_{3}$, owing to reports of exceptional piezoelectric responses in ceramics based on $\mathrm{NaNbO}_{3}$ solid solutions, and suggestions of an extremely complicated, but poorly understood, phase diagram. Building on earlier NMR studies of $\mathrm{NaNbO}_{3},[100,101]$ Johnston et al. used ${ }^{23} \mathrm{Na}$ MQMAS experiments to show that many samples of $\mathrm{NaNbO}_{3}$ contained a mixture of two phases (the proportions depending on the synthetic approach used), perhaps explaining some of the apparent confusion over its exact structure.[48] The ${ }^{23} \mathrm{Na}$ MAS NMR spectra of two samples of $\mathrm{NaNbO}_{3}$, prepared using different synthetic methods, shown in Figure 12a appear very similar (with the broad spectral lineshapes resulting from the quadrupolar interaction). However, MQMAS experiments (shown in Figure 12b) reveal significant differences between the two samples - one containing two 
major signals, the other four distinct Na sites. DFT calculations using a range of proposed structural models suggested the synthetic sample contained both the well-known $\mathrm{Pbcm}$ phase and a second polymorph, with space group $P 2{ }_{1} m a$. This polar space group, resulting from a different tilting of the $\mathrm{NbO}_{6}$ octahedra (Figure 12c), provides the possibility for ferroelectric behavior, of interest in a range of industrial applications. The two polymorphs were extremely difficult to distinguish initially by diffraction, with differences primarily found in the superstructure peaks. A later study showed that ${ }^{93} \mathrm{Nb}$ NMR was not able to distinguish the two very similar polymorphs,[49] although the metastable ilmenite $\mathrm{NaNbO}_{3}$ polymorph has a very different NMR spectrum.[102] Firstprinciples calculations revealed a relatively poor correlation between the calculated ${ }^{93} \mathrm{Nb}$ $\mathrm{C}_{\mathrm{Q}}$ and shear or longitudinal strain parameters describing the distortion of the surrounding $\mathrm{NbO}_{6}$ octahedra, although a simple electrostatic point charge model was able to estimate the relative magnitude of the ${ }^{93} \mathrm{Nb} \mathrm{C}_{\mathrm{Q}}$.

Cation substitution can result not only in a structural change, but also changes in the physical and chemical properties, which can be exploited industrially and commercially. As described in Section 4, an NMR study of $\mathrm{Li}_{x} \mathrm{Na}_{1-x} \mathrm{NbO}_{3}(0.08 \leq \mathrm{x} \leq 0.20)$ revealed mixtures of a polar orthorhombic phase $\left(P 2_{1} m a\right)$ and a rhombohedral phase $(R 3 c)$, with the relative fractions dependent on synthetic conditions. ${ }^{23} \mathrm{Na}$ MQMAS NMR spectra also revealed the slow transformation of the orthorhombic phase to the rhombohedral phase on standing in air at ambient temperature.[50] At higher values of $x(0.25 \leq x \leq 0.90)$ two rhombohedral phases coexisted, one Na-rich and the other Li-rich, with ${ }^{23} \mathrm{Na}$ signals for the two clearly resolved using MQMAS, despite the spectral broadening resulting from cation disorder. $\mathrm{LiNbO}_{3}$ can be prepared using a solid-state reaction and forms a rhombohedral (non-perovskite) structure. However, recent work has shown that hydrothermal synthesis (i.e., the reaction between $\mathrm{Nb}_{2} \mathrm{O}_{5}$ and aqueous $\mathrm{LiOH}$ at $240{ }^{\circ} \mathrm{C}$ ) produces a material with a disordered rock salt structure, with the metals statistically distributed over the cation sites.[103] ${ }^{23} \mathrm{Na}$ NMR clearly showed a distribution of NMR parameters and, therefore, disorder in the material. Annealing this material produced ordered $\mathrm{Li}_{3} \mathrm{NbO}_{4}$, confirmed using XRD, ${ }^{23} \mathrm{Na}$ NMR and DFT calculations. Similar 
hydrothermal reactions were shown to produce $\mathrm{NaCe}_{1-\mathrm{x}} \mathrm{La}_{\mathrm{x}} \mathrm{Ti}_{2} \mathrm{O}_{6}(0<\mathrm{x}<1)$ perovskites, and the paramagnetic broadening in ${ }^{23} \mathrm{Na}$ MAS NMR spectra used to demonstrate that the samples were a genuine solid solution, rather than simply physical mixtures of the two oxides. The spectra revealed no evidence for A site ordering. ${ }^{2} \mathrm{H}$ NMR was also used to study samples prepared in $\mathrm{D}_{2} \mathrm{O}$ and showed evidence for A site water incorporation, accommodated by the oxidation of some $\mathrm{Ce}^{3+}$ to $\mathrm{Ce}^{4+} \cdot[104]$

Recent work by Grey and co-workers[105] used multinuclear solid-state NMR spectroscopy and first-principles DFT calculations to study dry and hydrated yttriumsubstituted $\mathrm{BaSnO}_{3}$ perovskite, a material of interest as a protonic conductor. This revealed complex cation and oxygen vacancy disorder, which varied depending on the level of yttrium substitution. The ${ }^{119} \mathrm{Sn}$ chemical shift was sensitive to the number of neighbouring $\mathrm{Sn}$ and $\mathrm{Y}$ cations (with spectral assignment confirmed using DFT calculations). Spectral intensities confirmed that there was random substitution of $\mathrm{Y}$ onto the Sn lattice up to $\mathrm{x}=0.2$, while at higher substitution levels $\mathrm{Y}-\mathrm{O}-\mathrm{Y}$ linkages were avoided, leading to complete ordering, i.e., Y-O-Sn alternation, at $\mathrm{x}=0.5$. Ordering was also confirmed by the absence of a peak in the ${ }^{17} \mathrm{O}$ spectrum that could be assigned to Y-OY environments. Resonances corresponding to five-coordinate Sn and Y were observed, suggesting that the vacancies associate, at least to some extent, with both sites, although a greater proportion of five-coordinate $S n$ was present. These peaks disappeared upon hydration of the material. High-temperature measurements indicated significant oxygen mobility above $400{ }^{\circ} \mathrm{C}$, even for materials with high $\mathrm{Y}$ content. This appeared to be a result of the cation ordering preventing proton trapping at $\mathrm{Y}-\mathrm{O}-\mathrm{Y}$ linkages.

For some metal oxides more significant disorder is observed, with considerable variation in the atomic positions. Recent work on $\gamma-\mathrm{Ga}_{2} \mathrm{O}_{3}$ used a combination of diffraction- and spectroscopy-based techniques, accompanied by reverse Monte Carlo (RMC) modelling, to investigate the long-range structure and local disorder in this material.[106] Although Rietveld refinement was able to show that Ga occupies two pairs of octahedral and tetrahedral sites (ideal spinel and non-spinel), it was not able to provide 
information on their local distribution. However, the short Ga-Ga contact produced if neighbouring spinel and non-spinel sites are simultaneously occupied ensures the local distribution is not statistical. To understand the local structure a $6 \times 6 \times 6$ supercell of $\gamma$ $\mathrm{Ga}_{2} \mathrm{O}_{3}$ was generated and refined against neutron total scattering data using an RMC approach, enabling long-range and local structure to be considered. This revealed substantial amounts of local distortion in the octahedral sites, causing the structure to closely resemble $\beta-\mathrm{Ga}_{2} \mathrm{O}_{3} .{ }^{71} \mathrm{Ga}$ MAS NMR spectra of $\gamma-\mathrm{Ga}_{2} \mathrm{O}_{3}$ (crystallite size $\sim 30 \mathrm{~nm}$ ) showed a $\mathrm{Ga}^{\mathrm{IV}} / \mathrm{Ga}^{\mathrm{VI}}$ ratio consistent with the results obtained from RMC. However, for nanocrystalline $\gamma-\mathrm{Ga}_{2} \mathrm{O}_{3}$ (crystallite size 5-15 nm), different analytical approaches gave conflicting results - Bragg diffraction and TEM suggested a greater proportion of $\mathrm{Ga}^{\mathrm{IV}}$ than $\mathrm{Ga}^{\mathrm{VI}}$, while ${ }^{71} \mathrm{Ga}$ NMR suggests all crystalline and nanocrystalline samples contain the same ratio of occupied tetrahedral and octahedral sites. The NMR spectra were in agreement with neutron scattering results that show the same average Ga-O distance regardless of particle size. This apparent inconsistency was rationalised by proposing that, in the nanocrystalline samples, an increased proportion of occupied tetrahedral sites in the bulk is offset by an increased number of occupied octahedral sites on the surface (owing to termination by bound solvent or hydroxide).

The identification and subsequent characterisation of dynamic disorder, i.e., atomic/molecular motion, is particularly important for oxides, as these phenomena often result in desirable properties, such as proton transport or electrical conductivity. This can be difficult by diffraction-based techniques, whereas if motion is present (rather than simple positional disorder) the solid-state NMR spectra often show significant changes with a small variation in temperature. The study of dynamics using NMR spectroscopy has been shown in a wide range of oxides and ceramics, and despite the added difficulties associated with studying quadrupolar nuclei, ${ }^{17} \mathrm{O}$ NMR spectroscopy has provided particularly useful insight.[4,6,94,107-109] This can be demonstrated by recent work on $\mathrm{ZrW}_{2} \mathrm{O}_{8}$, a material that exhibits negative thermal expansion over a wide temperature range (0.3-1050 K).[110-113] The structure is composed of pairs of $\mathrm{WO}_{4}$ tetrahedra, which, at low temperatures, are ordered and point in the same direction. Above $175{ }^{\circ} \mathrm{C}$, the 
tetrahedra become disordered over two possible orientations, resulting in a time-averaged disordered structure from diffraction. Variable-temperature $\left(28-229{ }^{\circ} \mathrm{C}\right){ }^{17} \mathrm{O}$ MAS NMR spectra of ${ }^{17} \mathrm{O}$-enriched $\mathrm{ZrW}_{2} \mathrm{O}_{8}$, shown in Figure 13a, suggest that all oxygen sites are involved in some sort of exchange at high temperature (i.e., above $175^{\circ} \mathrm{C}$ ), with the four resolved peaks seen at low temperatures coalescing as the temperature is increased. At lower temperatures line broadening of all four peaks also suggests some motion is present, but it is not possible to determine which sites are in exchange from the MAS spectra alone. Two-dimensional exchange or EXSY experiments (where cross peaks between species in a two-dimensional spectrum indicate the two are involved in an exchange process), shown in Figure 13a confirm exchange between all $\mathrm{O}$ sites is observed even at low temperature. Thus, ${ }^{17} \mathrm{O}$ NMR is able to distinguish between the two possible mechanisms for $\mathrm{WO}_{4}$ reorientation shown in Figure $13 \mathrm{~b}$; an $\mathrm{S}_{\mathrm{N}}$ 2-like process that interconverts $\mathrm{O} 3$ and $\mathrm{O} 4$ species only, and a "ratchet-like" mechanism where all oxygens would be affected.

Given the prominent role dynamics plays in lithium-ion battery technology, identifying and characterizing lithium dynamics in inorganic materials is of significant importance. With both ${ }^{6} \mathrm{Li}$ and ${ }^{7} \mathrm{Li} \mathrm{NMR}$ active, it is not surprising that solid-state NMR spectroscopy has been used extensively to investigate cation motion in energy materials. For example, lithium ion conductivity in $\mathrm{Li}_{3 \mathrm{x}} \mathrm{La}_{1 / 3-\mathrm{x}}(\mathrm{Nb}, \mathrm{Ti}) \mathrm{O}_{3}$ perovskites was investigated using ${ }^{6} \mathrm{Li}$ and ${ }^{7} \mathrm{Li}$ NMR spectroscopy.[114] Powder XRD measurements revealed that the $\mathrm{La}^{3+}$ distribution in the perovskite structures is affected by cation substitution (i.e., $\mathrm{Nb}^{5+}$ for $\mathrm{Ti}^{4+}$ ); an important observation when considering that the immobile $\mathrm{La}^{3+}$ ions act as obstacles for long-range $\mathrm{Li}^{+}$motion, resulting in an adverse effect on lithium ion conductivity. When compared to the titanates, the niobates had a larger unit cell volume, fewer $\mathrm{La}^{3+}$ ions and more vacant sites, which would appear to suggest increased $\mathrm{Li}^{+}$ion dynamics should be expected. However, this was not observed experimentally, suggesting that the interaction between $\mathrm{Li}^{+}$ions and their surroundings has an effect on the conductivity. ${ }^{7} \mathrm{Li}$ NMR spectra revealed two $\mathrm{Li}^{+}$environments in $\mathrm{Li}_{3 \mathrm{x}} \mathrm{La}_{1 / 3-\mathrm{x}} \mathrm{NbO}_{3}$ - one with almost no quadrupolar coupling (20\% of the signal) and a second ( $80 \%$ of the signal) where a characteristic quadrupolar lineshape was observed (suggesting lower symmetry 
at this site). Using relaxation measurements two independent $\mathrm{Li}^{+}$dynamic processes were detected, with fast motion of $\mathrm{Li}^{+}$inside the A-cages, and slower $\mathrm{Li}^{+}$hopping from $\mathrm{A}$ cage to A cage.

Although DFT calculations are carried out on static structures at $0 \mathrm{~K}$, these can provide insight into the type of dynamics observed in a system. If motion is rapid, a timeaveraged NMR spectrum is observed. Computationally, this can be reproduced by averaging NMR parameters for each relevant static $0 \mathrm{~K}$ structure (ensuring for anisotropic interactions that the orientation of the tensor and not just the principal components are considered). For motion on slower timescales, where averaging is not complete the complex spectral lineshapes can be difficult to interpret directly, but the computed interaction tensors provide a vital input for lineshape simulation. As an example, in recent work by Kim et al. variable-temperature ${ }^{17} \mathrm{O}$ NMR spectroscopy was used to investigate dynamics in the proton conductor, $\mathrm{CsH}_{2} \mathrm{PO}_{4} \cdot[115]$ DFT calculations were able to prove that $\mathrm{H} 1$ is localized in a O-H-O hydrogen bond, but there is rapid exchange $\left(\sim 10^{7} \mathrm{~s}^{-1}\right)$ of $\mathrm{H} 2$ protons between two sites within a hydrogen bond. NMR spectra could only be simulated by considering two rate constants for rotation of the phosphate ions.

\subsection{Minerals and Clays}

Minerals are defined as naturally-occurring, stable solids with a specified chemical composition, that exhibit some aspect of long-range atomic order.[116] There are a large number of materials that are structurally- or chemically-related to minerals, which can be produced via chemical modification of a natural mineral or in an entirely synthetic approach. Some of the most common materials that fall into this category are the aluminosilicate zeolites, three-dimensional framework (or tecto-) silicates. There are $\sim 45$ naturally-occurring zeolites (formally, therefore, minerals), but a vast number of materials with different framework topologies can be produced synthetically. Although briefly mentioned in this section, NMR study of zeolites will be considered in the next section in more detail. The structural study of minerals clearly has important implications in geology and geochemistry, enabling an understanding of the physical and chemical properties of 
the surface and inner layers of our planet. However, a better insight into structureproperty relationships in minerals will also be of benefit in understanding the related materials used widely in industry.

While minerals, by definition, adopt structures with long-range order in at least some of their atomic positions, their composition often varies significantly, with extensive solid solutions formed. The bulk of the Earth's crust and mantle is composed of magnesiosilicate and aluminosilicate minerals, but elements such as $\mathrm{Fe}, \mathrm{Ca}, \mathrm{Na}, \mathrm{K}, \mathrm{F}$ and $\mathrm{H}$ also play important roles.[117] Diffraction-based measurements typically provide information on the average structure, but for many decades mineralogy has looked to NMR spectroscopy to provide an atomic-level description of local structure and insight into disorder. The use of NMR in this context has probably been one of the most influential drivers of the early development of the technique, demonstrating the level of detailed information that is available. ${ }^{29} \mathrm{Si}$ NMR spectroscopy is perhaps the most commonly-used approach, providing information on the coordination number of an atom (most typically Si or Al), the degree of silicate polymerisation (usually denoted in terms of $\mathrm{Q}^{\mathrm{n}}$, where $\mathrm{n}$ is the number of bridging oxygens), and the substitution of atoms onto NNN sites. ${ }^{27} \mathrm{Al},{ }^{17} \mathrm{O},{ }^{1} \mathrm{H}$ and ${ }^{19} \mathrm{~F}$ NMR spectroscopy have also been widely applied. For more information on NMR of minerals see Refs. [118-122].

Most minerals are composed of multicomponent solid solutions, posing a considerable challenge to determine (and perhaps even simply to describe) the structure. One of the most common substitutions found is that of $\mathrm{Al}$ into the tetrahedral silicate framework; a particular problem for X-ray diffraction owing to the isoelectronic nature of $\mathrm{Si}^{4+}$ and $\mathrm{Al}^{3+}$. NMR spectroscopy has been widely applied to determine the extent and nature of $\mathrm{Si} / \mathrm{Al}$ ordering in many minerals, with the ${ }^{29} \mathrm{Si}$ isotropic chemical shift changing by $~ 6$ ppm for each NNN Al substituted.[118-122] In principle, the relative intensities of the spectral resonances can then provide information on the Si/Al ordering, as described (more generally) in Section 4. However, it has been shown in many cases that the Si/Al distribution is not random and Löwenstein's rule of aluminium avoidance holds, i.e., Al- 
O-Al linkages do not occur. In this case, the equations in Section 4 can be simplified, and it is possible to determine the $\mathrm{Si} / \mathrm{Al}$ ratio of a material directly from the ${ }^{29} \mathrm{Si}$ MAS NMR spectrum using

$$
\mathrm{Si} / \mathrm{Al}=\frac{\sum_{\mathrm{n}=0}^{4} \mathrm{I}(\operatorname{Si}(\mathrm{nAl}))}{0.25 \sum_{\mathrm{n}=0}^{4} \mathrm{n} \mathrm{I}(\operatorname{Si}(\mathrm{nAl}))},
$$

where $\mathrm{I}(\mathrm{Si}(\mathrm{nAl}))$ represents the intensity of the ${ }^{29} \mathrm{Si}$ resonance corresponding to tetrahedral Si surrounded by $\mathrm{n} \mathrm{Al.}[4,123]$ Although a number of cases have been found where there is some violation of Löwenstein's rule, this is often minimal in low-temperature phases, but can be significant for materials that crystallize from melts or glasses. One of the most famous examples of this is $\mathrm{Mg}$-cordierite $\left(\mathrm{Mg}_{2} \mathrm{Al}_{4} \mathrm{Si}_{5} \mathrm{O}_{18}\right)$, where the $\mathrm{Si} / \mathrm{Al}$ ratio determined from NMR spectroscopy differs from that determined using chemical analysis. The comparison of the two, however, does enable the number of Al-Al linkages to be determined. For zeolites, ordering beyond aluminium avoidance has been studied, with Dempsey's rule (the minimisation of Al-O-Si-O-Al linkages) suggested, as described in the next section.[120,124] A recent study of Al/Si disorder combining NMR spectroscopy and DFT calculations was undertaken by Florian et al., using ${ }^{29} \mathrm{Si}$ MAS and ${ }^{27} \mathrm{Al}$ MAS and MQMAS NMR spectroscopy.[125] This work concerned gehlenite $\left(\mathrm{Ca}_{2} \mathrm{Al}_{2} \mathrm{SiO}_{7}\right)$, which contains two $\mathrm{T}$ sites, one occupied by $\mathrm{Al}$ and the second by Si or Al. This results in two different possible environments for Si and seven for $\mathrm{Al}$ (resolved using MQMAS and assigned using multinuclear two-dimensional experiments and by comparison to DFT calculations for a series of 50 models based on supercells of gehlinite with $\mathrm{Si}$ and Al substituted onto different sites). The relative intensities of the resonances in both ${ }^{29} \mathrm{Si}$ and ${ }^{27} \mathrm{Al}$ spectra reveal a deviation from Löwenstein's rule, and a comparison of the width of the spectral lines with the calculated parameters enabled the distribution of geometrical parameters (i.e., T-O-T bond angles) to be determined.

Although $\mathrm{Si} / \mathrm{Al}$ substitution is perhaps the most common form of disorder studied in minerals, a number of other cation and anion substitutions are also of importance, and have been studied using NMR spectroscopy. One example is $\mathrm{Mg} / \mathrm{Ca}$ ordering in garnets, a 
group of minerals with a general formula $\mathrm{X}_{3} \mathrm{Y}_{2} \mathrm{Si}_{3} \mathrm{O}_{12}$, which are common in metamorphic rocks and in the mantle. Early ${ }^{29} \mathrm{Si}$ NMR of solid solutions between pyrope $(\mathrm{X}=\mathrm{Mg}, \mathrm{Y}=$ $\mathrm{Al})$ and grossular $(\mathrm{X}=\mathrm{Ca}, \mathrm{Y}=\mathrm{Al})$ showed evidence of non-random $\mathrm{Mg} / \mathrm{Ca}$ distributions, with small changes in the peak intensities observed for some samples when synthesis was carried out at a different temperature.[126] More recently, ${ }^{17} \mathrm{O}$ MQMAS was able to resolve four distinct $\mathrm{O}$ environments, two corresponding to $\mathrm{O}$ bonded to $2 \mathrm{Mg}$ and $2 \mathrm{Ca}$ (i.e., as seen in end-member garnets) and two where $\mathrm{O}$ is bonded to $1 \mathrm{Ca}$ and $1 \mathrm{Mg}$. The change in peak position as the $\mathrm{Mg}$ content increases was related to a shortening of the $\mathrm{Ca}-\mathrm{O}$ bond length. In 2015, Palke et al. extended work in this area,[127] using ${ }^{29} \mathrm{Si}$ and ${ }^{27} \mathrm{Al} \mathrm{NMR}$ to study cation disorder in Fe-containing garnets. The interaction with unpaired electrons on the substituted Fe results in large paramagnetic shifts of the spectral resonances, enabling information on the Fe distribution to be obtained.

In different types of minerals there is the possibility of more significant cation and anion disorder. For example, phyllosilicates (or sheet silicates) are formed from parallel sheets of $\mathrm{SiO}_{4}$ tetrahedra, joined by sheets of octahedrally-coordinated cations.[116] Phlogopite $\left(\mathrm{KMg}_{3}\left(\mathrm{AlSi}_{3}\right) \mathrm{O}_{10}(\mathrm{OH}, \mathrm{F})_{2}\right)$ from the mica family of layered silicates, has been extensively studied by NMR spectroscopy, investigating Si/Al disorder within sheets, substitution of $\mathrm{Mg} / \mathrm{Si}$ for $\mathrm{Al}$ (according to Tschermak's substitution (where $\mathrm{Mg}^{\mathrm{VI}}$ and $\mathrm{Si}^{\mathrm{iV}}$ are replaced by $\mathrm{Al}^{\mathrm{VI}}$ and $\left.\mathrm{Al}^{\mathrm{IV}}\right)$ ) and $\mathrm{OH}^{-} / \mathrm{F}^{-}$disorder on the anion lattice. In recent work, Langner et al. combined NMR spectroscopy and Monte Carlo simulations to study cation ordering in Al-rich phlogopites.[128] The work established that the cation ordering in the tetrahedral sheets obeyed Löwenstein's rule (i.e., aluminium avoidance) but showed segregation of $\mathrm{Al}$ in the octahedral layers, with a preference for $\mathrm{Al}^{\mathrm{VI}}$ and $\mathrm{Al}^{\mathrm{IV}}$ to occupy neighboring sites. This results in a separation into clusters of phlogopite $\left(\mathrm{KMg}_{3}\left(\mathrm{AlSi}_{3}\right) \mathrm{O}_{10}(\mathrm{OH})_{2}\right)$ and eastonite $\left(\mathrm{KMg}_{2} \mathrm{Al}\left(\mathrm{AlSi}_{2}\right) \mathrm{O}_{10}(\mathrm{OH})_{2}\right)$ regions, as shown in Figure $14 \mathrm{a}$, the configuration of neighboring tetrahedral and octahedral sheets produced from Monte Carlo simulations. The computational results were in good agreement with experiments, with the ${ }^{1} \mathrm{H} /{ }^{29} \mathrm{Si}$ HETCOR spectrum shown in Figure 14a, where the spectral intensities confirm that Al-rich Si environments in the tetrahedral sheet are more likely to 
be found in close spatial proximity to Al-rich H environments in the octahedral sheets. The work of Fechtelkord and Langner in 2013 investigated the effects of $\mathrm{OH}^{-} / \mathrm{F}^{-}$substitution in the octahedral layer, using ${ }^{1} \mathrm{H} /{ }^{29} \mathrm{Si}$ and ${ }^{19} \mathrm{~F} /{ }^{29} \mathrm{Si} \mathrm{CP}$ experiments.[129] These showed that $\mathrm{F}^{-}$ is usually located in Mg-rich octahedral and Si-rich tetrahedral clusters, while $\mathrm{OH}^{-}$are found in Al-rich octahedral and tetrahedral regions. Thus, the incorporation of $\mathrm{F}^{-}$into phlogopite significantly reduced the capacity of the structure to incorporate Al.

Clay minerals are a very important group of phyllosilicates, all of which contain tetrahedrally- and octahedrally-coordinated cations, and which can be differentiated into different groups by their layer spacings.[116] NMR has played a significant role in the study of clays (and related layered materials), providing information on the ordering of cations within the layers, the presence and distribution of any charge-balancing anions, and the nature and position of the interlayer material.[120-122] One of the most studied clay minerals is montmorillonite, which has general formula $(\mathrm{Na}, \mathrm{Ca})_{0.33}(\mathrm{Al}, \mathrm{Mg})_{2}\left(\mathrm{Si}_{4} \mathrm{O}_{10}\right)(\mathrm{OH})_{2} \cdot \mathrm{nH}_{2} \mathrm{O}$. A detailed $\mathrm{NMR}$ investigation into synthetic and natural montmorillonite was undertaken by Cadars et al.[130] ${ }^{29}$ Si MAS NMR spectra were dominated by a broad, intense peak at $-93.7 \mathrm{ppm}\left(\mathrm{Q}^{3}\right)$, but contained a smaller shoulder at -88.6 ppm (assigned to $\mathrm{Q}^{3}(1 \mathrm{Al})$ ), suggesting substitution of $\mathrm{Al}$ into the tetrahedral layers. This was confirmed by the presence of $\mathrm{Al}^{\mathrm{IV}}$ and $\mathrm{Al}^{\mathrm{VI}}$ species in the ${ }^{27} \mathrm{Al}$ MAS NMR spectrum, and by ${ }^{27} \mathrm{Al}\left\{{ }^{29} \mathrm{Si}\right\}$ dipolar correlation (HMQC) spectroscopy, where a strong correlation of $\mathrm{Al}^{\mathrm{VI}}$ and $\mathrm{Si} \mathrm{Q}^{3}$ species was observed (i.e., between layers), along with a second correlation between the $\mathrm{Q}^{3}(1 \mathrm{Al}){ }^{29} \mathrm{Si}$ and $\mathrm{Al}^{\mathrm{IV}} \cdot{ }^{1} \mathrm{H} \mathrm{NMR}$ was able to distinguish between $\mathrm{Al}_{2} \mathrm{OH}, \mathrm{MgAlOH}$, inter-layer water and adsorbed water. The ${ }^{29} \mathrm{Si} \mathrm{NMR}$ spectrum showed the presence of a broad component at $-106 \mathrm{ppm}$, corresponding to a silicate phase with little or no order (and not observed by XRD). Further analysis of all NMR spectra enabled the $\mathrm{Si} / \mathrm{Al}$ and $\mathrm{Mg} / \mathrm{Al}$ ratios to be determined, and the composition of the clay to be revised. The assignment of all spectra was confirmed using DFT calculations of a number of structural models with differing cation arrangements. Calculated ${ }^{1} \mathrm{H}$ chemical shifts contradicted the commonly-accepted relationship that the variation of the ${ }^{1} \mathrm{H}$ chemical shift in clay minerals depends primarily on the orientation of the OH group (and, 
hence, the hydrogen bond strength), demonstrating that $\mathrm{Mg} / \mathrm{Al}$ substitution at neighbouring sites resulted in a significant ${ }^{1} \mathrm{H}$ chemical shift. The DFT energies suggested a non-random distribution of $\mathrm{Mg} / \mathrm{Al}$ in the octahedral layer, with adjacent $\mathrm{Mg}$ atoms present in only small amounts. A non-random cation distribution was also observed using NMR spectroscopy in layered double hydroxides (LDHs), a family of anionic clays.[131] Fast ${ }^{1} \mathrm{H}$ MAS (i.e., $\sim 60 \mathrm{kHz}$ ) was used to distinguish and quantify different local $\mathrm{Mg}_{\mathrm{n}} \mathrm{Al}_{3-}$ ${ }_{n} \mathrm{OH}(\mathrm{n}=1,2$ and 3$)$ environments. The ${ }^{1} \mathrm{H}$ and ${ }^{27} \mathrm{Al}$ NMR spectra were assigned using DFT calculations. The relative proportions of each species (from integration of the assigned spectral resonances) suggested the clay was not perfectly ordered, with small amounts of Al clustering detected, demonstrating that, although not favoured, Al-O-Al linkages are not completely excluded in Al-rich LDHs.

NMR spectroscopy can also provide information on the chemical behavior of minerals, e.g., their response to thermal treatment or reaction, and the intercalation or grafting of small molecules (particularly for layered materials). In the latter case, NMR is used to determine the nature and proportion of small molecules present and to determine whether a molecule is grafted (i.e., a covalent bond is formed) or merely intercalated. An elegant example of this was provided by Senker and co-workers,[132] in 2011, who used multinuclear NMR spectroscopy to study the grafting of ethylene glycol to kaolinite (a clay mineral with formula $\mathrm{Al}_{2} \mathrm{Si}_{2} \mathrm{O}_{5}(\mathrm{OH})_{4}$, used to produce paper, ceramics, cosmetics and paint). Grafting, rather than intercalation, of ethylene glycol onto the $\mu$-bridged $\mathrm{OH}$ was confirmed using ${ }^{27} \mathrm{Al}$ MQMAS NMR (which showed a considerable change in the local Al environment) and ${ }^{13} \mathrm{C} /{ }^{27} \mathrm{Al}$ REAPDOR measurements (which showed a $\mathrm{C}-\mathrm{Al}{ }^{\mathrm{VI}}$ distance of $3.1 \AA$ ). The dynamic disorder of the grafted molecule (i.e., its orientation between the clay layers) was also studied using ${ }^{1} \mathrm{H}$ NMR. The thermal behavior of kaolinite has also been the subject of much study by NMR, with heat treatment shown to initially lead to dehydroxylation before producing metakaolinite. This amorphous material is difficult to study by diffraction, but does retain some long-range order in the stacking of the clay layers, and is characterised by a ${ }^{29} \mathrm{Si}$ resonance at $-101.9 \mathrm{ppm}$ and the presence of four-, five- and six-coordinate Al species. Figure $14 \mathrm{~b}$ shows ${ }^{27} \mathrm{Al}$ MAS NMR spectra of kaolinite 
and the same sample calcined at varying temperatures.[133] The decomposition of kaolinite to metakaolinite begins at $\sim 500{ }^{\circ} \mathrm{C}$, with loss of all kaolinite at $650{ }^{\circ} \mathrm{C}$. The spectrum of metakaolinite contains three main peaks at 4, 25, and 53 ppm (assigned to six-, five-, and four-coordinated Al), with a broad, low-frequency tail extending down to ca. 200 ppm. Above $950{ }^{\circ} \mathrm{C}$, metakaolinite transforms to amorphous material, mullite and some $\gamma$ $\mathrm{Al}_{2} \mathrm{O}_{3}$, with the majority of $\mathrm{Al}$ having four- and six-coordinate environments.

As described in Section 6.1, there is a growing interest in the use of ceramic materials for the encapsulation of Ln- and An-bearing radioactive waste. One important challenge is to gain an understanding of the effects of radiation damage on the structure and stability of such materials. Minerals such as zircon $\left(\mathrm{ZrSiO}_{4}\right)$ contain significant amounts of natural $U$ and $T h$, and so provide considerable insight into the long-term (i.e., hundreds or even millions of years) structural changes that take place.[134-136] ${ }^{29} \mathrm{Si}$ MAS NMR spectra of radiation-damaged zircons show a sharp signal at $~ 82$ ppm, resulting from a crystalline region, with a broader signal to lower ppm, attributed to amorphised regions of the sample, as shown in Figure 15.[136] Amorphisation occurs owing to the $\alpha$ decay of $U$ and $T h$, with the recoil of the heavy nucleus into the surrounding atoms upon emission of an $\alpha$ particle producing structural damage and a partially metamict structure, as shown in Figure 15.[137] The relative proportions of crystalline and amorphous regions depend upon the radiation dose (i.e., the amount of radioactive species present and the time during which decay has occurred). From quantitative ${ }^{29} \mathrm{Si}$ NMR it is, therefore, possible to determine the number of atoms permanently displaced by each $\alpha$-decay, with recent work suggesting as many as 5000-6000 atoms are displaced by a $30 \mathrm{keV}^{234} \mathrm{U}$ recoil in zircon.[134] This is a significant increase on the 1000-2000 atoms suggested by Monte Carlo simulations, but is in good agreement with MD simulations, as shown in Figure 15.[137] The nature of the amorphised region produced by radioactive decay has also attracted debate. The ${ }^{29} \mathrm{Si}$ chemical shift of the broad resonance might suggest $\mathrm{Q}^{2 / 3}$ species are present. Given the stoichiometry of $\mathrm{ZrSiO}_{4}$, the production of Si-O-Si linkages would then result in $\mathrm{O}$ atoms that are not bonded to Si. An average polymerisation of $\mathrm{Q}^{2 / 3}$ in the amorphous phase would be difficult to reconcile with charge balance and the new oxygen 
species would need to be assigned to interstitial defect sites. DFT calculations of a highpressure polymorph of $\mathrm{ZrSiO}_{4}$ (reidite), and of amorphous $\mathrm{ZrSiO}_{4}$ showed that $\mathrm{Q}^{0} \mathrm{Si}$ species could exhibit ${ }^{29} \mathrm{Si}$ shifts between -90 and $-96 \mathrm{ppm}$, thus making it difficult to unequivocally identify the extent of polymerization in the amorphous regions.[138,139] Insight was provided by ${ }^{17} \mathrm{O}$ NMR, where bridging and non-bridging oxygens could clearly be resolved, as shown in Figure 15c.[140] However, these experiments posed a considerable experimental challenge as they had to be carried out at natural abundance levels $\left(0.037 \%{ }^{17} \mathrm{O}\right)$, requiring both $\mathrm{CPMG}$ echo trains in acquisition and a pre-acquisition signal-enhancement scheme to be used to see signal on a reasonable timescale (e.g., 2.6 days for the spectrum in Figure 15c).

A change in chemical composition, from aluminosilicates to Fe-bearing magnesium silicates, signifies the boundary of the Earth's crust and mantle.[117] The mantle itself can be further subdivided into layers, according to the silicate phases present. The major component of the upper mantle is olivine $\mathrm{Mg}_{1.8} \mathrm{Fe}_{0.2} \mathrm{SiO}_{4}$, an iron-bearing form of forsterite $\left(\alpha-\mathrm{Mg}_{2} \mathrm{SiO}_{4}\right)$, while increasing pressure results in a transformation to Fe-bearing wadsleyite, $\beta-(\mathrm{Mg}, \mathrm{Fe})_{2} \mathrm{SiO}_{4}$, and ringwoodite $\gamma-(\mathrm{Mg}, \mathrm{Fe})_{2} \mathrm{SiO}_{4}$, in the upper and lower transition zone, respectively. Below this, at depths greater than $660 \mathrm{~km}$, ringwoodite disproportionates to perovskite, $(\mathrm{Mg}, \mathrm{Fe}) \mathrm{SiO}_{3}$, and magnesiowustite, $(\mathrm{Mg}, \mathrm{Fe}) \mathrm{O}$, in the lower mantle. Owing to the difficulties associated with obtaining natural mantle minerals, most NMR studies have typically focused on (Fe-free) synthetic samples produced at high pressure, resulting in very small sample volumes. A synthetic approach does, however, enable isotopic enrichment (e.g., of ${ }^{29} \mathrm{Si},{ }^{25} \mathrm{Mg}$ or ${ }^{17} \mathrm{O}$ ), significantly improving sensitivity. For a more detailed review on NMR of mantle silicates see Refs. [121,122]. In addition to characterizing the ordered Fe-free end members of each silicate phase, NMR has also been used to probe disorder in high-pressure minerals. Although Al is much less important in the mantle than in the crust, Al-rich phases may be present in subducted slabs, and low levels of $\mathrm{Al}$ substitution into mantle minerals could have important implications for the physical and chemical properties of the mantle. Palke et al.[141] used ${ }^{27} \mathrm{Al}$ NMR to show that $\mathrm{Al}$ substituted onto the tetrahedral and octahedral sites (i.e., for $\mathrm{Si}$ and $\mathrm{Mg}$, 
respectively) in Al- and Fe-bearing perovskites. Substitution of Fe broadened the spectral lineshapes, and ${ }^{29} \mathrm{Si}$ signal decreased significantly for $1-2.5 \%$ substitution, but showed little further change upon higher levels of substitution. In contrast, there was little effect on the ${ }^{27} \mathrm{Al}$ MAS spectrum for substitution levels of $1-2.5 \%$, but signal was almost completely removed at $5 \%$ substitution. This suggested that $\mathrm{Al}^{3+}$ and $\mathrm{Fe}^{3+}$ occupy adjacent sites within the structure, i.e., there is a preference for coupled substitution and a non-random cation distribution.

Over the last few decades it has been realized that the nominally anhydrous minerals (NAMs) that make up the inner Earth can contain significant quantities of hydrogen, usually quoted in terms of the level of its oxide (i.e., water) present. Hydrogen is thought to be incorporated into NAMs as $\mathrm{OH}^{-}$groups, usually associated with defects in the lattice. The distribution of water in the mantle could significantly affect its properties. The difficulties of identifying $\mathrm{H}$ positions, and in particular when these are disordered, using diffraction, has led to significant interest in the use of NMR spectroscopy to provide information on the structure, disorder and dynamics present in NAMs. Early work typically used ${ }^{1} \mathrm{H}$ NMR,[142,143] as the inherently high sensitivity of this nuclide is ideal for probing low $\mathrm{OH}$ concentrations. Also of interest was the observation that the ${ }^{1} \mathrm{H}$ chemical shift showed a strong correlation with the strength of hydrogen bonding interaction, allowing the $\mathrm{O}-\mathrm{H}$...O bond lengths to be determined directly from the spectrum, for each species independently.[144] Work by Kohn and Keppler[142-144] estimated the solubility of $\mathrm{H}_{2} \mathrm{O}$ in a range of minor and major mantle silicates, with the water content determined from ${ }^{1} \mathrm{H}$ NMR peak intensities. The latter work suggested that for samples prepared in the same way, broadly similar $\mathrm{H}_{2} \mathrm{O}$ concentrations were obtained by ${ }^{1} \mathrm{H}$ NMR and FTIR ( 150 ppm for forsterite and 200 ppm for enstatite), although $\mathrm{H}_{2} \mathrm{O}$ concentrations were found to vary by orders of magnitude if samples were prepared and / or treated differently.

The difficulties associated with studying low levels of $\mathrm{H}$ in NAMs has resulted in interest in minerals that contain stoichiometric proportions of $\mathrm{H}$ as models for the hydrous 
defects present in mantle minerals. One family of minerals that has attracted attention is the humites, which have been widely studied using NMR spectroscopy. These minerals have general formula $\mathrm{nMg}_{2} \mathrm{SiO}_{4} \cdot \mathrm{Mg}(\mathrm{OH})_{2}$, where $\mathrm{n}=1-4$, and can be viewed as hydrated forms of forsterite, consisting of $\mathrm{n}$ forsterite-like layers separated by a layer of brucite $\left(\mathrm{Mg}(\mathrm{OH})_{2}\right) \cdot[116]$ Diffraction confirms these phases are disordered, with two distinct $\mathrm{H}$ sites associated with each hydroxyl group, denoted H1 and H2, as shown in Figure 16a, each of which has an occupancy of 50\%. However, adjacent H1 sites are separated only by $\sim 1 \AA$ and are not occupied simultaneously. ${ }^{17} \mathrm{O}$ MQMAS experiments (of minerals enriched to $35 \%$ in $\left.{ }^{17} \mathrm{O}\right)$ resolved 5 resonances for clinohumite $(\mathrm{n}=4)$, as shown in Figure $16 \mathrm{~b}$, although their relative intensities suggested that the 8 expected silicate $O$ species were observed. (Note the hydroxyl O species was not detected using MQMAS in this early work, but was identified using static CP experiments).[145] To assign the spectrum DFT calculations were carried out on structural models with different arrangements of the $\mathrm{OH}$ groups within the unit cell.[146] The spectrum produced by summing the results together (i.e., assuming the disordered material contained all these local environments) was in poor agreement with experiment, while a spectrum simulated assuming fast exchange of $\mathrm{OH}$ groups (i.e., averaging of the NMR parameters for each $\mathrm{O}$ species between the different models) produced a much better result, as shown in Figure 16b. This suggests a dynamic, not a static, distribution of $\mathrm{OH}$ groups in clinohumite, a result verified using STMAS, where comparison of the experimental spectrum with those simulated using the DFT calculated NMR parameters (Figure 16c), and assuming exchange of H positions, was able to determine a rate constant, $\mathrm{k}$, (at room temperature) of $3.2 \times 10^{5} \mathrm{~s}^{-1}$.[146] The presence of dynamic disorder in clinohumite was also confirmed using ${ }^{2} \mathrm{H}$ NMR of a deuterated sample, with the linebroadening observed in the MAS spectrum (and shown in Figure 16d), confirming the presence of microsecond-timescale dynamics, and enabling an activation energy of $40 \mathrm{~kJ} \mathrm{~mol}^{-1}$ to be estimated from an Arrhenius plot.[147]

The substitution of hydroxyl groups for fluoride ions occurs commonly in nature for humites. For $\mathrm{OH}^{-}$groups adjacent to $\mathrm{F}^{-}$ions, occupancy of the $\mathrm{H} 1$ site is favoured due to the formation of a O-H...F hydrogen bond, as shown in Figure 16a. Diffraction data for 
50\%-substituted minerals have been interpreted as having full occupancy of a single $\mathrm{H} 1$ site and, thus, as fully ordered. However, ${ }^{19} \mathrm{~F}$ NMR of such a sample showed four distinct resonances (as shown in Figure 16e), perhaps suggesting some disorder.[86] Assignment of the spectrum was carried out using J-resolved spectroscopy (where J coupling between species can be measured in a two-dimensional spectrum), and DFT calculations of both chemical shifts and J couplings, in a series of models with different positions and levels of F substitution in a supercell of clinohumite. As shown in Figure 16e, F resonances at high chemical shift result from species in $\mathrm{F}$...H-O hydrogen bonds, but the species labeled $\mathrm{F}_{\mathrm{A}}$ and $\mathrm{F}_{\mathrm{B}}$ differ in the ordering of the next hydrogen bond along the chain (i.e., $\mathrm{F}$...H-O or O$\mathrm{H}$...F). In the case of $\mathrm{F}-\mathrm{Mg}-\mathrm{F} \ldots \mathrm{HO}$ linkages $\left(\mathrm{F}_{\mathrm{B}}\right)$, a surprising $\mathrm{J}$ coupling between the two F species was observed, demonstrated using DFT to have a considerable "through space" component, with the two F species separated by a distance (2.7 $\AA$ ) slightly greater than the sum of the ionic radii.[86] However, the two species at lower shift, $F_{C}$ and $F_{D}$, can only result from F-rich regions of the mineral, suggesting much more significant $\mathrm{F}^{-} / \mathrm{OH}^{-}$ disorder than observed by diffraction.

Significantly higher levels of water can be stored in the transition-zone minerals, with wadsleyite and ringwoodite able to incorporate up to $\sim 3.3 \%$ by weight, with $\mathrm{H}$ substitution thought to be charge-balanced by a Mg vacancy.[117] A number of theoretical studies have suggested protonation of the $\mathrm{O} 1$ site - an unusual $\mathrm{O}$ species coordinated by five Mg,[121] and Smyth suggested an ordered model for fully hydrous wadsleyite, with O1 protonation charge balanced by Mg2 vacancies.[148] However, a range of other studies suggested favourable protonation sites for all four $\mathrm{O}$ species. Early ${ }^{1} \mathrm{H}$ NMR of hydrous wadsleyite (up to 1.5\%) suggested more much disorder, with between 4 and $14 \mathrm{H}$ sites suggested, depending on the hydration level.[149] A detailed study of hydrous wadsleyite, combining NMR and DFT calculations, was carried out by Griffin et al. ${ }^{17} \mathrm{O}$ high-field (20.0 T) MAS and STMAS spectra of a sample with a water content of $3 \%$ showed a significant decrease in the intensity of the $\mathrm{O} 1$ resonance, and an increase in a resonance consistent with the formation of $\mathrm{Mg}-\mathrm{OH}$ groups, confirming the majority of protonation was at O1.[150] However, broadening of the O3 and O4 (non-bridging) 
lineshapes, also confirmed a disordered distribution of $\mathrm{H}$ sites. ${ }^{1} \mathrm{H}$ NMR showed a resonance at $\sim 4 \mathrm{ppm}$, consistent with $\mathrm{Mg}-\mathrm{OH}$ protons (i.e., protonation of $\mathrm{O} 1$ ), and lower intensity resonances between 6 and $10 \mathrm{ppm}$. These were attributed to Si-OH groups using ${ }^{1} \mathrm{H} /{ }^{29} \mathrm{Si}$ two-dimensional correlation experiments, while ${ }^{1} \mathrm{H} /{ }^{17} \mathrm{O}$ correlation experiments confirmed two different types of $\mathrm{OH}$ species were present. DFT calculations for fully hydrated model structures revealed that best agreement with the experimental ${ }^{17} \mathrm{O} N M R$ lineshape was observed when hydration was balanced by $\mathrm{Mg} 3$ vacancies, and poor agreement with experiment was observed for the structure proposed by Smyth (with $\mathrm{Mg} 2$ vacancies). The assignment of resonances to $\mathrm{Mg}-\mathrm{OH}$ and $\mathrm{Si}-\mathrm{OH}$ species was also supported by DFT calculations, leading to an estimation of $85 \%$ of protonation occurring at $\mathrm{O} 1$, and $15 \%$ at silicate oxygens. As described in Section 5, and shown in Figure 10, AIRSS studies[93] have suggested that protonation of O1 is most favourable, with an increase in enthalpy required for protonation of $\mathrm{O} 3$ and $\mathrm{O} 4$ sites, and the NMR parameters calculated for these structures are in excellent agreement with the experimental results.

\subsection{Microporous Materials}

Microporous materials, containing molecule-sized (5-20 A) channels and pores, are used in areas as diverse as gas storage or purification, drug delivery, catalysis, construction materials, data storage, chemical sensing, medicine and as a protecting-group strategy in organic synthesis.[151] Microporosity can be exhibited by a wide range of materials, ranging from the wholly inorganic (aluminosilicate zeolites, aluminophosphates (AlPOs), silicoaluminophosphates (SAPOs), metal-doped AlPOs (MAPOs), gallohpsphates (GaPOs), etc.), to organic-inorganic (e.g., metal-organic frameworks (MOFs) and coordination polymers) to wholly organic (covalent organic frameworks (COFs), etc.). Much effort has been devoted to characterising the structures of these types of materials and relating this to their behaviour. For further detail on the application of solid-state NMR spectroscopy to microporous materials, readers are directed to the more general reviews in Refs. [2,108, 152-162]. 
Solid-state NMR spectroscopy is routinely used to characterise inorganic microporous frameworks, often simply providing support for a crystal structure determined from Bragg diffraction. However, in many of these materials, compositional, positional, or temporal disorder exists and, as discussed above, solid-state NMR spectroscopy is uniquely placed to study these. The most well-known microporous frameworks are the aluminosilicate zeolites, some of which occur naturally as minerals, as described above, and many of which are now used industrially as highly selective catalysts. Aluminosilicates are based on the parent $\mathrm{SiO}_{2}$ composition, with the substitution of $\mathrm{Al}^{3+}$ for $\mathrm{Si}^{4+}$ leading to an overall negative charge on the framework. This can be balanced by the inclusion of cations within the pores (either organic structure-directing agents (SDAs) or metal cations), or Brønsted-acidic sites on the framework itself (i.e., protonation of one of the framework oxygen sites). As discussed above, when Löwenstein's rule of Al-O-Al avoidance holds, the amount of $\mathrm{Al}$ present in the framework can be quantified from quantitative ${ }^{29} \mathrm{Si}$ NMR spectroscopy.[4] However, particularly in zeolites with a Si/Al ratio of close to unity, Löwenstein's rule can be violated. For example, in Linde type A (LTA) zeolite (with $\mathrm{Al} / \mathrm{Si}=1$ ), rather than containing exclusively $\mathrm{Si}(\mathrm{OAl})_{4}$ species (i.e., Löwenstein-type ordering), $\mathrm{Si}(\mathrm{OAl})_{3}(\mathrm{OSi})$ species were observed by ${ }^{29} \mathrm{Si}$ NMR spectroscopy, thereby also implying the existence of $\mathrm{Al}(\mathrm{OAl})(\mathrm{OSi})_{3}$ species.[163,164] The "forbidden" Al-O-Al linkages can also be observed directly by ${ }^{17} \mathrm{O}$ NMR spectroscopy, as demonstrated by Stebbins et al.[165] for natural stilbite, enriched by exchange with ${ }^{17} \mathrm{OH}_{2}$. The Al-O-Al species has a higher chemical shift than either Al-O-Si or Si-O-Si, making it readily distinguishable in an MQMAS NMR spectrum.

The universal validity of Dempsey's rule of Al-O-Si-O-Al avoidance discussed briefly above is unclear for zeolites as, in many framework types, these linkages appear allowed, or even favoured.[120,124] In one particularly detailed study, Melchior et al.[166] used post processing to improve the resolution of the ${ }^{29} \mathrm{Si}$ MAS NMR spectra of FAU-type aluminosilicates with $\mathrm{Si} / \mathrm{Al}$ ratios of 1.28 to 5.31. The authors showed that Al-O-Si-O-Al linkages were strongly disfavoured when the two Al atoms were in the same double sixmembered ring (D6R) unit, but this was less true when the two $\mathrm{Al}$ atoms were in different 
D6Rs. This indicates that the first stage of the synthesis is formation of the D6Rs in solution, where Dempsey's rule is followed but, when the zeolitic framework begins to form by condensation of the D6Rs, only Löwenstein's rule appears to be obeyed.

The number, distribution and strength of acid sites present in zeolites are factors crucial to their catalytic performance. Brønsted acid sites can be investigated either directly by ${ }^{1} \mathrm{H}$ NMR spectroscopy, or by the use of "probe molecules", which interact with the acid sites and provide a characteristic spectroscopic signature. For Lewis-acidic sites, the use of probe molecules is required - see the more specialist reviews in Refs. [154156,158,159] for more detail. The dynamic behaviour of molecules within zeolites can also be probed by solid-state NMR spectroscopy. As discussed above, variable-temperature ${ }^{2} \mathrm{H}$ NMR experiments are an excellent probe of dynamics on the microsecond timescale, typical of confined molecules.[64,157] In one recent example, Kolokolov et al.[167] used ${ }^{2} \mathrm{H}$ NMR spectroscopy to confirm that $\mathrm{C}_{2} \mathrm{D}_{4}$ adsorbed in $\mathrm{Ag} / \mathrm{H}-\mathrm{ZSM}-5$ was bound to the $\mathrm{Ag}^{+}$ cation in an $\eta_{2}$ mode and displayed two $C_{2}$ rotations: about the $C=C$ bond with an activation barrier of $2.3 \mathrm{~kJ} \mathrm{~mol}^{-1}$ and about the $\pi-\mathrm{Ag}$ bond with an activation energy of 4.7 $\mathrm{kJ} \mathrm{mol}^{-1}$, as shown in Figure 17. NMR spectroscopy has also been applied to more detailed studies of the reactions involving zeolites themselves, including in situ crystallisation studies[168] and post-synthetic disassembly and rearrangement, e.g., in the so-called assembly-disassembly-organisation-reassembly (ADOR) route.[169,170]

Compositionally similar to zeolites are mesoporous silicas, aluminosilicas and aluminas. These materials have larger pores, such that the guest molecules are not in such close confinement as in microporous materials, and the interactions considered are closer to molecule-surface than molecule-pore wall. NMR spectroscopy is commonly used to confirm the degree of condensation (e.g., ${ }^{29} \mathrm{Si}$ NMR spectroscopy can be used to quantify the number of $\mathrm{Q}^{3}$ and $\mathrm{Q}^{4}$ species present), the number and type of $\mathrm{OH}$ species present and whether surface modification (e.g., grafting of organosilanes) has occurred.[153,157] Recent applications of solid-state NMR spectroscopy to mesoporous silicas have often been assisted by the signal enhancement of DNP, $[44,171]$ which has allowed, for example, 
the rapid acquisition of natural-abundance two-dimensional ${ }^{1} \mathrm{H}_{-}-{ }^{13} \mathrm{C},{ }^{1} \mathrm{H}-{ }^{15} \mathrm{~N}$ and ${ }^{1} \mathrm{H}-{ }^{29} \mathrm{Si}$ correlation spectra for surface species in a functionalised mesoporous silica, at a concentration of only $0.24 \mathrm{mmol} \mathrm{mL} \mathrm{m}^{-1}$.[172] With the authors estimating a reduction in experimental times of a factor of $\sim 12700$, it is clear that DNP-enhanced experiments will open the avenue to probe even very dilute surface species in mesoporous materials by NMR spectroscopy. Beyond mesoporous materials, the study of surfaces of similar composition (e.g., in catalyst supports) has long been an aim of NMR spectroscopy, although studies have typically been hampered by the low concentration of the active sites and the fact that the signal from the bulk of the material typically dominates the spectrum. Recently, the surface-selective nature of DNP-enhanced NMR spectroscopy has been employed to great effect to both improve the absolute signal per unit time from the surface, and also its intensity relative to the bulk. This so-called surface-enhanced NMR spectroscopy (SENS) has great promise for the study of surfaces, potentially enabling the acquisition of advanced multidimensional or multinuclear experiments in a feasible timescale.[171]

Related to zeolites, and capable of forming many of the same framework types, AlPOs have a formula of $\mathrm{AlPO}_{4}$ (isoelectronic with $\mathrm{SiO}_{2}$ ) and display strict alternation of $\mathrm{AlO}_{4}$ and $\mathrm{PO}_{4}$ tetrahedra. This gives a charge-neutral framework with relatively simple ${ }^{27} \mathrm{Al}$ and ${ }^{31} \mathrm{P}$ spectra, where the number of resonances is (in principle) equal to the number of crystallographically-distinct Al or P sites. Owing to the neutral framework charge and the presence of cationic SDAs in the pores, as-made AlPOs contain framework-bound anions, leading to five- and six-coordinate $\mathrm{Al}$ species as well as the expected tetrahedral species, and many AlPOs also contain crystallographic water molecules in the pores. For ordered AlPOs, it has been demonstrated that DFT calculations have been shown to provide excellent agreement with both NMR and crystallographic experiments, provided careful optimisation of the geometry is carried out.[77,78] The success of DFT calculations in predicting the NMR parameters of AlPOs has led to the technique being adopted extensively to support many NMR-crystallographic investigations of disorder in recent years.[9,10,152,153] For example, in the SAT-type AlPO STA-2, X-ray crystallography 
determined the structure shown in Figure 18(a).[173,174] There are two cancrinite cages per unit cell, each with three possible sites for the $\mathrm{OH}^{-}$anions. These sites are equivalent by the symmetry imposed by the $R \overline{3}$ space group, but have an occupancy of 0.33 as, for charge-balancing, only two of these sites can be occupied in any one unit cell. Seymour et al. combined one- and two-dimensional NMR experiments and DFT calculations to investigate the distribution of the anions.[174] The NMR parameters were calculated for a series of structural models with different $\mathrm{OH}$ sites occupied each time. The calculated ${ }^{31} \mathrm{P}$ and ${ }^{27} \mathrm{Al}$ isotropic shifts were compared with experimental J-HETCOR spectra (using the nominally through-bond INEPT magnetisation transfer), as shown in Figure 18(b), and it was possible to show that models with two $\mathrm{OH}^{-}$located in the same cancrinite cage were not observed experimentally, while the models where the $\mathrm{OH}$ groups were placed on $\mathrm{Al}$ that shared a double four ring (D4R) were also disfavoured.

Another example of anion disorder can be seen for AlPO-CJ2 (nominal formula $\left.\left(\mathrm{NH}_{4}\right)_{0.89}\left(\mathrm{H}_{3} \mathrm{O}\right)_{0.11}\left(\mathrm{AlPO}_{4}\right)(\mathrm{OH})_{0.33}(\mathrm{~F})_{0.67}\right)$, which has been studied in detail by Taulelle and coworkers by multinuclear NMR spectroscopy.[175-178] The crystal structure contains two anion sites (bridging $\mathrm{Al}^{\mathrm{V}}-\mathrm{X}-\mathrm{Al}^{\mathrm{VI}}$ and terminal $\mathrm{Al}^{\mathrm{VI}}-\mathrm{X}$ ) that were believed to be randomly occupied by either $\mathrm{OH}^{-}$or $\mathrm{F}^{-} .{ }^{19} \mathrm{~F}$ NMR confirmed that the fluoride occupies the bridging and terminal anion sites, and the presence of signals at -121 and -124 ppm was attributed to terminal $\mathrm{F}^{-}$adjacent to bridging $\mathrm{OH}^{-}$or $\mathrm{F}^{-}$, respectively, with the relative intensities indicating that the $\mathrm{Al}^{\mathrm{V}}-\mathrm{OH}-\mathrm{Al}^{\mathrm{VI}}-\mathrm{F}$ motif is most prevalent.[177,178] Detailed highresolution homo- and heteronuclear correlation spectra provided further insight and allowed the authors to suggest that the synthesis of AlPO-CJ2 starts with the formation of four-membered units, which condense with the random loss of $\mathrm{OH}^{-}$or $\mathrm{F}^{-}$to give a statistical distribution of bridging anions.[175-177] Taulelle and co-workers applied a similar approach to investigate the anion distribution in AlPO DNL-1 (-CLO framework type).[178] It was shown that $\mathrm{F}^{-}$could either reside in a D4R cage or was bound terminally to $\mathrm{Al}$ or $\mathrm{P}$ and, where terminal $\mathrm{F}^{-}$was present, this prevented occlusion of fluoride in the adjacent D4R. 
DNL-1 also highlights the use of "co-templating", where multiple organic species are present in solution during the synthesis of the material. It is often unclear from crystallography whether these species are all included in the as-made material. DNL-1 is synthesised in the presence of 1-ethyl,3-methylimidazolium (emim) and 1,6diaminohexane (hda), both of which are incorporated into the structure, as confirmed by ${ }^{13} \mathrm{C}$ and ${ }^{15} \mathrm{~N}$ NMR spectroscopy.[178] Two well-defined and magnetically-distinct locations were observed for the emim (in the lta-type pores and at channel intersections), while the hda was located only within the lta-type pores, but in a random orientation. In contrast, for STA-15, although a co-templating effect is observed (i.e., crystallisation is increased when tetraphenylphosphonium and an azaoxacryptand are added to the tetrapropylammonium used as an SDA), ${ }^{13} \mathrm{C}$ NMR clearly shows that the co-templates are not included into the final product.[179]

Decomposition of the organic species under the synthesis conditions can often lead to AlPOs containing unexpected SDAs, sometimes as a mixture of species. For example, the decomposition of dialkylformamide solvents often leads to the exclusive incorporation of the dialkylammonium species in the final AlPO.[180] Kongshaug et al.[181] used ${ }^{13} \mathrm{C}$ NMR to demonstrate that, while tetramethylammonium is the dominant SDA in UiO-12 (giving an intense resonance at $36.3 \mathrm{ppm}$ ), a minor resonance at $28.1 \mathrm{ppm}$ is indicative of the presence of $\sim 35 \mathrm{~mol} \%$ methylamine. Rather than decomposition of the solvent, Griffin et al. observed that, during ionothermal synthesis of CHA-type SIZ-4, the 1-alkyl,3methylimidazolium cations could undergo a transalkylation reaction.[182] When 1butyl,3-methylimidazolium (bmim) is used as the ionic liquid, the 1,3-dibutylimidazolium product is too large to fit within the CHA cage and an ordered material containing only 1,3-dimethylimidazolium (dmim) forms. However, when the ionic liquid is 1-ethyl,3methylimidazolium, both the initial ion and the 1-ethyl,3-methylimidazolium (emim) product can be incorporated into the cages and a disordered material (as evidenced by multiple resonances in the ${ }^{13} \mathrm{C}$ NMR spectrum shown in Figure 18(c)) is formed. If the counterion is changed (from bromide) to the more nucleophilic chloride, only dmim is then detected within the pores using ${ }^{13} \mathrm{C}$ NMR spectroscopy. 
In addition to the static disorder discussed above, AlPOs may exhibit temporal disorder, particularly motion of the SDA or other guest molecules. One of the earliest investigations of guest dynamics in AlPOs was the motion of $\mathrm{D}_{2} \mathrm{O}$ in the so-called "triple helix" of water in VPI-5 (VFI-type framework). By comparing simulated and experimental ${ }^{2} \mathrm{H}$ NMR spectra, Goldfarb et al.[183] proposed a six-site exchange model, which could explain the experimental lineshapes at $244 \mathrm{~K}$ and at 275-337 K. However, between 244 and $275 \mathrm{~K}$ poor agreement was observed. Duer et al.,[184] demonstrated that, by coupling the Goldfarb model with a $\mathrm{C}_{2}$ rotation of the Al-bound water molecule, excellent agreement with experiment was achieved. Variable-temperature ${ }^{2} \mathrm{H}$ NMR spectroscopy has also been used to study dynamics of a range of other guest molecules within AlPOs.[185,186] Antonijevic et al.,[66] recorded variable-temperature ${ }^{27} \mathrm{Al}$ MQMAS and STMAS spectra of AlPO-14 containing isopropylammonium or piperidinium. The $\mu$ s-timescale dynamics of the SDA led to a temperature-dependent broadening of the satellite transitions and, hence, a broadening of the isotropic dimension of the STMAS spectrum but not the MQMAS spectrum.

On a slower timescale (typically hours to days), calcined AlPOs are hygroscopic. While the crystal structures of a few calcined, hydrated AlPOs have been determined, including two hydrated forms of CHA-type AlPO-34 containing either 11 of 12 water molecules per unit cell, $[187,188]$ it is generally difficult to identify the positions of water molecules within the framework, owing to their mobility. As shown in Figure 18(d), even when left on the bench exposed to ambient moisture, a sample of calcined AlPO-34 hydrates overnight and then exists as a dynamic mixture of the two possible hydrated structures in varying proportions. Ashbrook et al.[189] used ${ }^{31} \mathrm{P}$ and ${ }^{27} \mathrm{Al} \mathrm{NMR}$ to demonstrate that the as-made AEN-type AlPO, JDF-2, was also hygroscopic, converting to AlPO-53(A) on exposure to ambient moisture on the timescale of weeks. Although the two structures have the same framework type and SDA (methylamine), JDF-2 has the space group Pcab, whereas the water in AlPO-53(A) lowers the symmetry to $P 2_{1} 2_{1} 2_{1}$, doubling 
the number of crystallographically-distinct $\mathrm{P}$ and $\mathrm{Al}$ sites. The transformation could readily be followed by ${ }^{31} \mathrm{P}$ MAS and ${ }^{27} \mathrm{Al}$ MQMAS NMR.

When AlPOs are doped to give SAPOs $\left(\mathrm{Si}^{4+}\right.$ substitution) or MAPOs, (substitution of divalent metal cations, $\mathrm{M}^{2+}$ ), the compositional disorder and additional requirements for charge balancing (by, e.g., anion vacancies or Brønsted acid site formation) leads to more complicated NMR spectra.[152,161,190] As it is challenging to obtain information on the spatial arrangement of Si in SAPO frameworks using crystallographic techniques, much of the evidence for the proposed substitution mechanisms comes from either ${ }^{29} \mathrm{Si} \mathrm{NMR}$ spectroscopy or theoretical calculations. Substitution is expected to occur at a P site (as substitution on an $\mathrm{Al}$ site would lead to four disfavoured P-O-Si linkages), although the combined substitution of $\mathrm{Al}$ and $\mathrm{P}$, forming so-called "silica islands" can avoid such unfavourable linkages. ${ }^{29} \mathrm{Si}$ MAS NMR allows the identification and quantification of $\mathrm{Si}(4 \mathrm{Al})$ species (isolated substitutions) and $\mathrm{Si}(3 \mathrm{Al}), \mathrm{Si}(2 \mathrm{Al}), \mathrm{Si}(1 \mathrm{Al})$ and $\mathrm{Si}(0 \mathrm{Al})$ species, which are evidence of silica islands.[190,191] For example, the presence of silica islands has been investigated in the chabazite-type SAPOs, SAPO-34 and CAL-1, which have different Si content.[192,193] As shown in Figure 19(a), spectra for the materials with higher Si content are dominated by $\mathrm{Si}(4 \mathrm{Al})$, but resonances for $\mathrm{Si}(3 \mathrm{Al}), \mathrm{Si}(2 \mathrm{Al}), \mathrm{Si}(1 \mathrm{Al})$ and $\mathrm{Si}(0 \mathrm{Al})$ are also present. At lower Si content, only the $\mathrm{Si}(4 \mathrm{Al}), \mathrm{Si}(3 \mathrm{Al})$ and $\mathrm{Si}(2 \mathrm{Al})$ resonances are present, indicating that a smaller number of smaller silica islands are present. The ordering observed for Si substitution depends to some extent on the framework structure, but also on the synthetic approach used.

For MAPOs, the mechanism of substitution is straightforward, with $\mathrm{M}^{2+}$ replacing $\mathrm{Al}^{3+}$, resulting in ${ }^{31} \mathrm{P}$ NMR spectroscopy being the ideal tool to probe the occupancy of the neighbouring $\mathrm{Al} / \mathrm{M}$ sites, as the ${ }^{31} \mathrm{P}$ NMR parameters vary with the number of $\mathrm{P}-\mathrm{O}-\mathrm{M}$ linkages. For example, Philippou et al.[194] carried out a ${ }^{31} \mathrm{P}$ magic-angle turning (MAT) NMR experiment (shown in Figure 19(b)) for SOD-type MgAPO-20 and showed a systematic shift of $c a .+6$ ppm per additional NNN Mg. The ${ }^{31} \mathrm{P}$ CSA also varied, with the increased span reflecting the more distorted local geometry, and the variation in skew 
reflecting the change in point symmetry at the $\mathrm{P}$ site. Seymour et al. recently carried out a detailed experimental and computational study of the Mg and Zn substitution in STA-2 and observed a similar trend in ${ }^{31} \mathrm{P}$ isotropic shift for both materials.[81] ${ }^{1} \mathrm{H}$ and ${ }^{27} \mathrm{Al} \mathrm{NMR}$ spectra showed that loss of bridging $\mathrm{OH}^{-}$was the charge-balancing mechanism, and indicated preferential substitution of $\mathrm{Mg}^{2+}$ for Al1 (located in single 6-membered rings) rather than A12 (located in D6Rs). Most other MAPOs include (nominally divalent) transition metal cations, generally chosen for their favourable catalytic behaviour. The presence of unpaired electrons and potentially variable oxidation states of these species make solid-state NMR spectroscopy more challenging. The first reported NMR experiments on a paramagnetic MAPO were by Canesson and Tuel,[195] who recorded the ${ }^{31} \mathrm{P}$ NMR spectra of CoAPO-50. A single resonance was observed at $-30 \mathrm{ppm}$ in the ${ }^{31} \mathrm{P}$ MAS NMR spectrum, arising from $\mathrm{P}(\mathrm{OAl})_{4}$ sites, but with intense spinning sidebands typical of a large paramagnetic shift anisotropy and indicating a pseudocontact interaction with $\mathrm{Co}^{2+}$. The static ${ }^{31} \mathrm{P}$ spectrum revealed an additional, very broad resonance, from around 0 to 9000 ppm with a maximum at 2520 ppm, corresponding to $P$ with at least one $\mathrm{Co}^{2+} \mathrm{NNN}$, experiencing a large through-bond hyperfine interaction. The authors recorded a ${ }^{31} \mathrm{P}$ shift of $\sim 9500 \mathrm{ppm}$ for $\mathrm{P}(\mathrm{OCo})_{4}$ in the cobaltophosphate, DAF-2 and, therefore, assumed that each $\mathrm{P}-\mathrm{O}-\mathrm{Co}^{2+}$ linkage contributed around 2000 to $3000 \mathrm{ppm}$ to the position of the peak maximum. More recently, Mali et al.[196] studied a series of CHA-type MAPOs containing $\mathrm{Ni}, \mathrm{Co}, \mathrm{Fe}$ and $\mathrm{Mn}$ and showed that a similar broad resonance was present in the static ${ }^{31} \mathrm{P}$ NMR spectra of all materials apart from the as-made MnAPO (where the absence of the resonance was attributed to very rapid relaxation rendering it invisible, as the ${ }^{31} \mathrm{P}$ NMR spectrum of the calcined MnAPO did exhibit a very broad resonance).

Gallophosphates (GaPOs) can also form many zeolitic framework types. However, their lower thermal stability and the high cost of Ga has resulted in less intense investigation of these materials. Solid-state NMR spectroscopy has been used both to confirm the structures of GaPOs and to investigate disorder within them. Examples include the identification of $\mathrm{P}(\mathrm{OGa})_{3}(\mathrm{OH})$ and $\mathrm{P}(\mathrm{OGa})_{3}(=\mathrm{O})$ species in the interrupted 
(incompletely condensed) framework of $\mathrm{GaPO}_{4}$ cloverite using ${ }^{31} \mathrm{P}$ MAS NMR[197] and investigation of the collapse of calcined, hydrated CHA-type GaPO-34 upon heating.[198]

Metal-organic frameworks (MOFs) comprise of inorganic nodes (typically metal cations or metal oxide/carboxylate-based clusters) connected in an infinite microporous framework by organic linkers (typically based on rigid aromatic molecules such as benzene-1,4-dicarboxylate (bdc) and benzene-1,3,5-tricarboxylate (btc)). In addition to displaying great structural flexibility, depending on the connectivities of the nodes and linkers chosen, there is often wide compositional flexibility for a given structure, with the same framework type forming with several different metals and/or linkers and the possibility of combining multiple metals or linkers into the same structure. For more information on the use of solid-state NMR for the characterisation of MOFs see Refs. $[152,160]$.

In MOFs that exhibit isoreticularity, the same framework connectivity can form with linkers with different chemical functionality or physical size, allowing tuning of both the pore chemistry and volume.[151] MOFs can also be prepared with mixed linkers, allowing a further tuning of their properties. Kong et al.[199] recently probed the distribution of linkers in mixed-linker versions of MOF-5 using ${ }^{13} \mathrm{C}-{ }^{15} \mathrm{~N}$ REDOR experiments to measure the mean distances between pairs of isotopically-labelled spins. As shown in Figure 20(a), comparison to MD calculations carried out assuming different ordering behaviour (e.g., large clusters, small clusters, random distribution or alternating linkers), the linker distribution present could be determined. The most complex system investigated with the authors contained three linkers, all of which were shown to be in alternation. The structures generated by MD also allowed the calculation of theoretical $\mathrm{CO}_{2}$ adsorption isotherms for the MOFs, which confirmed the ordering determined by the NMR experiments, demonstrating the link between the atomic-scale distribution of the linkers and the bulk properties of the material. Jayachandrababu et al.[200] used highresolution ${ }^{1} \mathrm{H}$ spin-exchange experiments, combined with $\mathrm{MD}$, to probe the short-range 
ordering within a series of zeolitic imidazolate frameworks (ZIFs), demonstrating an intimate mixing of the linkers within the MOFs (rather than clustering behaviour).

It is also possible to prepare MOFs with mixed metals, again providing fine tuning of the properties of the material. The NMR parameters of metal centres in MOFs have been shown to be sensitive to the structure of the materials[152,153,201] and these can provide information on the cation distribution in mixed-metal MOFs. However, in mixed Sc/Al MIL-100, Mitchell et al. were unable to unambiguously determine whether the individual trimeric metal nodes contained mixtures of Sc and $\mathrm{Al}$, owing to the additional $\mathrm{OH} / \mathrm{H}_{2} \mathrm{O}$ disorder present within the coordination sphere of the metal centres.[202] This latter disorder was shown to dominate the ${ }^{45}$ Sc NMR parameters. Gul-E-Noor et al. used ${ }^{1} \mathrm{H}$ and ${ }^{13} \mathrm{C}$ MAS NMR spectra to characterise the Cu/Zn HKUST-1 series, where the presence of the paramagnetic $\mathrm{Cu}^{2+}$ and diamagnetic $\mathrm{Zn}^{2+}$ led to more distinct shift differences.[203] Some MOFs also contain mixed anionic species, which often cannot be distinguished by crystallographic techniques, but can readily be studied by NMR spectroscopy. For example, Cadiau et al.[204] used quantitative ${ }^{1} \mathrm{H}$ and ${ }^{19} \mathrm{~F}$ NMR spectroscopy to characterise $\mathrm{OH}^{-} / \mathrm{F}^{-}$disorder in $\mathrm{CuAlF}_{4.5}(\mathrm{OH})_{0.5}\left(\mathrm{H}_{2} \mathrm{O}\right)[\mathrm{HAmTAZ}]_{2}$ (HAmTAZ = 3-amino-1,2,4-triazole). Owing to the paramagnetic $\mathrm{Cu}^{2+}$ centres, integration over the intense spinning sidebands was required to achieve full quantification, after which it was clear that the relative intensity of the ${ }^{19} \mathrm{~F}$ resonance corresponding to Al-F-Cu species was lower than expected, indicating that some $\mathrm{Al}-\mathrm{OH}-\mathrm{Cu}$ bridges must also occur. He et al.[205] recorded the ${ }^{17} \mathrm{O}$ NMR spectra of enriched MOFs and showed that, for UiO-66 $\left(\mathrm{Zr}_{6} \mathrm{O}_{4}(\mathrm{OH})_{4}(\mathrm{bdc})_{6}\right)$, the three types of $\mathrm{O}$ site present $\left(\mu_{3}-\mathrm{OH}, \mu_{3}-\mathrm{O}\right.$ and $\left.-\mathrm{CO}_{2}\right)$ had very different chemical shifts $(65,386$ and 278 ppm, respectively) and quadrupolar coupling parameters $(6.5,2.0$ and $7.1 \mathrm{MHz}$, respectively), allowing the very clear identification of the two types of anion in the inorganic $\mathrm{Zr}_{6} \mathrm{O}_{4}(\mathrm{OH})_{4}$ units.

In addition to preparing MOFs directly with a given chemical functionality, postsynthetic modification can be used to introduce new active sites after a MOF has been prepared.[206] Since the modifications are rarely periodic, solid-state NMR spectroscopy 
is commonly used to confirm that the modification has taken place and, in some cases, to quantify the extent of the transformation. For example, Wittmann et al.[207] modified $\mathrm{NH}_{2}$-functionalised MIL-101 with phenylisocyanate, to give pendant phenylurea groups, which improved the water stability of the MOF. As shown in Figure 20(b), ${ }^{15} \mathrm{~N}$ NMR spectroscopy confirmed that all $\mathrm{NH}_{3}$ groups had been converted to the urea linkage. Despite a marginally higher water uptake for the modified MOF, ${ }^{1} \mathrm{H}^{27} \mathrm{Al}$ dipolar $\mathrm{HMQC}$ correlation spectra (Figure 20(c)) were able to demonstrate that there was less water spatially near the $\mathrm{Al}$ centres.

The linkers in many MOFs exhibit dynamic disorder: particularly bdc, which can undergo $C_{2}$ flipping, dabco (1,4-diazabicyclo[2.2.2]octane), which can undergo $C_{3}$ rotation, and imidazolates, which can undergo wagging and rocking motions.[151] Variabletemperature ${ }^{2} \mathrm{H}$ NMR spectroscopy of MOFs prepared with deuterated linkers is used regularly to investigate this motion in diamagnetic MOFs (a typical set of spectra, recorded for $\mathrm{Sc}_{2}(\mathrm{bdc})_{3}[208]$ is shown in Figure 21(a)). Comparison to simulation shows that the experimental spectra contain signals for the two distinct linkers in this MOF, but only one of which is undergoing a $180^{\circ}$ flip of the benzene ring. However, fewer studies have been carried out in paramagnetic MOFs owing to the additional practical challenges to spectral acquisition. Kolokolov et al.[209] studied the motion of the benzene rings in paramagnetic MIL-53(Cr) and MIL-47(V), showing that the lineshapes were a combination of the usual quadrupolar broadening and a large paramagnetic shift anisotropy for the MIL-53(Cr), but that the PSA contribution was much smaller for the MIL-47(V). In addition to linker rotation, the flexibility of MOFs also can result in motion of the entire framework. This change in framework configuration, often termed "breathing", may lead to a new crystalline phase (as is the case with MIL-53, which has an extremely complex conformational landscape[210,211]) or, in some cases, to a more disordered phase. For example, the dehydration of STAM-1 ( $\mathrm{Cu}(\mathrm{mmbtc}), \mathrm{mmbtc}=$ monomethyl ester of btc) leads to a lowering of the overall symmetry of the material and loss of long-range order, as evidenced by both splitting and broadening of the resonances in the ${ }^{13} \mathrm{C} N M R$ spectrum.[212,213] 
Solid-state NMR spectroscopy has been extensively applied to characterise guest molecules in MOFs, particularly where these exhibit spatial or temporal disorder and are, therefore, difficult to observe by crystallographic techniques.[152,153,214] In MOFs containing open metal sites, guest molecules are often bound to these and variation in the ${ }^{2} \mathrm{H},{ }^{13} \mathrm{C}$ or ${ }^{17} \mathrm{O}$ anisotropic NMR parameters with temperature can be used to determine the type and rate of restricted motion occurring, giving information on the binding strength and mode.[215-219] One of the systems studied in most detail to date is $\mathrm{CO}_{2}$ adsorbed in Mg-CPO-27 ( $\mathrm{Mg}_{2}($ dobdc $)$, dobdc = 2,5-dioxobenzene-1,4-dicarboxylate). Kong et al.[215] used the CSA of ${ }^{13} \mathrm{CO}_{2}$ to investigate this binding, showing that the $\mathrm{CO}_{2}$ molecule rotated about the $\mathrm{Mg}-\mathrm{O}$ bond axis at an angle, $\theta$, which varied from $69^{\circ}$ at $210 \mathrm{~K}$ to $56^{\circ}$ at $375 \mathrm{~K}$, as shown in Figure 21(b). However, this model was subsequently revised by Wang et al.,[216] who used the ${ }^{17} \mathrm{O}$ NMR spectrum of $\mathrm{C}^{17} \mathrm{O}_{2}$ to show that, in addition to the rotation about the Mg-O axis, a local "wobbling" occurred about the molecular axis at an angle, $\alpha$, as well as a six-site exchange between equivalent binding sites (Figure 21(c)). Chemisorption of $\mathrm{CO}_{2}$ to give the formation of carbamates (reaction with $\mathrm{NH}_{2}$ functionality)[218] or carbonates (reaction with $\mathrm{OH}$ functionality)[219] has also been confirmed by solid-state ${ }^{13} \mathrm{C}$ NMR. As discussed above for linker motion, static ${ }^{2} \mathrm{H}$ NMR spectroscopy can also be used to provide detailed information on the motion of guests within MOFs.

Wholly-organic crystalline microporous frameworks are a relatively new class of materials and the application of NMR spectroscopy to their characterisation has been almost exclusively to confirm an ordered structure. However, a recent study by Blanc et al.[220] has shown the potential of DNP-assisted ${ }^{13} \mathrm{C}$ and ${ }^{15} \mathrm{~N}$ NMR spectroscopy (at natural abundance) to provide detailed structural information for a series of microporous organic polymers (MOPs) with mixed organic functionality.

\subsection{Molecular Systems}

It might be thought that structural characterisation of molecular solids is an easier prospect than that of the extended systems discussed above, as information on the 
structure of the molecule itself is available from techniques such as solution-state NMR spectroscopy or mass spectrometry and, in many cases, single-crystal XRD experiments are also feasible. However, systems with significant disorder or dynamics, materials where single crystals can't be grown and the accurate determination of $\mathrm{H}$ positions can still pose a significant challenge. NMR has been widely used to study the rich polymorphism that is often found for molecular systems, as the chemical shift is a very sensitive probe of the local environment (including both the covalent bonds that are formed and the spatial proximity of other nuclei).[221-226] As such, this can provide information not just on molecular structure (as in solution-state NMR) but also on the spatial arrangement of molecules in the solid state (i.e., the crystal packing), and the number of distinct molecules within a structure. Figure 22a shows ${ }^{13} \mathrm{C}$ CP MAS spectra of two forms of finasteride.[227] In Form I, only one signal is observed for each carbon in the molecule, confirming just one molecule is present in the asymmetric unit. However, the spectrum for Form II is not only clearly different (e.g., the change in chemical shift for carbon 1) but the splitting of each resonance reveals there are two distinct molecules in the structure. In recent work, Harris and co-workers have demonstrated how NMR spectroscopy can be used to follow crystallisation of small molecules (and to identify the formation of unusual or new polymorphs during this process) using an in situ approach termed CLASSIC (Combined Liquid- And Solid-State In situ Crystallization) NMR.[228-232] In this method, a homogenous solution is initially prepared inside a sealed insert within a rotating NMR rotor at high temperature. Crystallization is induced by a rapid decrease in temperature, forming a supersaturated solution, and can be followed by the interleaved acquisition of alternating solution-state (direct acquisition) and solid-state (using CP) NMR spectra. For glycine, $[231,232]$ the in situ ${ }^{13} \mathrm{C}$ spectra of ${ }^{13} \mathrm{C}$-enriched molecules showed the formation of the $\alpha$ polymorph from an aqueous solution, whereas crystallization from deuterated water initially formed the $\alpha$ polymorph (in $1.5 \mathrm{~h}$ ), but this then transformed to the $\gamma$ polymorph at longer times. The $\beta$ polymorph could be formed by in situ crystallization from a methanol/water solution, and transformed to the $\alpha$ polymorph over longer times. 
In addition to the chemical shift, interactions between spins can also inform on the three-dimensional arrangement of molecules in the solid. It is possible to measure dipolar couplings (and, therefore, corresponding distances or spatial proximities) using techniques such as REDOR and two-dimensional correlation spectra.[2,3] In solution, J couplings are exclusively intramolecular interactions, but in solids they can also provide information on the hydrogen-bonding motifs that direct molecular packing. As an example, Figure $22 \mathrm{~b}$ shows two-dimensional ${ }^{15} \mathrm{~N}$ homonuclear correlation spectra of deoxyguanosine derivatives.[226,233] The pairs of peaks either side of the diagonal indicate an interaction between two nuclei, and while most signals result from intramolecular interactions, the correlations highlighted (N1-N7 and N2-N7) provide information on the intermolecular hydrogen bonds present and the self-assembly of the molecules into ribbons or quartets. More recently, intermolecular J couplings have been observed between chalcogen-containing aromatic molecules.[234,235] Although often termed "through-space" J couplings, these interactions are between two atoms that lie within or close to the sum of their van der Waals radii, leading to significant electron density between them, although a formal covalent bond would not be drawn. The ${ }^{77}$ Se MAS NMR spectrum of Naphtho[1,8-cd]1,2-diselenole tertbutylphosphine exhibited two different couplings to ${ }^{31} \mathrm{P}$ (of similar magnitude, $\sim 320-340 \mathrm{~Hz}$ ); one conventional throughbond coupling to the adjacent ${ }^{31} \mathrm{P}$ atoms, and one intermolecular coupling to ${ }^{31} \mathrm{P}$ in the next molecule (a distance of $3.5 \AA$ ). [234] Perhaps of more interest in the context of disorder, was the observation of a ${ }^{31} \mathrm{P} /{ }^{31} \mathrm{P}$ J coupling $(88 \mathrm{~Hz})$ between crystallographically-equivalent $\mathrm{P}$ species in two separate molecules. Interestingly, in a fraction of the molecules $(27 \%)$ the expected magnetic equivalence of the two $\mathrm{P}$ species was lifted by an intramolecular heteronuclear coupling to ${ }^{77} \mathrm{Se}$, reflecting an "isotopic disorder" of the only NMR-active isotope $\left({ }^{77} \mathrm{Se}, 7.6 \%\right) \cdot[235]$

In many cases, the structures of polymorphs (and particularly the $\mathrm{H}$ positions and any hydrogen-bonding networks) are solved or refined using an NMR crystallographic approach, where DFT calculations (on proposed or partial structural models produced by diffraction) are combined with NMR spectroscopy. For example, Kibalchenko et al.,[236] 
studied three possible models for the hydrogen-bonding network in $\alpha$-D-galactose. Upon computational optimization, two models were indistinguishable, but calculation of the ${ }^{1} \mathrm{H}$ NMR parameters were able to determine which of the remaining two options matched best with experiment. In some cases, where structural models are unavailable or incomplete, it is possible to use computational crystal structure prediction (CSP)[11,237239] to determine the structure of one or more polymorphic forms of a solid. This typically involves a conformational search for an isolated molecule using molecular mechanics $(\mathrm{MM})$, followed by the generation of a range of candidate structures using Monte Carlo techniques. These structures are then optimized computationally, before the lower-energy structures are selected as possible solutions and calculated parameters compared to experiment. The AIRSS[91,92] approach (discussed above) can also be used in a similar manner.

Although many polymorphs of pure samples themselves exhibit an ordered structure, many real samples can contain mixtures of polymorphs, or pseudopolymorphs (i.e., crystalline solid adducts containing solvent molecules within the structure, in either stoichiometric or non-stoichiometric proportions).[221,223,224] NMR can be used not only to detect the proportion of polymorphs present but also the tautomeric forms of molecules, e.g., whether neutral molecules or zwitterions are seen. Resonances associated with the included solvents can be observed directly in the NMR spectrum, as also can changes in some of the chemical shifts of the molecule of interest, as a result of the variation in crystal packing or the different intermolecular interactions experienced. If spectra are sufficiently quantitative, the host/solvent ratio can also be determined.[221,223] For finasteride, discussed above, solvates are formed with acetic acid, ethyl acetate, diethylether, dioxane, isopropylalcohol and tetrahydrofuran. With the exception of acetic acid, all were found to be hemihydrates (with a finasteride:solvent ratio of 2:1), and to have a common structure.[227] Many pharmaceutical formulations are composed of complex mixtures that contain both the active pharmaceutical ingredient(s) (or APIs) and a number of excipients, including starch, talc, stearates, etc., to vary the physical and chemical properties of the material, many of which may also be less well 
crystalline. NMR spectroscopy has been used to characterise the number and type of species present in these samples, as shown in Figure 22c for a simulated drug formulation containing 5\% bambuterol hydrochloride (BHC),[221,223] with a number of studies devoted to developing protocols (often using internal standards) for quantification of the various crystalline and amorphous species present.[220,221,223,240] This was illustrated recently by Caldarelli and co-workers for Meprobamate, an anxiolytic agent.[241] To overcome some of the problems associated with low concentrations of APIs in tablet formulations it can be possible to utilise some of the less prevalent nuclei in these molecules, such as ${ }^{19} \mathrm{~F}$, as demonstrated by Brus et al., for atorvastatin,[242] where only signal from the drug is then observed.

A pure molecular solid may exhibit some disorder, often in the position or orientation of a functional group on the molecule or of included solvent. This will lead to a variation in chemical shifts and, therefore, a line broadening. In some cases, resolution of all peaks and the extraction of structural information is still possible, although as the extent of disorder increases this can become increasingly difficult. For amorphous materials, peaks can be very broad, limiting the detailed information available, although chemically-different species may still be able to be distinguished. When samples are prepared by flash-freezing of liquids, a range of molecular conformations may be present in a solid, leading to linebroadening, while some processing (e.g., grinding) can induce recrystallization.[221,223] This can be easily followed using NMR, as shown by Apperley et al., where ${ }^{13} \mathrm{C}$ CP MAS spectra showed crystallisation of amorphous nifedipine upon grinding, while indomethacin remained amorphous when subjected to the same process.[243] Recent work by Cadars et al. used computation to reproduce the variation in chemical shift that can occur from slight structural disorder (i.e., small conformational variations) in a molecular solid.[244] DFT calculations of the low-energy vibrational modes in bisphosphoamine were used to provide a set of physically reasonable local distortions, used to represent a static distribution of local geometries. ${ }^{31} \mathrm{P}$ NMR parameters were calculated and used to simulate two-dimensional correlation spectra to identify the structures compatible with the local disorder (i.e., matching the shape of the cross peaks 
observed, as discussed in Section 4). Emsley and co-workers[245] showed how highresolution spectra could be obtained from similar two-dimensional spectra of disordered solids. Using the correlation of the disorder in ${ }^{13} \mathrm{C}$ and ${ }^{31} \mathrm{P}$ shifts in $N, N$-bis(diphenylphosphino)-N-((S)-R-methylbenzyl)amine, they were able to obtain a sheared projection of a two-dimensional heteronuclear correlation spectrum that is free of any inhomogenous broadening (although this occurs in both direct and indirect spectral dimensions). The increased resolution enabled the 8 sites in the structure to be resolved and the correlations between them to be determined.

In many cases, disorder in the position of solvent molecules causes the disorder seen in NMR spectra of molecular solids. In recent work on Naproxen (a non-steroidal anti inflammatory), Bond et al.[246] combined XRD and NMR to study the structure of the tetrahydrate. Diffraction showed an average structure with space group C2, containing layers of ordered naproxen molecules, with ${ }^{13} \mathrm{C}$ MAS NMR also showing two possible orientations of the carboxyl group. The $\mathrm{Na}^{+} / \mathrm{H}_{2} \mathrm{O}$ regions show complex disorder, but the single peak in the ${ }^{23} \mathrm{Na}$ MAS NMR spectrum confirmed the local symmetry in these regions.[246] In some solids, the deviation from true periodicity can be caused by differences in the stacking of molecules (particularly for aromatic systems, where crystal packing can be determined by favourable $\pi$ - $\pi$ stacking interactions). This is the case for the molecules shown in Figure 23a, a hydroxyboroxophenanthrene (that forms a dimer in the solid state) and the corresponding anhydride.[87] The ${ }^{13} \mathrm{C}$ spectrum of the anhydride shown in Figure 23b contains broadened lineshapes thought to result from a disordering of the orientation of the B-O-B bridges in the $\pi$ stacks. This was confirmed using DFT calculations for a series of models with different stacking patterns, and the prediction of the variation in chemical shifts that these would produce. ${ }^{11} \mathrm{~B}$ MQMAS spectra were able to detect the solid-state transformation of the dimer to the anhydride (a reaction not observed by diffraction) upon gentle heating, by the appearance of a second resonance (shown by the green line in Figure 23c). A mechanism for this reaction within the solid state was suggested by computation, as shown in Figure 23d, where reaction of two halves 
of two different stacked dimers can produce the anhydride with little effect on the surrounding structure.

For many molecular solids temporal disorder (i.e., dynamics) is also an important concern. As described in Section 4, the effect of dynamics on an NMR spectrum depends on the timescale of the motional process, and on the magnitude of the interactions that affect a spectrum. Variable-temperature NMR spectra reveal the presence of dynamics, either through line broadening, coalescence or changes in anisotropic lineshapes. By comparison to simulation it is possible to extract information on types and rates of motion, with Arrhenius-like plots providing activation energies. Relaxation measurements (as a function of temperature) can also provide insight into dynamic processes. These types of experiment have all been used to great effect on molecular solids. Recent examples include ${ }^{2} \mathrm{H}$ NMR of furosemide-isonicotinamide co-crystals, demonstrating fast exchange of the $\mathrm{NH}_{2}$ protons and rotation of the sulphonamide group around the C-S bond,[247] the use of EXSY experiments and ${ }^{13} \mathrm{C} \mathrm{T}_{1}$ relaxation measurements of formoterol fumerate to show fumarate motion (on a frequency of 100s of $\mathrm{MHz}$ ) with an activation energy of $32 \mathrm{~kJ} \mathrm{~mol}^{-}$ ${ }^{1}$, [248] and variable-temperature ${ }^{17} \mathrm{O}$ NMR of sulfonic acids, demonstrating a three-fold rotational jump of the $\mathrm{SO}_{3}^{-}$groups (with an activation energy of $\sim 48 \mathrm{~kJ} \mathrm{~mol}^{-1}$ ).[249] Earlier work by Cutajar et al.,[250] demonstrated the linebroadening effect of microsecond timescale motion on the ${ }^{2} \mathrm{H}$ MAS spectrum of oxalic acid. There are two ${ }^{2} \mathrm{H}$ sites in the structure, i.e., $\mathrm{CO}_{2}{ }^{2} \mathrm{H}$ and ${ }^{2} \mathrm{H}_{2} \mathrm{O}$ species, with the latter exhibiting broader line owing to rotation around the $\mathrm{C}_{2}$ axis modifying the ${ }^{2} \mathrm{H}$ quadrupolar interaction experienced. The origin of the broadening (i.e., as a result of dynamics and not a distribution of chemical shifts) was confirmed using two-dimensional double-quantum experiments, as the $\mathrm{m}_{\mathrm{I}}=+1$ $\leftrightarrow \mathrm{m}_{\mathrm{I}}=-1$ transition is unaffected by the quadrupolar interaction and so does not exhibit any motional broadening (but would be affected by a distribution of shifts). In a very elegant study, Hodgkinson and co-workers[251] combined XRD, NMR spectroscopy and MD simulations to investigate dynamic disorder of octafluoronapthalene $\left(\mathrm{C}_{10} \mathrm{~F}_{8}\right)$. Variabletemperature ${ }^{19} \mathrm{~F}$ relaxation measurements were able to identify two distinct motional processes (a fast process with a correlation time of ns at ambient temperature, and a much 
slower process on the $\mu$ s timescale). As shown in Figure 24a, MD simulations reveal the faster process to involve a jump of $\sim 40^{\circ}$ in molecular orientation (Figure $24 \mathrm{~b}$ ). The more extreme rotations also observed are thought to bring about full rotation of the molecules in the solid, and lead to the variations observed in the $\mathrm{T}_{1 \rho}$ relaxation rate.

\subsection{Biomaterials}

Biomaterials are inherently disordered systems, often containing multiple interacting organic and inorganic phases. The application of solid-state NMR spectroscopy to structural biology is a vast and growing field, far beyond the scope of this review. Here, we will focus only on a few recent highlights in the field, showcasing the rich atomic-scale detail available from NMR spectroscopy, even in complex materials. For brevity, we have restricted our discussion to bioinorganic materials and, for more comprehensive reviews, readers are directed to Refs. [153,252-254]

The precise microstructure of bone has been under scrutiny by solid-state NMR spectroscopy for years, with models based on apatite $\left(\mathrm{Ca}_{10}\left(\mathrm{PO}_{4}\right)_{6}(\mathrm{X})_{2}\right.$ where, for hydroxylapatite (HA) $\mathrm{X}=\mathrm{OH}$, for fluorapatite (FA) $\mathrm{X}=\mathrm{F}$ and for chlorapatite $\mathrm{X}=\mathrm{Cl}$ ), often substituted with $\mathrm{Mg}^{2+}, \mathrm{CO}_{3}{ }^{2-}, \mathrm{SiO}_{4}{ }^{4-}$, etc. to provide a more accurate model or to improve the properties of the material.[80,254-258] A great deal of detailed NMR experiments have been carried out for apatites, investigating the location of water molecules, the surface termination and the substitution of various anions and cations.[122] For example, Laurencin et al. used ${ }^{43}$ Ca NMR spectroscopy and DFT calculations to probe the substitution of $\mathrm{Mg}^{2+}$ into $\mathrm{HA}$, showing that the $\mathrm{Mg}^{2+}$ preferentially occupies the 7coordinate Ca2 site rather than the 9-coordinate Ca1.[80] However, work recently reported by Duer and co-workers has led to a step-change in the understanding of the inorganic component of bone.[259,260] Using a combination of detailed NMR spectroscopy, X-ray diffraction and computational modelling, the authors were able to confirm that the inorganic platelets found in bone are best modelled as very thin apatite-like layers sandwiched between octacalcium phosphate (OCP) and separated by hydrated octacalcium phosphate citrate layers as shown in Figure 25. This work follows on from 
early indications by techniques such as ${ }^{43} \mathrm{Ca}$ NMR spectroscopy that the spectral signature of natural bone did not agree well with the spectra recorded for model apatites.[261] Apatitic materials are also found in teeth, and solid-state NMR spectroscopy of natural samples of these tissues has revealed much useful and unsuspected information.[257,262] As an example, Laurencin et al. used multinuclear solid-state NMR spectroscopy, including ${ }^{23} \mathrm{Na}\left\{{ }^{31} \mathrm{P}\right\}$ REDOR experiments to confirm a close $\mathrm{Na}-\mathrm{P}$ proximity and provide direct evidence of sodium within apatite in natural samples of bones and teeth.[262]

The biomineralisation of silica occurs in a wide range of organisms from bacteria up to humans. One spectacular example are the intricate cell walls formed in diatoms (a type of algae), which are made from amorphous hydrated $\mathrm{SiO}_{2}$. The walls are produced from the intracellular condensation of $\mathrm{Si}(\mathrm{OH})_{4}$ species, which are stabilised during this process by the organic biomolecules. Diatoms have been studied using ${ }^{29} \mathrm{Si}$ NMR spectroscopy, both using conventional MAS and CP-based experiments.[263,264] In some cases, diatoms have been grown in artificial water containing ${ }^{29} \mathrm{Si}$ (either as ${ }^{29} \mathrm{SiO}_{2}$ or $\mathrm{Na}^{29} \mathrm{SiO}_{3}$ ), enabling enriched biominerals to be produced.[263,264] The condensation of the Si species was shown to vary little between different diatom species, with a $\mathrm{Q}^{4} / \mathrm{Q}^{3}$ ratio of $70-75 \%$, although this ratio differs slightly between the whole cell and the cell wall.[263] Isotopic enrichment also enables two-dimensional spectroscopy to be carried out, with ${ }^{1} \mathrm{H}-{ }^{29} \mathrm{Si}$ correlation experiments confirming the presence of $\mathrm{Q}^{3}$ species.[264] The organic components of diatoms can also be studied using solid-state NMR spectroscopy. One of the most detailed studies (using ${ }^{13} \mathrm{C}$ and ${ }^{15} \mathrm{~N}$ enriched diatoms) showed that the molecules associated with the silica matrix were glucose-based carbohydrates and lipids, which showed differing mobilities based on the degree of interaction they experienced.[264]

Calcium carbonate is also an important biomineral, which can be found in shells and pearls. Although a number of more ordered polymorphs (and pseudopolymorphs) exist, amorphous calcium carbonate is also found in nature. In some crustaceans, calcium is stored as amorphous calcium carbonate in a gastrolith. The inorganic component of lobster and crayfish gastroliths was shown (using ${ }^{13} \mathrm{C}$ and ${ }^{1} \mathrm{H}$ NMR spectroscopy) to 
contain hydrogen-rich carbonate ions, confirming this material is highly hydrated.[265,266] Two-dimensional ${ }^{31} \mathrm{P} /{ }^{13} \mathrm{C}$ NMR experiments, revealed that inorganic phosphate and phosphoenolpyruvate anions were widely dispersed within the amorphous material and played some role in the stabilization of the amorphous calcium carbonate in vivo, while measurements of the dipolar coupling determined ${ }^{31} \mathrm{P}_{-}{ }^{13} \mathrm{C}$ distances of 8-9 A..[265,266]

A recent approach to studying biomaterials in situ is whole-body high-resolution (HR) MAS. In this approach a small living organism can be placed in a MAS rotor and rotated relatively slowly $(\sim 2-3 \mathrm{kHz})$ to minimise injury and death. Conventional MAS NMR spectroscopy enables in vivo metabolic profiles to be obtained; an approach that has been applied to bacteria, C. elegans worms, or Drosophila melanogaster flies.[267-271] However, MAS NMR spectra of whole organisms can be very complex, and although some metabolites can be identified and quantified, some signals cannot be assigned owing to spectral overlap. While two-dimensional spectroscopy can sometimes improve resolution, the issue of where in the organism the relevant metabolites are located remains. Recently, work by Sarou-Kanian et al.,[272] has extended in vivo HR MAS studies by applying a magnetic field gradient along the MAS axis, enabling both chemical shift information and spatial localisation of metabolites to be determined. Using spatiallyresolved ${ }^{1} \mathrm{H}$ spectra they were able to produce a complete mapping of the metabolites in a live Drosophila fly, and demonstrated muscle degradation in the thorax by a decrease in $\beta$ alanine signals.

\subsection{Glasses and Cements}

In contrast to materials such as metal oxides, ceramics and minerals, that have structures containing a significant degree of periodicity, glasses are inherently amorphous, owing to the rapid cooling of molten materials during synthesis, typically preventing crystallization. This lack of long-range order causes glasses to exhibit an extensive array of desirable chemical, physical and mechanical properties (some of which are unique to this class of amorphous material), leading to their widespread implementation into a variety of 
technologies and consumer goods, including in optical devices, fuel cells, lasers, semiconductors and nuclear/toxic immobilisation materials. Although many aspects of the local structure in glasses are broadly conserved, for example the tetrahedral coordination of Si in silica-based glasses, the loss of long-range periodicity does result in variation of the exact local geometry, with distribution of bond lengths and bond angles observed throughout the glass network.[53,273-275] Clearly the lack of long-range order hampers the use of characterisation techniques that exploit the periodicity of solids, and has resulted in solid-state NMR spectroscopy establishing itself as an extremely valuable tool for the study of glasses.[53,273-275] Despite its fundamental advantage over diffraction-based techniques for amorphous solids, using solid-state NMR spectroscopy to investigate glass structure is by no means straightforward, as the variation in local geometry and the long-range disorder lead to spectra containing broad and overlapping resonances that are challenging to interpret. Recent work has demonstrated the value of first-principles calculations alongside experimental measurement in this regard, providing an ability to relate predicted or proposed geometries to spectral signatures.[276-78] Here, we highlight how solid-state NMR spectroscopy, in combination with other approaches, has been used to investigate the structure of glasses, a task of significant importance if the physical and chemical properties of these solids are to be understood and subsequently shaped to meet the technological demands of the future. Readers are referred to the more comprehensive reviews in Refs. [53,273-275] for more detail.

A brief search of the literature reveals that conventional one-dimensional MAS NMR spectroscopy has been used to great effect to characterise the local structure in glasses. As an example, in an attempt to verify a link between the nucleating ability of a glass and the local structure of the network-forming cations, Schneider et al.,[279] performed a detailed ${ }^{29} \mathrm{Si}$ MAS NMR study of metasilicate glasses (with compositions $\mathrm{Na}_{2} \mathrm{Ca}_{2} \mathrm{Si}_{3} \mathrm{O}_{9}, \mathrm{Na}_{4} \mathrm{CaSi}_{3} \mathrm{O}_{9}, \mathrm{CaSiO}_{3}$ and $\mathrm{CaMgSi}_{2} \mathrm{O}_{6}$ ) and isochemical crystals, chosen for the variation in their nucleation rates. Decomposition of the lineshapes in the ${ }^{29}$ Si MAS NMR spectra enabled the relative proportions of $\mathrm{Q}^{n}$ groups in each glass to be determined, with the $\mathrm{Q}^{2}$ environment (i.e., $\left.\mathrm{Si}(\mathrm{OSi})_{2}(\mathrm{OH})_{2}\right)$ the most prominent in all samples. The 
observation of differences in $\delta_{\text {iso }}$ between glasses and their corresponding crystals (which have only $\mathrm{Q}^{2}$ groups), indicated short-range structural variations between the two. Schneider et al. also showed that the distribution of the $\mathrm{Q}^{n}$ resonances for each system (and therefore the tetrahedral connectivity) was shown to correlate with the nucleation tendency of the glass, with those most similar to the corresponding crystal having the greatest nucleation rates. More recently, ${ }^{71} \mathrm{Ga}$ solid-state NMR spectroscopy has been used to investigate the variation in local $\mathrm{Ga}$ coordination environments in a series of chalcogenide and chalcohalide glasses.[280] The large quadrupolar broadening observed for ${ }^{71} \mathrm{Ga}(I=3 / 2)$ has hindered its study, particularly under MAS, but in this work the authors used wideline NMR spectroscopy (with variable offsets) of a static sample. The broad resonances observed were analysed by considering a Czjzek distribution of the EFG, and the lineshapes were shown to arise from the presence of a preferred higher-symmetry local $\mathrm{GaS}_{4}$ environment (owing to the more covalent nature of the Ga-S bonds). Changes to the local Ga environment upon addition of $\mathrm{CsCl}$ (believed to interact directly with $\mathrm{Ga}$ to form anionic complexes) were also monitored. As the $\mathrm{CsCl}$ concentration increased a transition from a covalent $\mathrm{GaS}_{4}$ environment to a more ionic $\left[\mathrm{GaS}_{4-\mathrm{x}} \mathrm{Cl}_{\mathrm{x}}\right]^{-}$environment was observed.

To obtain a more detailed structural picture of a glass it is often necessary to combined different characterisation approaches. In recent work, ${ }^{77}$ Se $(I=1 / 2)$ MAS NMR spectroscopy was supported by transition electron microscopy energy-dispersive X-ray spectroscopy to probe the structure arsenic selenide glasses.[281] ${ }^{77}$ Se MAS NMR spectra of five glasses, $\mathrm{As}_{2} \mathrm{Se}_{3}, \mathrm{AsSe}_{2}, \mathrm{AsSe}_{3}, \mathrm{AsSe}_{4.5}$ and $\mathrm{AsSe}_{6}$, were able to demonstrate the presence of different NN and NNN coordination environments, as shown in Figure 26a, where the number of position of As around Se have a noticeable effect on $\delta_{\text {iso. The upfield }}$ shift was most pronounced for Se species directly sandwiched between two As atoms. The Flory model,[281] typically applied for organic polymers, was used to investigate the distribution of chain lengths in the glasses, providing a convenient way to calculate the probability of a specific ${ }^{77}$ Se environment occurring. 
In many cases, two-dimensional spectroscopy is required to understand the broadened and overlapping lineshapes observed in NMR spectra of glasses. MQMAS spectra can often resolve signals from different chemical species, and in addition, and as described above, the shape of the MQMAS lineshape enables information on the distribution of NMR parameters (typically using a Czjzek distribution of the EFG) to be obtained. Figure $26 \mathrm{~b}$ shows ${ }^{17} \mathrm{O}$ MQMAS spectra of iron-bearing sodium silicate glasses,[282] with signals from $\mathrm{Na}-\mathrm{O}-\mathrm{Si}$ and $\mathrm{Si}-\mathrm{O}-\mathrm{Si}$ peaks clearly resolved. This study demonstrated that when replacing $\mathrm{Na}_{2} \mathrm{O}$ with $\mathrm{Fe}_{2} \mathrm{O}_{3}$ the proportion of $\mathrm{Na}-\mathrm{O}-\mathrm{Si}$ bonds decreases, suggesting that the degree of structural polymerisation increases with iron content. An alternative approach for obtaining NMR parameters from MQMAS spectra was demonstrated for borosilicate and a basaltic-like glasses by Angeli et al.,[283] who used an inversion approach. This exploits the fact that the position of the centre of gravity of a resonance in an MQMAS spectrum is dependent on both $\delta_{\text {iso }}$ and $\delta_{\mathrm{Q}}$ (and so on $\mathrm{C}_{\mathrm{Q}}$ and $\left.\eta_{Q}\right)$. Therefore, (assuming $\eta_{Q}=0.5$ for simplicity) inversion converts a $\left(\delta_{1}, \delta_{2}\right)$ spectrum to a $\left(\delta_{\text {iso }}\right.$ and $\left.C_{Q}\right)$ plot, with the dependency of the efficiency of the experiment on $C_{Q}$ and $\mathrm{rf}$ field also taken into account. From such plots the distributions in each parameters (and any correlation between them) can be read directly. The NMR parameters were also then related to structural geometry, and the distributions in the Na-O distance and the Al-O-Si bond angles for the glasses considered were determined.

The inherent structural disorder in glasses leads to complex one- and twodimensional lineshapes that can often be overlapped to some extent and are, therefore, difficult to assign to particular chemical environments. In recent years, and with the advances in computation, significant effort has focused on using a combination of DFT and MD calculations to help spectral interpretation for glasses.[89,90,276-278] As described in a recent review by Charpentier,[90] different approaches are possible. If classical MD is used, the glass is represented by an ensemble of particles, with each initial configuration generated using a random distribution of a specified number of atoms in a cubic box (consistent with the experimental composition and density). A melt-quench approach is used, carrying out the MD simulations at high temperature (i.e., simulating 
the system as a liquid), before cooling and equilibrating. Although very cost efficient, with thousands of atoms able to be considered, the need to derive specific interatomic potentials does limit transferability. Alternatively, $a b$ initio MD can be performed, although the increased cost of this approach typically limits applications to systems of $\sim 300$ atoms and over times of 100 ps. Once structures have been generated using either approach, DFT calculations (usually adopting the GIPAW algorithm), provide the distribution of NMR parameters that can be compared to experiment. As an example, Figure 26c shows ${ }^{17} \mathrm{O}$ MAS and MQMAS spectra simulated using the computed parameters from MD-generated calcium silicate sodium glasses.[89,90,284] These demonstrate that although resonances are overlapped in the conventional spectrum, they can be better resolved using MQMAS. However, overlap of the Si-O-(Na,Ca) signals with those from Si-O-Ca and $\mathrm{Si}-\mathrm{O}-\mathrm{Na}$ is expected.

Cement, used in construction to bind other materials together, is an extremely complex material, with a chemistry that is not entirely understood, despite its use in some form for many millennia. The most common form of cement, Portland cement, was patented in 1824, and is produced by blending and heating limestone, clay and calcium sulfate dihydrate at $1300-1500^{\circ} \mathrm{C}$. Given the complexity of cement systems, and their lack of any long-range order, solid-state NMR has been used extensively to investigate the structure and reactivity (typically hydration) of cement, with ${ }^{1} \mathrm{H},{ }^{27} \mathrm{Al},{ }^{29} \mathrm{Si}$ and ${ }^{43} \mathrm{Ca}$ the major nuclides exploited. NMR spectroscopy is used to determine the coordination number of the $\mathrm{Al}$ species present, and their relative proportions, while ${ }^{29} \mathrm{Si} \mathrm{NMR}$ provides information on amount of Al incorporated. In work in 2004,[285] Andersen showed using NMR spectroscopy that the presence of sodium aluminate accelerates the hydration of some components of cement, resulting in a calcium silicate hydrate phase with longer average chain lengths of $\mathrm{SiO}_{4} / \mathrm{AlO}_{4}$ tetrahedra. Detailed $\mathrm{NMR}$ studies showed that although the $\mathrm{Al} / \mathrm{Si}$ ratio of the chains increased with increasing sodium aluminate concentration, it remained fairly consistent during the hydration process, which was studied for 6 hours to 2 years. Many more solid-state NMR spectroscopy studies of the variation in local structure in cements have been performed, including one by Edwards et 
al.,[286] where ${ }^{29} \mathrm{Si}$ MAS NMR was used to determine the relative concentrations of tricalcium silicate and dicalcium silicate, which are important in the prediction of the setting properties of cement. More recently, work by Chmelka and co-workers[287,288] has demonstrated an impressive advance in the information able to be obtained from solid-state NMR spectra of anhydrous and hydrous cements. The overlapping components within high-field ${ }^{29} \mathrm{Si}$ and ${ }^{27} \mathrm{Al}$ NMR experiments were assigned to different silicate and aluminate moieties and two-dimensional correlation experiments for white Portland cement revealed the detailed molecular interactions between water and different silicate and aluminate-containing moieties during the hydration process.[287] In later work, NMR was used to follow the reaction of ${ }^{29} \mathrm{Si}$-enriched $\mathrm{Ca}_{2} \mathrm{SiO}_{5}$ with water, and was able to quantitatively monitor the local molecular composition, and the extent of silicate hydration and polymerisation, demonstrating surface passivation as the reaction slows.[288]

\subsection{Polymers}

The properties of solid polymers are determined not only by the chemical nature, structure and dynamics of the monomers from which they are composed, but also by the size and morphology of the different domains present. Many polymers display significant heterogeneity, either in their chemical make-up (e.g., co-polymers or polymer blends) or in their crystallinity, with significant proportions of both amorphous and crystalline regions typically present. The chemical and structural complexity and heterogeneity of polymers, coupled with the importance of dynamics to their structure and function makes solid-state NMR spectroscopy a vital tool for their characterization. More detailed reviews on solidstate NMR of polymers can be found in Refs. [289,290].

Even though spectral lineshapes for polymers are broadened by disorder, a range of structural information can be obtained from simple ${ }^{13} \mathrm{C}$ and ${ }^{1} \mathrm{H}$ NMR spectra (particularly at temperatures close to or above $T_{g}$, where resolution is significantly improved). It can be possible to identify (and often quantify) signals from particular sidechains, to see signals that confirm the level and type of branching in simple systems such as polyethene, or of 
the level of acetylation of monomers.[289-291] The influence of conformation on the NMR spectrum has long been studied using solution-state NMR, resulting in the well-known "gauche effect", where the shielding experienced by a C atom with $\gamma$ neighbours in a trans position, is different to that with gauche neighbours. This leads to a large number of lines that reflect the different (long-range) conformational arrangements. In the solid state resolution is poorer, but differences in short-range conformation (i.e., within a tetrameric unit) can be determined, as shown in Figure 27a[292] for the $-\mathrm{CH}_{2}$ - groups in atactic polypropylene. Information on long-range interactions that determine how the polymer chains pack is also available using NMR spectroscopy. An example is given in Figure 27b, which shows ${ }^{1} \mathrm{H} /{ }^{1} \mathrm{H}$ double-quantum correlation spectra of ureido-pyrimidone supramolecular polymers, held together by four hydrogen bonds. The tautomeric form of the end groups can change with temperature (from keto to enol), varying the hydrogen bonding arrangement, which can be followed by the changes in proximity of the types of H species shown in the spectrum.[290,293]

Many polymers are semicrystalline, and NMR spectra can provide information on the presence (and relative proportions) of the crystalline and amorphous domains that are present.[289,290] The rigid, crystalline material usually gives broader lines in the spectrum, but the higher mobility associated with the amorphous regions typically results in much narrower lineshapes. In many cases, the proportion of each component present can be determined by fitting of the lineshape, but as the structural heterogeneity increases this can become more challenging. However, the different dynamics present in the different regions also leads to different relaxation times, and it is possible to design "filters" (i.e., different series of pulses and intervals) that can select only the mobile or rigid parts of the material, ensuring that the resulting spectra contain signal only from these domains. An example of this is shown in Figure $27 \mathrm{c}$, where the ${ }^{19} \mathrm{~F}$ MAS NMR spectrum of a copolymer of vinylidene fluoride and trifluoroethylene is compared with spectra that have been filtered to show only amorphous or crystalline parts of the sample.[294] The relative sizes of polymer domains can also be probed using a variety of NMR techniques. These include "spin counting", where the number of nuclei in close 
spatial proximity can be determined by the creation of multiple-quantum coherences (i.e., measuring the number of spins that are linked by the dipolar interaction).[289,290] Information can also be obtained from spin diffusion experiments, which involve the diffusion of nuclear magnetization throughout the sample, without transport of any material. This can be measured through the variation in cross peak intensities (within a two-dimensional experiment) with increasing time, and related to the strength of the dipolar coupling (and, therefore, to distance).

Polymeric materials exhibit a variety of dynamic behavior on a range of different timescales, with rotational, vibrational and translational motion present in many systems.[289,290] The type and rate of dynamics present affects the physical properties of a polymer, how it is processed and its ultimate end use. Dynamic processes can be studied using NMR spectroscopy either by monitoring changes to anisotropic interactions (often ${ }^{13} \mathrm{C}$ CSA or ${ }^{2} \mathrm{H}$ quadrupolar lineshapes) or using two-dimensional exchange experiments. Faster motions, such as rotation of side chain acetyl or phenyl groups, result in averaging of the anisotropic interactions, and changes to the spectral lineshapes seen for static samples. Variable-temperature conventional experiments can be used for simple systems, or those where selective isotopic labeling can be used. Exchange spectroscopy is usually used to study slower motions, through the introduction of a "mixing time" (usually ms to s) into a two-dimensional experiment. When reorientational motion (either of side chain groups or from conformational changes in the polymer chain) is present experiments on static samples are typically used (often in combination with selective isotopic enrichment), enabling the difference in resonance position before and after a change in orientation to be studied. For a powder sample, this produces characteristic two-dimensional patterns that provide information on the geometry of the change. An example is shown in Figure 27d, a ${ }^{2} \mathrm{H}$ exchange NMR spectrum of polyvinylidene, where the pattern observed confirms jumps which interchange $\mathrm{C}-{ }^{2} \mathrm{H}$ bonds with orientations that differ by $113^{\circ}$, during chain rotation in the crystalline regions of the polymer.[295] For diffusional motion, e.g., chain diffusion between crystalline and amorphous regions of the polymer, exchange experiments are carried out under MAS, enabling resolution of distinct signals, and cross 
peaks in the spectrum then denote mass transport (as opposed to the spin diffusion experiments described above where only magnetization is transferred). ${ }^{13} \mathrm{C}$ MAS NMR spectroscopy has been used to directly prove chain diffusion from the crystalline to the amorphous regions in polyethene, by $180^{\circ}$ jumps in the backbone. [296,297] Arrhenius plots of jump rates against $1 / \mathrm{T}$ give the activation energy (shown to be $\sim 105 \mathrm{~kJ} \mathrm{~mol}^{-1}$ for polyethene at $360 \mathrm{~K})$.

\section{Conclusions and Perspectives}

The unique sensitivity of NMR to the local structural environment ensures it is a valuable and versatile tool for the study of disordered materials, despite the challenges involved in the acquisition of high-resolution spectra with good sensitivity. The ability to gain insight into the local atomic environment, including information on the number and type of coordinating atom(s), to measure internuclear distances and bonding interactions between atoms, and to investigate dynamics over a range of different timescales, makes NMR extremely complementary to techniques that provide information on long-range and/or average structure, or those that inform on macroscopic structure. It is clear that in order to understand in detail how and why materials behave as they do, and therefore to improve their design and development, solid-state structure must be considered on a variety of length scales simultaneously, and NMR spectroscopy provides a vital component of this approach.

Despite the benefits NMR spectroscopy provides for the study of solids, significant challenges remain and, as solid-state NMR has continued to develop over the last few decades, the long-standing aims of improving resolution and sensitivity have been joined by the need to extract detailed structural information from the complex spectral lineshapes. To date, this latter challenge has largely been approached using computation, with calculations providing a bridge between the long-range or average structural pictures provided by diffraction and the atomic-scale information provided by NMR. However, increasing reliance on computation brings its own challenges for the study of disordered 
materials, where numerous arrangements of atoms need to be considered to predict NMR spectra or to interpret complex and overlapped spectral lineshapes. This requires future development of high-throughput, efficient and accurate calculations, and advances in the theory required to include interactions such as paramagnetic shifts or spin-orbit relativity, which are not routinely included in periodic approaches.[9-11]

The improvement of NMR sensitivity continues to be a vital aim, with research efforts focussed not only on improving the efficiency of specific pulses used within individual experiments, but also on improvements in the overall sensitivity of NMR spectroscopy. In the latter case, increasing the external magnetic field results in sensitivity gains proportional to $\sim \mathrm{B}_{0}^{3 / 2}$,[1-3] although the advantage can be significantly more for quadrupolar nuclei owing to the decrease in the second-order quadrupolar broadening and, consequently, in the linewidth.[1-3,6] However, the approach generating the most interest at present is DNP where, as described above, magnetisation is transferred from unpaired electrons to nearby ${ }^{1} \mathrm{H}$ nuclei. Although enhancements close to the optimum value of 660 are yet to be realised, those of two orders that can be achieved in many cases $[44,171]$ do provide a step change in sensitivity, enabling experiments that were simply not possible before to now be performed. Whilst extremely promising, and probably the most exciting development in magnetic resonance in the last 20 years, DNP is not yet the perfect remedy to all sensitivity issues. The costs of both the hardware required, and those of day-to-day running have restricted the availability of equipment, and access is certainly not routine for the average chemist or materials scientist. Significant progress is also required to understand the polarisation transfer mechanism(s) that operate under various experimental conditions, before the development of more sophisticated experiments can advance, while the surface sensitivity of DNP (and the need for significant spin diffusion typically between ${ }^{1} \mathrm{H}$ ) ensures that additional development is required before the technique is more widely and routinely applicable to all types of materials. 
The sensitivity of NMR to the local structure should, in principle, make it ideal for the study of chemical reactivity. Although experiments have been carried out in situ and in operando, and at elevated temperatures or pressures,[152,168,298] the technical challenge of combining these with rapid MAS has restricted much of this work to the realm of specialists. Significant technological and mechanical development is required to ease the implementation of these approaches and widen their applicability. However, the possible benefits of development in this area are enormous, with NMR poised to provide information on host-guest interactions, molecular dynamics and reaction mechanism in a wide variety of systems.

Although NMR will clearly benefit from both technological and methodological developments in the future, at present it remains an important characterisation tool in the arsenal of the solid-state chemist, enabling a full and detailed structural picture on all relevant lengthscales to be obtained. For disordered solids, in particular, NMR offers structural insight that is not available from other approaches, and will play a vital role in the future design and development of new materials, and in understanding the structureproperty relationships that underpin their successful applications.

\section{Acknowledgements}

SEA would like to thank the ERC (EU FP7 Consolidator Grant 614290 "EXONMR") and the Royal Society and Wolfson Foundation for a merit award.

\section{Disclosure Statement}

The authors declare no potential conflicts of interest. 


\section{References}

[1] J. Keeler, Understanding NMR Spectroscopy, John Wiley \& Sons Ltd, Chichester, UK (2005).

[2] S.E. Ashbrook, D.M. Dawson and J.M. Griffin, in Local Structural Characterisation, Eds D.W. Bruce, D. O’Hare and R.I. Walton, John Wiley \& Sons Ltd, Chichester, UK (2014).

[3] D. Apperley, R.K. Harris and P. Hodgkinson, Solid-State NMR: Basic Principles and Practices, Momentum Press LLC, New York, $2^{\text {nd }}$ Edition (2012).

[4] K.J.D. MacKenzie and M.E. Smith, Multinuclear Solid-State NMR of Inorganic Materials, Pergamon Press, Oxford, UK (2002).

[5] E. R. Andrew, A. Bradbury and R.G. Eades, Nature 182, 1659 (1958).

[6] NMR of Quadrupolar Nuclei in Solid Materials, Eds. R.E. Wasylishen, S.E. Ashbrook and S. Wimperis, John Wiley \& Sons Ltd, Chichester, UK (2012).

[7] S.E. Ashbrook and S. Sneddon, J. Am. Chem. Soc. 136, 15440-15456 (2014).

[8] S.E. Ashbrook, Phys. Chem. Chem. Phys. 11, 6892-6905 (2009).

[9] C. Bonhomme, C. Gervais, F. Babonneau, C. Coelho, F. Pourpoint, T. Azais, S.E. Ashbrook, J.M. Griffin, J.R. Yates, F. Mauri and C.J. Pickard, Chem. Rev. 112, 5733-5779 (2012).

[10] T. Charpentier, Solid State Nucl. Magn. Reson. 40, 1-20 (2011).

[11] S.E. Ashbrook and D. McKay, Chem. Commun. 52, 7186-7204 (2016).

[12] C.J. Pickard and F. Mauri, Phys. Rev. B 63, 245101 (2001).

[13] NMR Crystallography, Eds. R.K. Harris, R.E. Wasylishen and M.J. Duer, John Wiley \& Sons Ltd, Chichester, UK (2009).

[14] C. Martineau, J. Senker and F. Taulelle, Annu. Rep. NMR Spectrosc. 82, 1-57 (2014).

[15] A. Lesage, M. Bardet and L. Emsley, J. Am. Chem. Soc. 121, 10987-10993 (1999).

[16] D.M. Grant, Chemical Shift Tensors, in Encyclopedia of Magnetic Resonance, Eds. R.K. Harris and R.E. Wasylishen, John Wiley: Chichester UK (2007).

[17] R.K. Harris, E.D. Becker, S.M.C. de Menezes, P. Granger, R.E. Hoffman and K.W. Zilm, Pure Appl. Chem. 80, 59-84 (2008). 
[18] R.E. Wasylishen, Dipolar and Indirect Coupling Tensors in Solids, in Encyclopedia of Magnetic Resonance, Eds. R.K. Harris and R.E. Wasylishen, John Wiley: Chichester UK (2007).

[19] A.J. Vega, Quadrupolar Nuclei in Solids, in Encyclopedia of Magnetic Resonance, Eds. R.K. Harris and R.E. Wasylishen, John Wiley: Chichester UK (2010).

[20] I. Bertini, C. Luchinat and S. Aime, Coord. Chem. Rev. 150, 1-292 (1996)

[21] M. Kaupp and F.H. Köhler, Coord. Chem. Rev. 253, 2376-2386 (2009).

[22] E.R. Andrew, Magic Angle Spinning, in Encyclopedia of Magnetic Resonance, Eds. R.K. Harris and R.E. Wasylishen, John Wiley: Chichester UK (2007).

[23] A. Samoson, E. Lippmaa and A. Pines, Mol. Phys. 65, 1013-1018 (1988).

[24] P. Hodgkinson, Prog. Nucl. Magn. Reson. Spectrosc. 46, 197-222 (2005).

[25] P.K. Madhu, Solid State Nucl. Magn. Reson. 35, 2-11 (2009).

[26] B.C. Gerstein, C. Clor, R.G. Pembleton and R.C. Wilson, J. Phys. Chem. 81, 565-570 (1977).

[27] S.E. Ashbrook and M.J. Duer, Concept Magn. Reson. A 28, 183-248 (2006).

[28] J. Schaefer. REDOR and TEDOR, in Encyclopedia of Magnetic Resonance, Eds. R.K. Harris and R.E. Wasylishen, John Wiley: Chichester UK (2007).

[29] L. Shao and J.J. Titman, Prog. Nucl. Magn. Reson. Spectrosc. 51, 103-137 (2007).

[30] R.M. Orr, M.J. Duer and S.E. Ashbrook, J. Magn. Reson. 174, 301-209 (2005).

[31] R.R. Ernst, Chimia, 29, 179-183 (1975).

[32] G.A. Morris and J.W. Emsley, Multidimensional NMR: an Introduction, in Encyclopedia of Magnetic Resonance, Eds. R.K. Harris and R.E. Wasylishen, John Wiley: Chichester UK (2011).

[33] S.P. Brown, M. Perez-Torralba, D. Sanz, R.M. Claramunt and L. Emsley, Chem. Commun. 1852-1853 (2002).

[34] L. Frydman and J.S. Harwood, J. Am. Chem. Soc. 117, 5367-5368 (1995).

[35] T.T. Nakashima and R.E. Wasylishen, eMagRes 2011, DOI: 10.1002/9780470034590.emrstm1200.

[36] F.A. Perras, J. Viger-Gravel, K.M.N. Burgess and D.L. Bryce, Solid State Nucl. Magn. Reson. 51-52, 1-15 (2013). 
[37] H. Colaux, D.M. Dawson and S.E. Ashbrook, J. Phys. Chem. A 118, 6018-6025 (2014).

[38] Z. Gan, J. Am. Chem. Soc. 122, 3242-3243 (2000).

[39] S.E. Ashbrook and S. Wimperis, Prog. Nucl. Magn. Reson. Spectrosc. 45, 53-108 (2004).

[40] A. Pines, J.S. Waugh and M.G. Gibby, J. Chem. Phys. 56, 1776-1777 (1972).

[41] D.P. Burum, Cross Polarization in Solids in Encyclopedia of Magnetic Resonance, Eds. R.K. Harris and R.E. Wasylishen, John Wiley: Chichester UK (2007).

[42] S. Meiboom and D. Gill, Rev. Sci. Instrum. 29, 688-691 (1958).

[43] H.Y. Carr and E.M. Purcell, Phys. Rev. 94, 630-638 (1954).

[44] Q.Z. Ni, E. Daviso, T.V. Can, E. Markhasin, S.K. Jawla, T.M. Swager, R.J. Temkin, J. Herzfeld and R.G. Griffin, Acc. Chem. Res. 46, 1933-1941 (2013).

[45] D. Lee, S. Hediger and G. de Paëpe, Solid State Nucl. Magn. Reson. 66-67, 6-20 (2015).

[46] R.W. Schurko, Acc. Chem. Res. 46, 1985-1995 (2013).

[47] S.E. Ashbrook, A.J. Berry, D.J. Frost, A. Gregorovic, C.J. Pickard, J.E. Readman and S. Wimperis, J. Am. Chem. Soc. 129, 13213-13224 (2007).

[48] K.E. Johnston, C.C. Tang, J.E. Parker, K.S. Knight, P. Lightfoot and S.E. Ashbrook, J. Am. Chem. Soc. 132, 8732-8746 (2010).

[49] K.E. Johnston, J.M. Griffin, R.I. Walton, D.M. Dawson, P. Lightfoot and S.E. Ashbrook, Phys. Chem. Chem. Phys. 13, 7565-7576 (2011).

[50] M.D. Peel, S.E. Ashbrook and P. Lightfoot, Inorg. Chem. 52, 8872-8880 (2013).

[51] S.E. Ashbrook, K.R. Whittle, G.R. Lumpkin and I. Farnan, J. Phys. Chem. B 110, 1035810364 (2006).

[52] S.E. Ashbrook, M.R. Mitchell, S. Sneddon, R.F. Moran, M. de los Reyes, G.R. Lumpkin and K.R. Whittle, Phys. Chem. Chem. Phys. 17, 9049-9059 (2015).

[53] H. Eckert, Amorphous Materials, in Encyclopedia of Magnetic Resonance, Eds. R.K. Harris and R.E. Wasylishen, John Wiley: Chichester UK (2007).

[54] R.F. Pettifer, R. Dupree, I. Farnan and U. Sternberg, J. Noncryst. Solids 106, 408-412 (1988).

[55] R. Dupree, S.C. Kohn, C.M.B. Henderson and A.M.T. Bell in Nuclear Magnetic Shieldings and Molecular Structure, Ed. J.A. Tossell, Kluwer, Dordrecht (1993).

[56] D.M. Dawson and S.E. Ashbrook, J. Phys. Chem. C 118, 23285-23296 (2014). 
[57] S. Cadars, N. Mifsud, A. Lesage, J.D. Epping, N. Hedin, B.F. Chmelka and L. Emsley, J. Phys. Chem. C 112, 9145-9154 (2008).

[58] D. Massiot, F. Fayon, M. Deschamps, S. Cadars, P. Florian, V. Montouillout, N. Pellerin, J. Hiet, A. Rakhmatullin and C. Bessada, C. R. Chimie 13, 117-129 (2010).

[59] G. Czjzek, J. Fink, F. Götz, H. Schmidt and J.M.D. Coey, Phys. Rev. B 23, 2513-2030 (1981).

[60] J.-B. d'Espinose de la Caillerie, C. Fretigny and D. Massiot, J. Magn. Reson. 192, 244251 (2008).

[61] G. Le Caer, B. Bureau and D. Massiot, J. Phys.: Condens. Matter 22, 065401 (2010).

[62] A.P.M. Kentgens, Geoderma 80, 271-306 (1997).

[63] D. Massiot, F. Fayon, M. Capron, I. King, S. Le Calve, B. Alonso, J.O. Durand, B. Bujoli, Z.H. Gan and G. Hoatson, Magn. Reson. Chem. 40, 70-76 (2002).

[64] R.L. Vold and G.L. Hoatson, J. Magn. Reson. 198, 57-72 (2009).

[65] S.E. Ashbrook, S. Antonijevic, A.J. Berry and S. Wimperis, Chem. Phys. Lett. 364, 634642 (2002).

[66] S. Antonijevic, S.E. Ashbrook, S. Biedasek, R.I. Walton, S. Wimperis and H.X. Yang, J. Am. Chem. Soc. 128, 8054-8062 (2006).

[67] W. Koch and M. C. Holthausen, A Chemist's Guide to Density Functional Theory, Wiley-VCH, Weinheim, 2nd edn, (2001).

[68] Calculation of NMR and EPR Parameters: Theory and Applications, Ed. M. Kaupp, M. Bühl, V.G. Malkin, Wiley-VCH, Weinheim (2004).

[69] S.E. Ashbrook and D.M. Dawson, Acc. Chem. Res. 46, 1964-1974 (2013).

[70] P. Hohenberg and W. Kohn, Phys. Rev. 136, B864-B871 (1964).

[71] W. Kohn and L.J. Sham, Phys. Rev. 140, A1133-A1138 (1965).

[72] R.O. Jones and O. Gunnarsson, Rev. Mod. Phys. 61, 689-746. (1989).

[73] J.P. Perdew and W. Yue, Phys. Rev. B 33, 8800-8802 (1986).

[74] J.P. Perdew, K. Burke and M. Ernzerhof, Phys. Rev. Lett. 77, 3865-3868 (1996).

[75] M.W. Lodewyk, M.R. Siebert and D.J. Tantillo, Chem. Rev. 112, 1839-1862 (2012).

[76] C.G. Van de Walle and P.E. Blöchl, Phys. Rev. B. 47, 4244-4255 (1993). 
[77] S.E. Ashbrook M. Cutajar, C.J. Pickard, R.I. Walton and S. Wimperis, Phys. Chem. Chem. Phys. 10, 5754-5764 (2008).

[78] S. Sneddon, D.M. Dawson, C.J. Pickard and S.E. Ashbrook, Phys. Chem. Chem. Phys. 16, 2660-2673 (2014).

[79] J. Klimeš and A. Michaelides, J. Chem. Phys. 137, 120901 (2012).

[80] D. Laurencin, N. Almora-Barrios, N.H. de Leeuw, C. Gervais, C. Bonhomme, F. Mauri, W. Chrzanowski, J.C. Knowles, R.J. Newport, A. Wong, Z. Gan and M.E. Smith, Biomaterials, 32, 1826-1837 (2011).

[81] V.R. Seymour, E.C.V. Eschenroeder, P.A. Wright and S.E. Ashbrook, Solid State Nucl. Magn. Reson. 65, 64-74 (2015).

[82] S.W. Reader, M.R. Mitchell, K.E. Johnston, C.J. Pickard, K.R. Whittle and S.E. Ashbrook, J. Phys. Chem. C, 113, 18874-18883 (2009).

[83] M.R. Mitchell, D. Carnevale, R. Orr, K.R. Whittle and S.E. Ashbrook, J. Phys. Chem. C, 116, 4273-4286 (2012).

[84] M.R. Mitchell, S.W. Reader, K.E. Johnston, C.J. Pickard, K.R. Whittle and S.E. Ashbrook, Phys. Chem. Chem. Phys. 13, 488-497 (2011).

[85] K.E. Johnston, M.R. Mitchell, F. Blanc, P. Lightfoot and S.E. Ashbrook, J. Phys. Chem. C 117, 2252-2265 (2013).

[86] J.M. Griffin, J.R. Yates, A.J. Berry, S. Wimperis and S.E. Ashbrook, J. Am. Chem. Soc. 132, 15651-15660 (2010).

[87] D. Carnevale, V. del Amo, D. Philp and S.E. Ashbrook, Tetrahedron, 66, 6238-6250 (2010).

[88] S. Cadars, M. Allix, D.H. Brouwer, R. Shayib, M. Suchomel, M.N. Garaga, A. Rakhmatullin, A.W. Burton, S.I. Zones, D. Massiot and B.F. Chmelka, Chem. Mater. 26, 6994-7008 (2014).

[89] T. Charpentier, S. Ispas, M. Profeta, F. Mauri and C.J. Pickard, J. Phys. Chem. B 108, 4147-4161 (2004).

[90] T. Charpentier, M.C. Menziani and A. Pedone, RSC Adv. 3, 10550-10578 (2013).

[91] C.J. Pickard and R.J. Needs, Phys. Rev. Lett. 97, 045504 (2006).

[92] C.J. Pickard and R.J. Needs, J. Phys.: Condens. Matter 23, 053201 (2011). 
[93] R.F. Moran, D. McKay C.J. Pickard, A.J. Berry, J.M. Griffin and S.E. Ashbrook, Phys. Chem. Chem. Phys. 18, 10173-10181 (2016).

[94] S.E. Ashbrook and M.E. Smith, Chem. Soc. Rev. 35, 718-735 (2006).

[95] C.P. Grey, M.E. Smith, A.K. Cheetham, C.M. Dobson and R. Dupree, J. Am. Chem. Soc. 112, 4670-4675 (1990).

[96] A.K. Cheetham, C.M. Dobson, C.P. Grey and R.J.B. Jakeman, Nature, 328, 706-707 (1987).

[97] C.P. Grey, C.M. Dobson, A.K. Cheetham and R.J.B. Jakeman, J. Am. Chem. Soc. 111, 505-511 (1989).

[98] E.J. Harvey, S.E. Ashbrook, G.R. Lumpkin and S.A.T. Redfern, J. Mater. Chem. 16, 4665-4674 (2006).

[99] M. de los Reyes, K.R. Whittle, Z. Zhang, S.E. Ashbrook, M.R. Mitchell, L-Y. Jang and G.R. Lumpkin, RSC Adv., 2013, 3, 5090-5099.

[100] F. Wolf, D. Kline and H.S. Story, J. Chem. Phys. 53, 3538-3543 (1970).

[101] S.E. Ashbrook, L. Le Polles, R. Gautier, C.J. Pickard and R.I. Walton, Phys. Chem. Chem. Phys. 8, 3423-3431 (2006).

[102] D.R. Modeshia, R.J. Darton, S.E. Ashbrook and R.I. Walton, Chem. Commun. 68-70 (2009).

[103] D.R. Modeshia, R.I. Walton, M.R. Mitchell and S.E. Ashbrook, Dalton Trans. 39, 60316036 (2010).

[104] M.H. Harunsani, D.I. Woodward, M.D. Peel, S.E. Ashbrook and R.I. Walton, J. SolidState Chem. 207, 117-125 (2013).

[105] L. Buannic, F. Blanc, D.S. Middlemiss and C.P. Grey, J. Am. Chem. Soc. 134, 1448314498 (2012).

[106] H.Y. Playford, A.C. Hannon, M.G. Tucker, D.M. Dawson, S.E. Ashbrook, R.J. Kastiban, J. Sloan and R.I. Walton, J. Phys. Chem. C. 118, 16188-16198 (2014).

[107] F. Blanc, L. Spencer and G.R. Goward, Quadrupolar NMR of Ionic Conductors, Batteries, and Other Energy-related Materials, eMagRes (2011).

[108] L. Shen and L. Peng, Chin. J. Catal. 36, 1494-1504 (2015). 
[109] L. O'Dell and C.I. Ratcliffe, Quadrupolar NMR to Investigate Dynamics in Solid Materials, eMagRes (2011).

[110] M.R. Hampson, J.S.O. Evans and P. Hodgkinson, J. Am. Chem. Soc. 127, 15175-15181 (2005).

[111] P. Hodgkinson and M.R. Hampson, Solid State Nucl. Magn. Reson. 30, 98-105 (2006).

[112] A. Soleilhavoup, M.R. Hampson, S.J. Clark, J.S.O. Evans and P. Hodgkinson, Magn. Reson. Chem. 45, 5144-5155 (2007).

[113] M.R. Hampson, P. Hodgkinson, J.S.O. Evans, R.K. Harris, I.J. King, S. Alena and F. Fayon, Chem. Commun. 392-393 (2004).

[114] J. Emery, O. Bohnke, P. Florian and K. Marzouk, J. Phys. Chem. B. 109, 20680-20689 (2005).

[115] G. Kim, J.M. Griffin, F. Blanc, S.M. Haile and C.P. Grey, J. Am. Chem. Soc. 137, 38673876 (2015).

[116] W.A. Deer, R.A. Howie and J. Zussman, An Introduction to the Rock-Forming Minerals, Pearson Education (1992).

[117] A.E. Ringwood, Composition and Petrology of the Earth's Mantle, McGraw-Hill, New York, (1975).

[118] J.F. Stebbins and X. Xue, Rev. Mineral. Geochem. 78, 605-653 (2014)

[119] R J. Kirkpatrick, Geological Applications, eMagRes Online, Wiley, (2007).

[120] J.F. Stebbins, Quadrupolar NMR in Earth Sciences, eMagRes Online, Wiley, (2011).

[121] J.M. Griffin and S.E. Ashbrook, Annu. Rep. NMR Spectrosc. 79, 241-332 (2013).

[122] S.E. Ashbrook and D.M. Dawson, "NMR Spectroscopy of Minerals and Allied Materials", in Nuclear Magnetic Resonance: Volume 45 Ed. V. Ramesh, RSC, Cambridge, UK (2016).

[123] G. Engelhardt and D. Michel High-Resolution Solid-State NMR of Silicates and Zeolites. Wiley, New York (1987)

[124] P.F. Barron, P. Slade and R.L. Frost, J. Phys. Chem. 89, 3880-3885 (1985).

[125] P. Florian, E. Veron, T.F.G. Green, J.R. Yates and D. Massiot, Chem. Mater. 24, 40684079 (2012).

[126] A. Bosenick, C.A. Geiger and B.L. Phillips, Am Mineral 84, 1422- 1432 (1999). 
[127] A.C. Palke, J.F. Stebbins, C.A. Geiger and G. Tippelt, Am. Mineral. 100, 536-547 (2015).

[128] R. Langner, M. Fechtelkord, A. Garcia, E.J. Palin and J. Lopez-Solano, Am. Mineral. 97, 341-352 (2012).

[129] M. Fechtelkord and R. Langner, Am. Mineral. 98, 120-131 (2013).

[130] S. Cadars, R. Geugan, M.N. Garga, X. Bourrat, L. Le Forestier, F. Fayon, T.V. Huynh, T. Allier, Z. Nour and D. Massiot, Chem. Mater. 24, 4376-4389 (2012).

[131] S. Cadars, G. Layrac, C Gérardin, M. Deschamps, J.R. Yates, D. Tichit and D. Massiot, Chem. Mater. 23, 2821-2831 (2011).

[132] D. Hirsemann, T.K.J. Köster, J. Wack, L. van Wüllen, J. Breu and J. Senker, Chem. Mater. 23, 3152-3158 (2011).

[133] J. Rocha, J. Phys. Chem. B 103, 9801-9804 (1999).

[134] I. Farnan, H. Cho and W.J. Weber, Nature 445, 190-193 (2007).

[135] I. Farnan, H. Cho, W.J. Weber, R.D. Scheele, N.R. Johnson and A.E. Kozelisky, Rev. Sci. Instrum. 75, 5232-5236 (2004).

[136] I. Farnan and E.K.H. Salje, J. Appl. Phys. 89, 2084-2090 (2001).

[137] http: / / ccmmp.ph.qmul.ac.uk/ kostya/ rad.html, accessed August 2016

[138] I. Farnan, E. Balan, C.J. Pickard and F. Mauri, Am. Mineral. 88, 1663-1667 (2003).

[139] E. Balan, F. Mauri, C.J. Pickard, I. Farnan and G. Calas, Am. Mineral. 88, 1769-1777 (2003).

[140] S.E. Ashbrook and I. Farnan, Solid State Nucl. Magn. Reson. 26, 105-112 (2004).

[141] A.C. Palke, J.F. Stebbins, D.J. Frost and C.A. McCammon, 97, 1955-1964 (2012).

[142] S.C. Kohn. Am. Mineral. 81, 1523-1526 (1996).

[143] H. Keppler and M. Rauch, Phys. Chem. Mineral. 27, 371-376 (2000).

[144] H. Eckert, J.P. Yesinowski, L.A. Silver and E.M. Stolper, J. Phys. Chem. 92, 2055-2064 (1988).

[145] S.E. Ashbrook, A.J. Berry and S. Wimperis, J. Am. Chem. Soc. 123, 6360-6366 (2001).

[146] J.M. Griffin, S. Wimperis, A.J. Berry, C.J. Pickard and S.E. Ashbrook, J. Phys. Chem. C 113, 465-471 (2009). 
[147] J.M. Griffin, A.J. Miller, A.J. Berry, S. Wimperis and S.E. Ashbrook, Phys. Chem. Chem. Phys. 12, 2989-2998 (2010).

[148] J.R. Smyth, Am. Mineral. 79, 1021-1024 (1994).

[149] S.C. Kohn, R.A. Brooker, D.J. Frost, A.E. Slesinger and B.J. Wood, Am. Mineral. 87, 293-301 (2002).

[150] J.M. Griffin, A.J. Berry, D.J. Frost, S. Wimperis and S.E. Ashbrook, Chem. Sci. 4, 15231538 (2013).

[151] P.A. Wright, Microporous Framework Solids, 1st edn., The Royal Society of Chemistry, Cambridge, UK, (2008).

[152] S.E. Ashbrook, D.M. Dawson and V.R. Seymour, Phys. Chem. Chem. Phys. 16, 82238242 (2014).

[153] C. Bonhomme, C. Gervais and D. Laurencin, Prog. Nucl. Magn. Reson. Spectrosc. 77, 1-48 (2014).

[154] L. Mafra and J. Klinowski, Molecular Sieves: Crystalline Systems, eMagRes, Wiley (2013). DOI: 10.1002/9780470034590.emrstm1325.

[155] L. Mafra and J. Klinowski, Advanced Solid State NMR Techniques for the Study of Molecular Sieves, eMagRes, Wiley (2013). DOI: 10.1002/9780470034590.emrstm1307.

[156] E. Brunner, J. Mol. Struct. 355, 61-85 (1995).

[157] M. Werner, N. Rothermel, H. Breitzke, T. Gutmann and G. Buntkowsky, Isr. J. Chem. 54, 60-73 (2014).

[158] M. Hunger, Catal. Rev. Sci. Eng. 39, 345-393 (1997).

[159] W. Zhang, S. Xu, X. Han and X. Bao, Chem. Soc. Rev. 41, 192-210 (2012).

[160] H.C. Hoffman, M. Debowski, P. Müller, S. Paasch, I. Senkovska, S. Kaskel and E. Brunner, Materials 5, 2537-2572 (2012).

[161] M. Hartmann and L. Kevan, Res. Chem. Intermed. 28, 625-695 (2002).

[162] A. Zheng, S.-J. Huang, Q. Wang, H. Zhang, F. Deng and S.-B. Liu, Chin. J. Catal. 34, 436-491 (2013).

[163] G. Engelhardt, D. Zeigan, E. Lippmaa and M. Mägi, Z. Anorg. Allg. Chem. 468, 35-38 (1980). 
[164] J. Klinowski, J.M. Thomas, C.A. Fyfe and J.S. Hartman, J. Phys. Chem. 85, 2590-2594 (1981).

[165] J.F. Stebbins, P. Zhao, S.K. Lee and X. Cheng, Am. Mineral. 84, 1680-1684 (1999).

[166] M.T. Melchior, D.E.W. Vaughan and C.F. Pictroski, J. Phys. Chem. 99, 6128-6144 (1995).

[167] D.I. Kolokolov, S.S. Arzumanov, D. Freude, J. Haase and A.G. Stepanov, J. Phys. Chem. C 120, 4993-5000 (2016).

[168] I.I. Ivanova and Y.G. Kolyagin, Chem. Soc. Rev. 39, 5018-5050 (2010).

[169] W.J. Roth, P. Nachtigall, R.E. Morris, P.S. Wheatley, V.R. Seymour, S.E. Ashbrook, P. Chlubna, L. Grajciar, M. Položij, A. Zukal, O. Shvets and J. Čejka, Nature Chem. 5, 628-633 (2013).

[170] P.S. Wheatley, P. Chlubná-Eliášová, H. Greer, W. Zhou, V.R. Seymour, D.M. Dawson, S.E. Ashbrook, A.B. Pinar, L.B. McCusker, M. Opanasenko, J. Čejka, and R.E. Morris, Angew. Chem. Int. Ed. 53, 13210-13214 (2014).

[171] A.J. Rossini, A. Zagdoun M. Lelli, A. Lesage, C. Copéret and L. Emsley, Acc. Chem. Res. 46, 1942-1951 (2013).

[172] A. Zagdoun, G. Casano, O. Ouari, G. Lapadula, A.J. Rossini, M. Lelli, M. Baffert, D. Gajan, L. Veyre, W.E. Maas, M. Rosay, R.T. Weber, C. Thieuleux, C. Coperet, A. Lesage, P. Tordo and L. Emsley, J. Am. Chem. Soc. 134, 2284-2291 (2012).

[173] M. Castro, V.R. Seymour, D. Carnevale, J.M. Griffin, S.E. Ashbrook, P.A. Wright, D.C. Apperley, J.E. Parker, S.P. Thompson, A. Fecant and N. Bats, J. Phys. Chem. C 114, 12698-12710 (2010).

[174] V.R. Seymour, E.C.V. Eschenroeder, M. Castro, P.A. Wright and S.E. Ashbrook, CrystEngComm 15, 8668-8679 (2013).

[175] F. Taulelle, M. Pruski, J.-P. Amoureux, D. Lang, A. Bailly, C. Huguenard, M. Haouas, C. Gérardin, T. Loiseau and G. Férey, J. Am. Chem. Soc. 121, 12148-12153 (1999).

[176] C. Martineau, M. Mellot-Draznieks and F. Taulelle, Phys. Chem. Chem. Phys. 13, 18078-18087 (2011).

[177] C. Martineau, F. Engelke and F. Taulelle, J. Magn. Reson. 212, 311-319 (2011). 
[178] C. Martineau, B. Bouchevreau, Z. Tian, S.-J. Lohmeier, P. Behrens and F. Taulelle, Chem. Mater. 23, 4799-4809 (2011).

[179] Z. Han, A.L. Picone, A.M.Z. Slawin, V.R. Seymour, S.E. Ashbrook, W. Zhou, S.P. Thompson, J.E. Parker and P.A. Wright, Chem. Mater. 22, 338-346 (2010).

[180] L. Vidal, V. Grammlich, J. Patarin and Z. Gabelica, Eur. J. Solid State Inorg. Chem. 35, 545-563 (1998).

[181] K.O. Kongshaug, H. Fjellvåg, B. Klewe and K.P. Lillerud, Micropor. Mesopor. Mater. 39, 333-339 (2000).

[182] J.M. Griffin, L. Clark, V.R. Seymour, D.W. Aldous, D.M. Dawson, D. Iuga, R.E. Morris and S.E. Ashbrook, Chem. Sci. 3, 2293-2300 (2012).

[183] D. Goldfarb, H.-X. Li and M.E. Davis, J. Am. Chem. Soc. 114, 3690-3697 (1992).

[184] M.J. Duer, H. He, W. Kolodziejski and J. Klinowski, J. Phys. Chem. 98, 1198-1204 (1994).

[185] S. Ishimaru, K. Gotoh, M. Ichikawa and R. Ikeda, Micropor. Mesopor. Mater. 51, 1722 (2002).

[186] Y. Komori and S. Hayashi, J. Phys. Chem. B 110, $90-96$ (2006).

[187] A. Tuel, S. Caldarelli, A. Meden, L. B. McCusker, C. Baerlocher, A. Ristic, N. Rajic, G. Mali and V. Kaucic, J. Phys. Chem. B 104, 5697-5705 (2000).

[188] G. Poulet, P. Sautet and A. Tuel, J. Phys. Chem. B, 106, 8599-8608 (2002).

[189] S.E. Ashbrook, M. Cutajar, J.M. Griffin, Z.A.D. Lethbridge, R. I. Walton and S. Wimperis, J. Phys. Chem. C 113, 10780-10789 (2009).

[190] H.O. Pastore, S. Coluccia and L. Marchese, Annu. Rev. Mater. Res. 35, 351-395 (2005).

[191] R. Vomscheid, M. Briend, M.J. Peltre, P.P. Man and D. Barthomeuf, J. Phys. Chem. 98, 9614-9618 (1994).

[192] H.O. Pastore, É.C. de Oliviera, G. B. Superti, G. Gatti and L. Marchese, J. Phys. Chem. C 111, 3116-3129 (2007).

[193] G.A.V. Martins, G. Berlier, S. Coluccia, H.O. Pastore, G.B. Superti, G. Gatti and L. Marchese, J. Phys. Chem. C 111, 330-339 (2007).

[194] A. Philippou, F. Salehirad, D.-P. Luigi and M.W. Anderson, J. Phys. Chem. B. 102, 8974-8977 (1998). 
[195] L. Canesson and A. Tuel, Chem. Commun. 241-242 (1997).

[196] G. Mali, A. Ristić and V. Kaućić, J. Phys. Chem. B, 109, 10711-10716 (2005).

[197] R.L. Bedard, C.L. Bowes, N. Coombs, A.J. Holmes, T. Jiang, S.J. Kirkby, P.M. Macdonald, A.M. Malek, G.A. Ozin, S. Petrov, N. Plavac, R.A. Ramik, M.R. Steele, and D. Young, J. Am. Chem. Soc. 115, 2300-2313 (1993).

[198] M. Amri, S.E. Ashbrook, D.M. Dawson, J.M. Griffin, R.I. Walton and S. Wimperis, J. Phys. Chem. C 116, 15048-15057 (2012).

[199] X. Kong, H. Deng, F. Yan, J. Kim, J.A. Swisher, B. Smit, O.M. Yaghi and J. A. Reimer, Science 341, 882-885 (2013).

[200] K.C. Jayachandrababu, R.J. Verploegh, J. Leisen, R.C. Nieuwendaal, D.S. Sholl and S. Nair J. Am. Chem. Soc. 138, 7325-7336 (2016).

[201] P. He, B.E.G. Lucier, V.V. Terskikh, Q. Shi, J. Dong, Y. Chu, A. Zheng, A. Sutrisno and Y. Huang, J. Phys. Chem. C 118, 23728-23744 (2014).

[202] L. Mitchell, P. Williamson, B. Ehrlichová, A.E. Anderson, V.R. Seymour, S.E. Ashbrook, N. Acerbi, L.M. Daniels, R.I. Walton, M.L. Clarke and P.A. Wright, Chem. Eur. J. 20, 17185-17197 (2014).

[203] F. Gul-E-Noor, B. Jee, M. Mendt, D. Himsl, A. Pöppl, M. Hartmann, J. Haase, H. Krautscheid and M. Bertmer, J. Phys. Chem. C 116, 20866-20873 (2012).

[204] A. Cadiau, S. Auguste, F. Taulelle, C. Martineau and K. Adil, CrystEngComm 15, 3430-3435 (2013).

[205] P. He, J. Xu, V.V. Terskikh, A. Sutrisno, H.-Y. Nie and Y. Huang, J. Phys. Chem. C 117, 16953-16960 (2013).

[206] Y. Han, J.-R. Li, Y. Xie and G. Guo, Chem. Soc. Rev. 43, 5952-5981 (2014).

[207] T. Wittmann, R. Siegel, N. Reimer, W. Milius, N. Stock and J. Senker, Chem. Eur. J. 21, 314-323 (2015).

[208] J.P.S. Mowat, S.R. Miller, J.M. Griffin, V.R. Seymour, S.E. Ashbrook, S.P. Thompson, D. Fairen-Jimenez, A.-M. Banu, T. Düren and P.A. Wright, Inorg. Chem. 50, 10844-10858 (2011).

[209] D.I. Kolokolov, H. Jobic, A.G. Stepanov, V. Guillerm, T. Devic, C. Serre and G. Férey, Angew. Chem. Int. Ed. 49, 4791-4794 (2010). 
[210] J.P.S. Mowat, V.R. Seymour, J.M. Griffin, S.P. Thompson, A.M.Z Slawin, D. FairenJimenez, T. Düren, S E. Ashbrook and P.A. Wright, Dalton Trans., 41, 3937-3941 (2012).

[211] T. Loiseau, C. Serre, C. Huguenard, G. Fink, F. Taulelle, M. Henry, T. Bataille and G. Férey, Chem. Eur. J., 10, 1373-1382 (2004).

[212] M.I.H. Mohideen, B. Xiao, P.S. Wheatley, A.C. McKinlay, Y. Li, A.M.Z. Slawin, D.W. Aldous, N.F. Cessford, T. Düren, X. Zhao, R. Gill, K.M. Thomas, J.M. Griffin, S.E. Ashbrook and R.E. Morris, Nature Chem. 3, 304-310 (2011).

[213] D.M. Dawson, L.E. Jamieson, M.I.H. Mohideen, A.C. McKinlay, I.A. Smellie, R. Cadou, N.S. Keddie, R.E. Morris and S.E. Ashbrook, Phys. Chem. Chem. Phys. 15, 919-929 (2013).

[214] A.C. McKinlay, P.K. Allan, C.L. Renouf, M.J. Duncan, P.S. Wheatley, S.J. Warrender, D.M. Dawson, S.E. Ashbrook, B. Gil, B. Marszalek, T. Düren, J. Williams, C. Charrier, D. Mercer, S.J. Teat and R.E. Morris, Appl. Phys. Lett. Mater. 2, 124108 (2014).

[215] X. Kong, E. Scott, W. Ding, J.A. Mason, J.R. Long and J.A. Reimer, J. Am. Chem. Soc. 134, 14341-14344 (2012).

[216] W.D. Wang, B.E.G. Lucier, V.V. Terskikh, W. Wang and Y. Huang, J. Phys. Chem. Lett. 5, 3360-3365 (2014).

[217] F. Gul-E-Noor, D. Michel, H. Krautscheid, J. Haase and M. Bertmer, J. Chem. Phys. 139, 034202 (2013).

[218] L.-J. Li, P.-Q. Liao, C.-T. He, Y.-S. Wei, H.-L. Zhou, J.-M. Lin, X.-Y. Li and J.-P. Zhang, J. Mater. Chem. A 3, 21849-21855 (2015).

[219] J.J. Gassensmith, H. Furukawa, R.A. Smaldone, R.S. Forgan, Y.Y. Botros, O.M. Yaghi and J.F. Stoddart, J. Am. Chem. Soc. 133, 15312-15315 (2011).

[220] F. Blanc, S.Y. Chong, T.O. McDonald, D.J. Adams, S. Pawsey, M. A. Caporini and A.I. Cooper, J. Am. Chem. Soc. 135, 15290-15293 (2013).

[221] R.K. Harris, J. Pharm. Pharmacol. 59, 225-239 (2007).

[222] S.P. Brown, Macromol Rapid Commun. 30, 688-716 (2009).

[223] R.K. Harris, Analyst 131, 351 (2006).

[224] M. Potrzebowski, Crystallography and NMR: Applications to Organic and Pharmaceutical Chemistry, eMagRes (2008). 
[225] S.P. Brown and H.W. Spiess, Chem. Rev. 101, 4125-4155 (2001).

[226] S.P. Brown, Hydrogen Bonding in Crystalline Organic Solids, eMagRes (2008).

[227] A.Othman, J.S.O. Evans, I.R. Evans, R.K. Harris and P. Hodgkinson, J. Pharm. Sci. 96, 1380-1397 (2007).

[228] K.D.M. Harris, Appl. Petrochem. Res. (2016) DOI 10.1007/s13203-016-0152-3

[229] C.E. Hughes, P.A. Williams, T.R. Peskett and K.D.M. Harris, J. Phys. Chem. Lett. 3, 3176-3181 (2012).

[230] K.D.M. Harris, C.E. Hughes and P.A. Williams, Solid State Nucl. Magn. Reson. 65, 107-113 (2015).

[231] C.E. Hughes and K.D.M. Harris, J. Phys. Chem. A 112, 6808-6810 (2008).

[232] C.E. Hughes and K.D.M. Harris, Chem. Commun. 46, 4982-4984 (2010).

[233] T.N. Pham, S. Masiero, G. Gottarelli and S.P. Brown, J. Am. Chem. Soc. 127, 1601816019 (2005).

[234] P. Sanz Camacho, K.S. Athukorala Arachchige, A.M.Z. Slawin, T.F.G. Green, J.R. Yates, D.M Dawson, J.D. Woollins and S.E. Ashbrook, J. Am. Chem. Soc. 137, 6172-6175 (2015).

[235] P. Sanz Camacho, D. McKay, D.M. Dawson, C. Kirst, J.R. Yates, T.F.G. Green, D.B. Cordes, A.M.Z. Slawin, J.D. Woollins and S.E. Ashbrook, Inorg. Chem. (2016) DOI: 10.1021/acs.inorgchem.6b01121

[236] M. Kibalchenko, D. Lee, L. Shao, M.C. Payne, J.J. Titman and J.R. Yates, Chem. Phys. Lett. 498, 270-276 (2010).

[237] G.M. Day, W.D.S. Motherwell and W. Jones, Phys. Chem. Chem. Phys. 9, 1693-1704 (2007).

[238] G.M. Day, Crystallogr. Rev. 17, 3-52 (2011).

[239] E. Salager, G.M. Day, R.S. Stein, C.J. Pickard, B. Elena and L. Emsley, J. Am. Chem. Soc., 132, 2564-2566 (2010).

[240] R.K. Harris, P. Hodgkinson, T. Larsson and A. Muruganatham, J. Pharm. Biomed. Anal. 38, 858-864 (2005).

[241] S. Sanchez, F. Ziarelli, S. Viel, C. Delaurent and S. Caldarelli, J. Pharm. Biomed. Anal. 47, 683-387 (2008). 
[242] J. Brus, M. Urbanova, I. Sedenkova and H. Brusoav, Int. J. Pharm. 409, 62-74 (2011).

[243] D.C. Apperley, A.H. Forster, R. Fournier, R.K. Harris, P. Hodgkinson, R.W. Lancaster and T. Rades, Magn. Reson. Chem. 43, 881-892 (2005)

[244] S. Cadars, A. Lesage, C.J. Pickard, P. Sautet and L. Emsley, J. Phys. Chem. 113, 902911 (2009).

[245] D. Sakellariou, S.P. Brown, A. Lesage, S. Hediger, M. Bardet, C.A. Meriles, A. Pines and L. Emsley, J. Am. Chem. Soc. 125, 4376-4380 (2003).

[246] A.D. Bond, C. Cornett, F.H. Larsen, H. Qu, D. Raijada and J. Rantanen, Cryst. Growth Des. 13, 3665-3671 (2013).

[247] H.E. Kerr, L.K. Softley, K. Suresh, A. Nangia, P. Hodgkinson and I.R. Evans CrystEngComm 17, 6707-6715 (2015).

[248] D.C. Apperley, A.F. Markwell, I. Frantsuzov, R.K. Harris and P Hodgkinson, Phys. Chem. Chem. Phys. 15, 6422-6430 (2013).

[249] X. Kong, L.A. O'Dell, V. Terskikh, E. Ye, R. Wang and G. Wu, J. Am. Chem. Soc. 134, 14609-14617 (2012).

[250] M. Cutajar, S.E. Ashbrook and S. Wimperis, Chem. Phys. Lett. 423, 276-281 (2006).

[251] A.J. Ilott, S. Palucha, A.S. Batsanov, M.R. Wilson and P. Hodgkinson, J. Am. Chem. Soc. 1132, 5179-5185 (2010).

[252] J.C. Lindon, O.P. Beckonert, E. Holmes and J.K. Nicholson, Prog. Nucl. Magn. Reson. Spectrosc. 55, 79-100 (2009).

[253] D.A. Middleton, Crystallography and NMR: Applications to Structural Biology, eMagRes (2009) DOI: 10.1002/9780470034590.emrstm1109.

[254] M.J. Duer, J. Magn. Reson. 253, 98-110 (2015).

[255] N. Nassif, F. Martineau, O. Syzgantseva, F. Gobeaux, M. Willinger, T. Coradin, S. Cassaignon, T. Azaïs and M.M. Giraud-Guille, Chem. Mater. 22, 3653-3663 (2010). [256] C.H. Yoder, J.D. Pasteris, K.N. Worcester and D.V. Schermerhorn, Calcif. Tissue Int. 90, 60-67 (2012).

[257] H. Yi, E. Balan, C. Gervais, L. Ségalen, D. Roche, A. Person, F. Fayon, G. Morin and F. Babonneau, Acta Biomin. 10, 3952-3958 (2014). 
[258] N.V. Bulina, M.V. Chaikina, A.S. Andreev, O.B. Lapina, A.V. Ishchenko, I.Y. Prosanov, K.B. Gerasimov and L.A. Solovyov, Eur. J. Inorg. Chem. 2014, 4810-4825 (2014). [259] E. Davies, M.J. Duer, S.E. Ashbrook and J.M. Griffin, J. Am. Chem. Soc. 134, 1250812515 (2012).

[260] E. Davies, K.H. Müller, W.C. Wong, C.J. Pickard, D.G. Reid, J.N. Skepper and M.J. Duer, Proc. Natl. Acad. Sci. USA 111, E1354-E1363 (2014).

[261] J. Xu, P. Zhu, Z. Gan, N. Sahar, M. Tecklenburg, M.D. Morris, D.H. Kohn and A. Ramamoorthy, J. Am. Chem. Soc. 132, 11504-11509 (2010).

[262] D. Laurencin, A. Wong, W. Chrzanowski, J.C. Knowles, D. Qiu, D. M. Pickup, R.J. Newport, Z. Gan, M.J. Duer and M.E. Smith, Phys. Chem. Chem. Phys. 12, 1081-1091 (2010).

[263] C. Groeger, K. Lutz and E. Brunner, Prog. Nucl. Magn. Reson. Spectrosc. 54, 54-68 (2009).

[264] B. Tesson, S. Masse, G. Laurent, J. Maquet, J. Livage, V. Martin-Jezequel and T. Coradin, Anal. Bioanal. Chem. 390, 1889-1898 (2008).

[265] A. Akiva-Tal, S. Kababya, Y.S. Balazs, L. Glazer, A. Berman, A. Sagi and A. Schmidt, Proc. Natl. Acad. Sci. USA 108, 14763-14768 (2011).

[266] R.J. Reeder, Y. Tang, M.P. Schmidt, L.M. Kubista, D.F. Cowan and B.L. Phillips, Cryst. Growth Des. 13, 1905-1914 (2013).

[267] V. Righi, C. Constantinou, M. Kesarwani, L.G. Rahme and A.A. Tzika, Biomed. Rep. 1, 707-712 (2013).

[268] B.J. Blaise, J. Giacomotto, B. Elena, M.E. Dumas, P. Toulhoat, L. Ségalat and L. Emsley, Proc. Natl. Acad. Sci. USA 104, 19808-19812 (2007).

[269] A. Wong, X. Li, L. Molin, F. Solari, B. Elena-Hermann and D. Sakellariou, Anal. Chem. 86, 6064-6070 (2014).

[270] V. Righi, Y. Apidiankis, D. Mintzopoulous, L. Astrakas, L.G. Rahme and A.A. Tzkia, Int. J. Mol. Med. 26, 175-184 (2010).

[271] V. Righi, Y. Apidiankis, N. Psychogios, L. Astrakas, L.G. Rahme, R.G. Tompkins and A.A. Tzkia, Int J. Mol. Med. 34, 327-333 (2014). 
[272] V. Sarou-Kanian, N. Joudiou, F. Louat, M. Yon, F. Szeremeta, S. Même, D. Massiot, M. Decoville, F. Fayon and J.-C. Beloeil, Sci. Rep. 5, 9872 (2015).

[273] M. Edén, Annu. Rep. Prog. Chem. C 108, 177-221 (2012).

[274] M. Edén, Ann. Rep. NMR Spectrosc. 86, 237-331 (2015).

[275] H. Eckert, Prog. Nucl. Magn. Reson. Spectrosc. 24, 159-293 (1992).

[276] M. Benoit, M. Profeta, F. Mauri, C.J. Pickard and M.E. Tuckerman, J. Phys. Chem. B 2005, 109, 6052-6060 (2005).

[277] A. Pedone, E. Gambuzzi, G. Malavasi and M.C. Menziani, Theor. Chem. Acc. 131, 1147-1157 (2012).

[278] A. Pedone, T. Charpentier and M.C. Menziani, Phys. Chem. Chem. Phys. 12, 60546066 (2010).

[279] J. Schneider, V.R. Mastelaro, H. Panepucci and E.D. Zanotto, J. Non. Cryst. Solids 273, 8-18 (2000).

[280] Y. Ledemi, B. Bureau, G. Le Caër, L. Calvez, C. Roiland, G. Tricot, P. Florian, V. Nazabal and D. Massiot, J. Non. Cryst. Solids 383, 216-221 (2014).

[281] M. Deschamps, C. Genevois, S. Cui, C. Roiland, L. Le Polles, E. Furet, D. Massiot and B. Bureau, J. Phys. Chem. C 119, 11852-11857 (2015).

[282] H.I. Kim, J.C. Sur and S.K. Lee, Geochim. Cosmochim. Acta 173, 160-180 (2016).

[283] F. Angeli, T. Charpentier, P. Faucon and J.-C. Petit, J. Phys. Chem. B 103, 10356-10364 (1999).

[284] A. Pedone, E. Gambuzzi and M.C. Menziani, J. Phys. Chem. C 116, 14599-14609 (2012).

[285] M. D. Andersen, H. J. Jakobsen, and J. Skibsted, Cem. Concr. Res. 34, 857-868 (2004).

[286] C.L. Edwards, L.B. Alemany and A.R. Barron, Ind. Eng. Chem. Res. 46, 5122-5130 (2007).

[287] A. Rawal, B.J. Smith, G.L. Athens, C.L. Edwards, L. Roberts, V. Gupta and B.F. Chmelka, J. Am. Chem. Soc. 132, 7321-7337 (2010).

[288] E. Pustovgar, R.P. Sangodkar, A.S. Andreev, M. Palacios, B.F. Chmelka, R.J. Flatt and J.-B. d'Espinose de Lacaillerie, Nature Comm. 7, 10952 (2016). 
[289] H. W. Spiess, Polymer Dynamics and Order from Multidimensional Solid-State NMR, eMagRes (2007).

[290] K. Saalwachter and H. Spiess, Solid-State NMR of Polymers, in Polymer Science: A Comprehensive Reference, Eds. K. Matyjaszewski and M. Möller (2012).

[291] K. Klimke, M. Parkinson, C. Piel, W. Kaminsky, H.W. Spiess and M. Wilhelm, Macromol. Chem. Phys. 207, 382-395 (2006).

[292] K. Zemke, K. Schmidt-Rohr and H. W. Spiess, Acta Polym. 45, 148-159 (1994).

[293] I. Schnell, B. Langer, S.H.M. Sontjens, R.P. Sijbesma, M.H.P. van Genderen and H.W. Spiess, Phys. Chem. Chem. Phys. 4, 3750-3758 (2002).

[294] P. Hazendonk, R.K. Harris, S. Ando and P. Avalle, J. Magn. Reson. 162, 206-216 (2003).

[295] J. Hirschinger, D. Schaefer, H W. Spiess and A.J. Lovinger, Macromolecules, 24, 24282433 (1991).

[296] K. Schmidt-Rohr and H.W. Spiess, Macromolecules 24, 5288-5293 (1991).

[297] M. Mansfield and R.H. Boyd, J. Polym. Sci., Polym. Phys. Ed. 16, 1227-1252 (1978).

[298] M. Hunger, Prog. Nucl. Magn. Reson. Spectrosc. 53, 105-127 (2008). 


\section{Figure captions}

Figure 1. (a) Ordered crystalline $A B$ material, (b) $A\left(B_{0.5} C_{0.5}\right)$, with random distribution of $B$ and $\mathrm{C}$ atoms in the crystal lattice, $(\mathrm{c}) \mathrm{A}_{0.75} \mathrm{~B}$, with a random distribution of $\mathrm{A}$-site vacancies and (d) amorphous AB.

Figure 2. (a) The application of an external magnetic field, $\mathrm{B}_{0}$, aligns the nuclear spins and lifts the degeneracy of the spin $I=1 / 2$ nuclear spin energy levels through the Zeeman interaction. (b) Vector model representation of the bulk magnetisation vector, $\mathbf{M}$, aligned along the z-axis of the rotating frame. The application of a pulse along the $\mathrm{x}$-axis causes nutation of $\mathbf{M}$ in the yz-plane, through a flip angle $\beta$. $\mathbf{M}$ then undergoes free precession in the xy-plane at a frequency $\Omega$. (c) Fourier transformation (FT) of the resulting time-domain signal, $S(t)$, yields the frequency-domain spectrum, $S(\omega)$.

Figure 3. (a) Schematic powder pattern lineshapes for a spin $I=1 / 2$ nucleus subject to a CSA with $\eta_{\mathrm{CS}}=0$ and 1. (b) Pake doublet lineshape resulting from a dipolar interaction between $\mathrm{I}=\mathrm{S}=1 / 2$ nuclei. (c) Gaussian-like lineshape resulting from the combination of multiple dipolar couplings of different magnitudes. (d,e) Multiplet patterns formed by scalar $(\mathrm{J})$ coupling to $(\mathrm{d}) \mathrm{I}=1 / 2$ and $(\mathrm{e}) \mathrm{I}=1$ nuclei.

Figure 4. (a) Perturbation of the Zeeman energy levels of a spin $I=3 / 2$ nucleus by the quadrupolar interaction, leading to a central (CT) and satellite (ST) transitions. (b) Resulting spectra showing the effect of the first-order quadrupolar interaction on the ST for $\eta_{\mathrm{Q}}=0$ and (c) expansions showing the anisotropic broadening of the CT by the secondorder quadrupolar interaction for $\eta_{\mathrm{Q}}=0$ and 1. (d) Perturbation of the Zeeman energy levels of a spin I = 1 nucleus by the quadrupolar interaction, showing the lack of a CT. Resulting spectra showing the effect of the first-order quadrupolar interaction for $\eta_{\mathrm{Q}}=0$ and 1. 
Figure 5. (a) Schematic depiction of the MAS experiment, in which a polycrystalline sample is rotated about an axis inclined at the magic angle, $\theta_{\mathrm{m}}$ of $54.736^{\circ}$ to $\mathrm{B}_{0}$. (b) Effect of MAS on the 9.4 $\mathrm{T}{ }^{31} \mathrm{P}(\mathrm{I}=1 / 2) \mathrm{NMR}$ spectrum of $\left(\mathrm{NH}_{4}\right)_{2} \mathrm{HPO}_{4}$, showing how the anisotropic lineshape is broken up into a series of spinning sidebands which decrease in intensity as the MAS rate increases. (c) ${ }^{23} \mathrm{Na}(14.1 \mathrm{~T})$ two-dimensional MQMAS spectrum of $\mathrm{Na}_{4} \mathrm{P}_{2} \mathrm{O}_{7}$, showing a high-resolution spectrum and the four individual quadrupolar lineshapes.

Figure 6. (a) ${ }^{89} \mathrm{Y}$ MAS NMR spectra of $\mathrm{Y}_{2} \mathrm{Sn}_{2} \mathrm{O}_{7}, \mathrm{Y}_{2} \mathrm{SnTiO}_{7}$ and $\mathrm{Y}_{2} \mathrm{Zr}_{2} \mathrm{O}_{7}$, showing the effects of disorder. $\mathrm{Y}_{2} \mathrm{Sn}_{2} \mathrm{O}_{7}$ adopts an ordered pyrochlore structure, $\mathrm{Y}_{2} \mathrm{SnTiO}_{7}$ pyrochlore contains B-site cation disorder, while $\mathrm{Y}_{2} \mathrm{Zr}_{2} \mathrm{O}_{7}$ exhibits both cation and anion disorder. (b) ${ }^{27} \mathrm{Al}(14.1$ T) MAS and MQMAS NMR spectra of $\gamma-\mathrm{Al}_{2} \mathrm{O}_{3}$, with the resonances attributed to $\mathrm{Al}(\mathrm{IV})$ and $\mathrm{Al}(\mathrm{VI})$ species exhibiting lineshapes characteristic of a distribution of both chemical shifts and quadrupolar parameters.

Figure 7. (a) ${ }^{29} \mathrm{Si} /{ }^{29} \mathrm{Si}$ double-quantum (INADEQUATE) correlation spectrum of a surfactant-templated layered silicate, where the shape of the cross peaks indicates correlated disorder. Adapted with permission from Ref. [57]. Copyright (2008) American Chemical Society. (b) ${ }^{89} \mathrm{Y}$ MAS NMR spectrum of $\mathrm{Y}_{2} \mathrm{Zr}_{2} \mathrm{O}_{7}$, and values of $\Omega$ (span), extracted from amplified PASS spectra, at various points across the lineshapes. (c) ${ }^{29} \mathrm{Si}$ Jresolved spectrum of $\left({ }^{29} \mathrm{Si}\right.$-enriched) $\mathrm{SiO}_{2}$, showing how the J coupling varies across the lineshapes. Reproduced from Ref. [58]. Copyright (C) 2010 Elsevier Masson SAS. All rights reserved.

Figure 8. Schematic showing the sensitivity of NMR to dynamics over a range of timescales. The types of experiments able to measure motion on particular timescales, and the NMR parameters that are affected are shown.

Figure 9. (a) Model cluster of an aluminophosphate framework, centred on $\mathrm{P}$ with the $\mathrm{AlO}_{4}$ tetrahedra terminated by $\mathrm{H}$. (b) Schematic of a two-dimensional structure recreated 
from a unit cell containing one dopant ion, giving a formula of $\mathrm{A}_{0.75} \mathrm{~B}_{0.25}$. (c) Schematic supercell $(2 \times 2)$ that also includes one dopant ion, given a formula $\mathrm{A}_{0.9375} \mathrm{~B}_{0.0625}$.

Figure 10. (a) Schematic of the AIRSS procedure for the hydration of wadsleyite $(\beta$ $\left.\mathrm{Mg}_{2} \mathrm{SiO}_{4}\right)$, showing the removal of $\mathrm{a} \mathrm{Mg}^{2+}$ cation and the random insertion of two $\mathrm{H}^{+}$ before DFT optimisation (only an expansion of a small part of the structure is shown). (b) Enthalpy ranking of 800 AIRSS-generated structures and examples of the structural motifs observed. (c) Plot showing the variation in the calculated ${ }^{1} \mathrm{H}$ chemical shift, $\delta_{\text {iso }}$ and the ${ }^{2} \mathrm{HC}_{\mathrm{Q}}$ with changes in the hydrogen bonding arrangement around the $\mathrm{H}$. The low enthalpy structures are shown by filled circles. Reproduced (in part) from Ref. [93] with permission of the PCCP Owner Societies.

Figure 11. (a) ${ }^{89} \mathrm{Y}$ and ${ }^{119} \mathrm{Sn}$ MAS NMR spectra of $\mathrm{Y}_{2} \mathrm{Ti}_{2-\mathrm{x}} \mathrm{Sn}_{\mathrm{x}} \mathrm{O}_{7}$ pyrochlores, and the local environments of the $\mathrm{A}(\mathrm{Y}$, green) and $\mathrm{B}(\mathrm{Sn} / \mathrm{Ti}$, blue) cations within the pyrochlore structure (O atoms shown in red). (b) Plots showing calculated ${ }^{89} \mathrm{Y}$ and ${ }^{119} \mathrm{Sn}$ isotropic chemical shifts as a function of the number of Sn NNN for different arrangements of the six surrounding B site cations. Adapted with permission from Ref. [82]. Copyright (2009) American Chemical Society. Reproduced (in part) from Ref. [84] with permission of the PCCP Owner Societies.

Figure 12. ${ }^{23} \mathrm{Na} 9.4 \mathrm{~T}$ (a) MAS and (b) MQMAS NMR spectra of two samples of $\mathrm{NaNbO}_{3}$, prepared using different synthetic methods. (c) Crystal structures reported for the $\mathrm{Pbcm}$ and $P 2_{1} m a$ polymorphs of $\mathrm{NaNbO}_{3}$. Green spheres represent the sodium atoms, and the $\mathrm{NbO}_{6}$ octahedra are shown in blue. Adapted with permission from Ref. [48]. Copyright (2010) American Chemical Society.

Figure 13. (a) Variable-temperature ${ }^{17} \mathrm{O}(11.7 \mathrm{~T})$ MAS NMR spectra and two-dimensional (EXSY) spectrum $\left(57^{\circ} \mathrm{C}\right.$ ) of ${ }^{17} \mathrm{O}$-enriched $\mathrm{ZrW}_{2} \mathrm{O}_{8}$. (b) Schematic diagrams showing two possible mechanisms of oxygen exchange. Adapted from Ref. [112] with permission from with permission from John Wiley and Sons. 
Figure 14. (a) Results from Monte Carlo simulations of configurations of atoms in neighbouring tetrahedral and octahedral sheets of phlogopite (100 K), showing the correlated clustering of $\mathrm{Al}$ atoms, and ${ }^{1} \mathrm{H} /{ }^{29} \mathrm{Si}$ two-dimensional (HETCOR) correlation spectrum of phlogopite (with ${ }^{29}$ Si cross sections). Adapted from Ref. [128] with permission from the Mineralogical Society of America. (b) ${ }^{27} \mathrm{Al}$ MAS NMR spectra of kaolinite materials calcined at the temperatures indicated. Adapted with permission from Ref. [133]. Copyright (1999) American Chemical Society.

Figure 15. (a) Result of a molecular dynamics simulation of a $30 \mathrm{keV}$ recoil event in zircon.[137] (b) ${ }^{29}$ Si MAS NMR spectra of an undamaged zircon (lower) and a moderately radiation damaged zircon (upper). Adapted from Ref. [136], with the permission of AIP Publishing. (c) ${ }^{17} \mathrm{O}(11.7 \mathrm{~T})$ natural-abundance (RAPT-CPMG) MAS NMR spectra of a partially metamict zircon, highlighting the bridging (BO) and non-bridging (NBO) oxygen species. Reprinted from Ref. [140] with permission from Elsevier.

Figure 16. (a) Expansion of the structure around the $\mathrm{OH}$ sites in clinohumite and fluorinated clinohumite. (b,c) ${ }^{17} \mathrm{O}$ experimental and calculated (b) MQMAS and (c) STMAS NMR spectra of clinohumite. In (b), simulated spectra result both from the summation of ${ }^{17} \mathrm{O}$ isotropic spectra calculated for each oxygen within the unit cell for different proton arrangements, and from the mean value of the calculated isotropic shifts. In (c), spectra were simulated using the rate constants for $\mathrm{H} 1-\mathrm{H} 2$ exchange given. (d) Variabletemperature rotor-synchronised ${ }^{2} \mathrm{H}$ MAS NMR spectra of deuterated clinohumite and Arrhenius plot of the natural logarithm of the linewidth against $1 / T$. (e) ${ }^{19}$ F MAS NMR spectrum of fluorinated clinohumite, and calculated ${ }^{19} \mathrm{~F}$ chemical shifts. (a,e) Adapted with permission from Ref. [86]. Copyright (2010) American Chemical Society. (b,c) Adapted with permission from Ref. [146]. Copyright (2009) American Chemical Society. (d) Reproduced from Ref. [147] with permission from the PCCP Owner Societies. 
Figure 17. (a) Experimental (left) and simulated (right) wideline ${ }^{2} \mathrm{H}$ NMR spectra of $\mathrm{C}_{2} \mathrm{D}_{4}$ adsorbed on Ag/H-ZSM-5. Simulated spectra were produced based on a model of ethylene rotation about $\mathrm{C}_{2}$ and $\mathrm{C}_{2}$ ' axes by four-site exchange. (b) Arrhenius plots for the rate constants $\mathrm{k}_{1}$ (circles) and $\mathrm{k}_{2}$ (squares) for $\mathrm{C}_{2} \mathrm{D}_{4}$ adsorbed on Ag/H-ZSM-5. (c) Types of intramolecular rotations of ethylene when forming a $\pi$-complex with $\mathrm{Ag}^{+}$in $\mathrm{Ag} / \mathrm{H}-\mathrm{ZSM}-5$. Adapted with permission from Ref. [167]. Copyright (2016) American Chemical Society.

Figure 18. (a) Unit cell of dehydrated STA-2(BDAB) showing the six possible $\mathrm{OH}^{-}$ locations considered. (b) Experimental (right) ${ }^{27} \mathrm{Al} /{ }^{31} \mathrm{P}$ two-dimensional correlation spectrum (INEPT, $20.0 \mathrm{~T}$ ) and calculated (left) cross-peak positions for STA-2(BDAB) for models with different $\mathrm{OH}^{-}$positions. Best agreement is obtained for models where $\mathrm{OH}^{-}$ groups occupy different cancrinite cages and sit on Al species that do not share a D4R (blue/green points). Ref. [174] - Published by The Royal Society of Chemistry. (c) ${ }^{13} \mathrm{C} \mathrm{CP}$ MAS spectra of SIZ-4 prepared with bmim and emim as the SDA. When bmim is used an ordered AlPO is formed with dmim incorporated into the pores after a transalkylation reaction, while the use of emim leads to both dmim and emim in the pores in a more disordered material.[182] (d) ${ }^{31} \mathrm{P}$ MAS NMR spectra of calcined AlPO-34 left under ambient conditions for 0-14 days.[187,188]

Figure 19. (a) ${ }^{29} \mathrm{Si} \mathrm{CP}$ MAS NMR spectra of a CHA-type SAPOs with varying Si/ Al ratio. Adapted with permission from Ref. [192]. Copyright (2007) American Chemical Society. (b) ${ }^{31} \mathrm{P}$ MAT spectrum of MgAPO-20, with lineshapes extracted at varying positions in $\delta_{1}$. Adapted with permission from Ref. [194]. Copyright (1998) American Chemical Society.

Figure 20. (a) Experimental ${ }^{13} \mathrm{C}-{ }^{15} \mathrm{~N}$ REDOR measurements and MD simulations for different proportions of different types of linkers in MOF-5. Possible arrangements include large clusters (LC), small clusters (SC), random (Ran), and alternating (Alt) linkers. From Ref. [199]. Reprinted with permission from AAAS. (b) ${ }^{15}$ N CP MAS spectra of Al-MIL-101$\mathrm{NH}_{2}$ (top) and Al-MIL-101-URPh (bottom) after post-synthetic modification. (c) ${ }^{1} \mathrm{H}$ 
projections of ${ }^{1} \mathrm{H}^{27} \mathrm{Al}$ correlation (D-HMQC) spectra of Al-MIL-101- $\mathrm{NH}_{2}$ (top) and Al-MIL101-URPh (bottom), showing that there is less water spatially near the $\mathrm{Al}$ centres in the modified MOF. Reproduced from Ref. [207] with permission.

Figure 21. (a) Experimental (right) and simulated (left) ${ }^{2} \mathrm{H}$ wideline NMR spectra of fully deuterated $\mathrm{Sc}_{2}(\mathrm{bdc})_{3}$. Spectra are simulated assuming a $180^{\circ}$ flip of a deuterated benzene ring. Reprinted with permission from Ref. [208]. Copyright (2011) American Chemical Society. (b) ${ }^{13} \mathrm{C}(7.05 \mathrm{~T})$ wideline NMR spectra of ${ }^{13} \mathrm{C}$-enriched $\mathrm{CO}_{2}$ adsorbed in $\mathrm{Mg}$-CPO-27 as a function of temperature. Reprinted with permission from Ref. [215]. Copyright (2012) American Chemical Society. (c) ${ }^{17} \mathrm{O}(9.4 \mathrm{~T})$ wideline NMR spectra of ${ }^{17} \mathrm{O}$-enriched $\mathrm{CO}_{2}$ adsorbed in $\mathrm{Mg}-\mathrm{CPO}-27$ as a function of temperature. Also shown are simulated spectra, calculated to take account of a local "wobbling" about the molecular axis, in addition to the six-site exchange between equivalent binding sites. Reprinted with permission from Ref. [216]. Copyright (2014) American Chemical Society.

Figure 22. (a) ${ }^{13} \mathrm{C}$ CP MAS NMR spectra of two forms of finasteride, with one and two distinct molecules in the asymmetric unit, respectively. Reprinted from Ref. [227], with permission from Elsevier. (b) ${ }^{15} \mathrm{~N} /{ }^{15} \mathrm{~N}$ double-quantum (INADEQUATE) correlation spectra of two deoxyguanosine derivatives. Adapted with permission from Ref. [233]. Copyright (2005) American Chemical Society. (c) ${ }^{13} \mathrm{C}$ CP MAS NMR spectrum of a simulated drug formulation containing 5\% bambuterol hydrochloride (BHC). Adapted from Ref. [221] with permission from John Wiley and Sons.

Figure 23. (a) The conversion of the 10-hydroxy-10,9-boroxophenanthrene dimer into the corresponding anhydride. (b) ${ }^{13} \mathrm{C} \mathrm{CP}$ MAS NMR spectrum of the resulting anhydride. (c) ${ }^{11} \mathrm{~B}(9.4 \mathrm{~T})$ MQMAS spectrum of the boroxophenanthrene solid heated at $50{ }^{\circ} \mathrm{C}$ for $24 \mathrm{~h}$, showing the presence of signals from the boroxophenanthrene (red line) and the anhydride (green line). (d) Computational mapping of the transformation of the boroxophenanthrene into the anhydride (via proton transfer) within the crystal lattice. Reprinted from Ref. [87], with permission from Elsevier. 
Figure 24. (a) Variable-temperature $T_{1 \rho}$ measurements of octafluoronaphthalene, together with the fit used to extract the kinetic parameters. (b) Time evolution of the $\phi$ polar angle for an octafluoronaphthalene molecule during a $290 \mathrm{~K}$ MD simulation, showing jumps between four distinct orientations. (c) Potential energy surface, calculated from the MD simulations, for octafluoronaphthalene as a function of molecular orientation, defined in terms of the polar angles, $\phi$ and $\theta$. (d) An example of an octafluoronaphthalene molecule reorienting within its local crystal structure (by $\sim 40^{\circ}$ ). Adapted with permission from Ref. [251]. Copyright (2010) American Chemical Society.

Figure 25. Proposed model for bone mineral consisting of unit cells of OCP-citrate split along (200) and sandwiching unit cells of HA, resulting in a parallel arrangement of mineral platelets alternating with hydrated layers. Blue lines indicate the surfaces of the mineral platelets (defined so that all calcium ions are contained within), which are bridged by citrate anions. Reproduced from Ref. [260] with permission.

Figure 26. (a) Six distinct Se environments found in AsSe glasses and their respective contributions to the ${ }^{77}$ Se MAS NMR spectrum. Se atoms are shown in yellow and As in black. Distinct Se atoms are shown in blue, red and purple. Adapted with permission from Ref. [281]. Copyright (2015) American Chemical Society. (b) ${ }^{17} \mathrm{O}$ (9.4 T) MQMAS NMR spectra of sodium silicate glasses with varying iron content. Reprinted from Ref. [282] Copyright (2016), with permission from Elsevier. (c) Simulated ${ }^{17} \mathrm{O}$ (14.1 T) MAS and MQMAS NMR spectra for calcium silicate sodium glass structures generated using MD. Adapted with permission from Ref. [284]. Copyright (2012) American Chemical Society.

Figure 27. (a) ${ }^{13} \mathrm{C} C P$ MAS NMR spectrum of amorphous (atactic) polypropylene at $252 \mathrm{~K}$. The abbreviation describes the conformation around the carbon centre, with $t$ and $g$ denoting trans and gauche, respectively. Ref. [292] Copyright (2003) Wiley-VCH Verlag $\mathrm{GmbH} \&$ Co. KGaA. Reproduced with permission. (b) Tautomeric structures with different hydrogen bonding arrangements for ureido-pyrimidone supramolecular 
polymers, along with ${ }^{1} \mathrm{H}$ double-quantum correlation spectra that confirm the proximity of the different H species. Reproduced from Ref. [293] with permission from the PCCP Owner Societies. (c) ${ }^{19} \mathrm{~F}$ MAS NMR spectrum of a copolymer of vinylidene fluoride and trifluoroethylene, along with 'filtered' spectra, in which only the amorphous or crystalline parts of the sample are observed. Reproduced with permission from Ref. [294] Copyright (2003) Elsevier Ltd. (d) ${ }^{2} \mathrm{H}$ two-dimensional exchange spectrum showing chain motion in the crystalline phase of poly(vinylidenefluoride) at $\mathrm{T}=370 \mathrm{~K}$, with a mixing time of 200 ms. Adapted with permission from Ref. [295]. Copyright (1991) American Chemical Society. 
Figure 1

a

○००००००

○००००००

○००००००

00000000

○००००००

- 000000

○००००००

$\circ \circ \circ \circ \circ \circ \circ$

C

○ㅇํㅇำ

$\circ \circ 000$

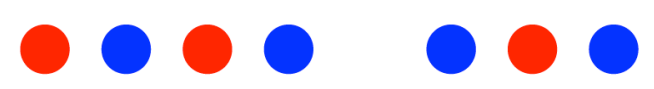

- 0000

○०००००००

- 00000

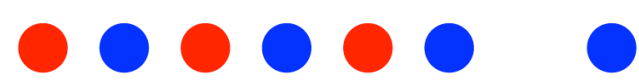

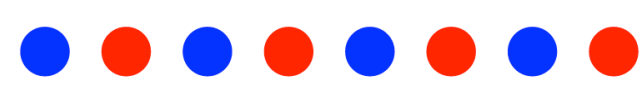

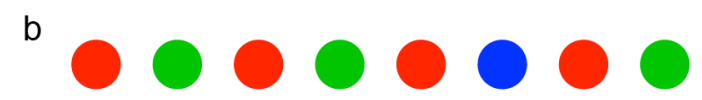

00000000

00000000

10000000

00000000

00000000

00000000

00000000

d

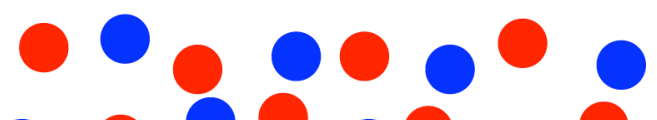

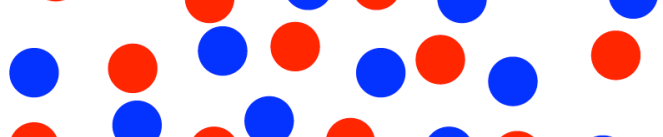

- 000000

1000000

1000000

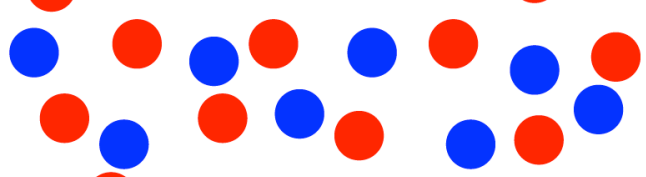

1000000 
Figure 2

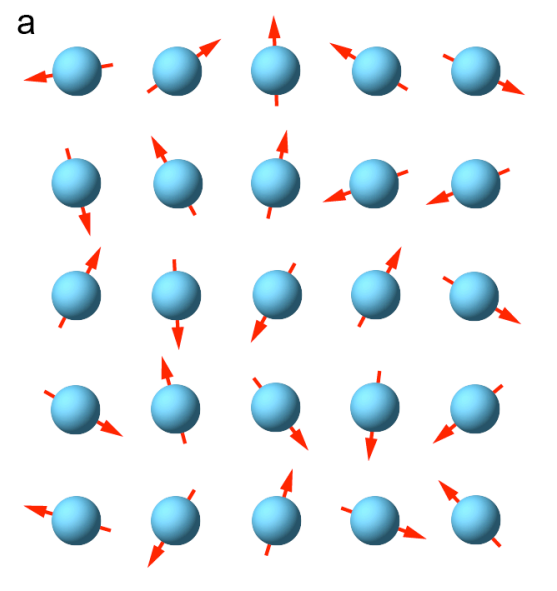

no applied field

b

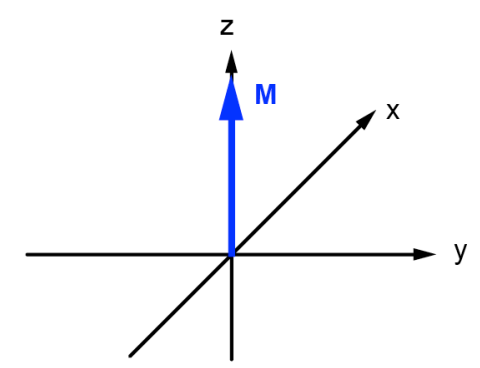

thermal equilibrium

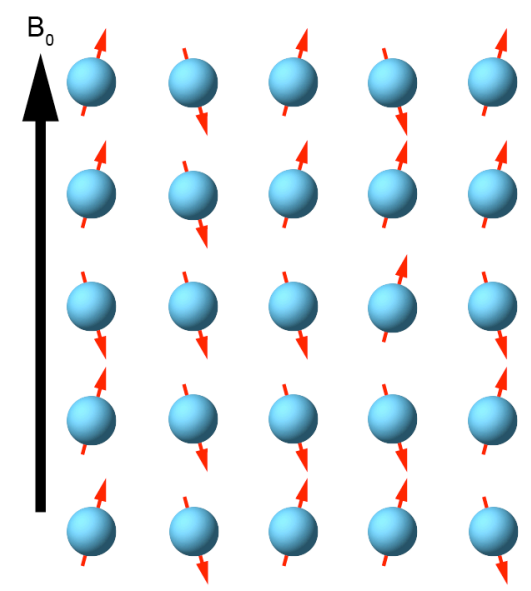

$\mathrm{B}_{0}$ field applied along $\mathrm{z}$

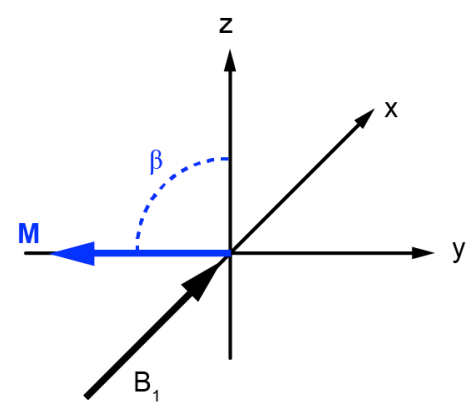

$B_{1}$ field applied along $+x$

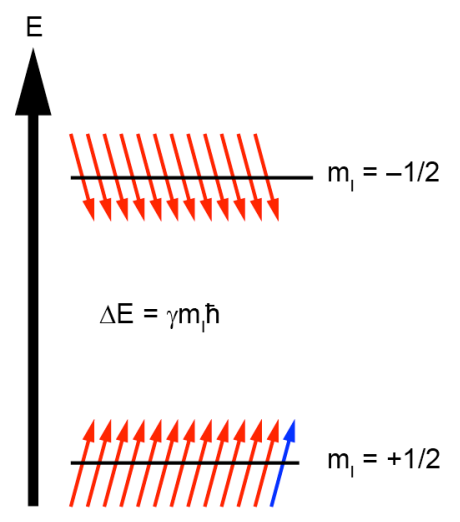

spin populations

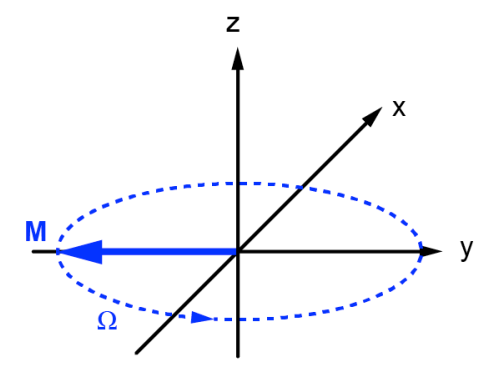

precession in the xy plane
C

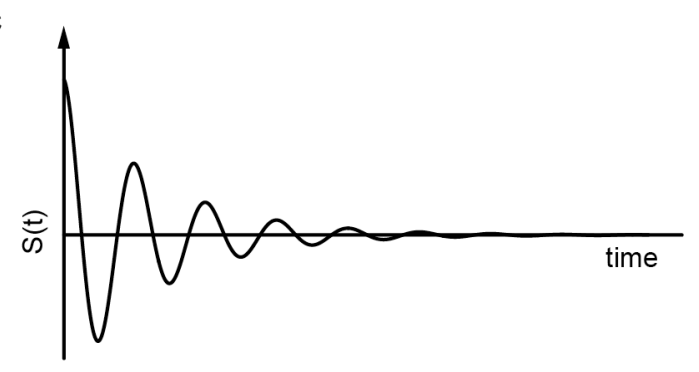

d

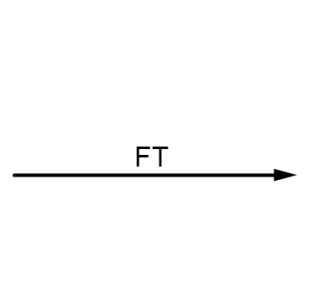

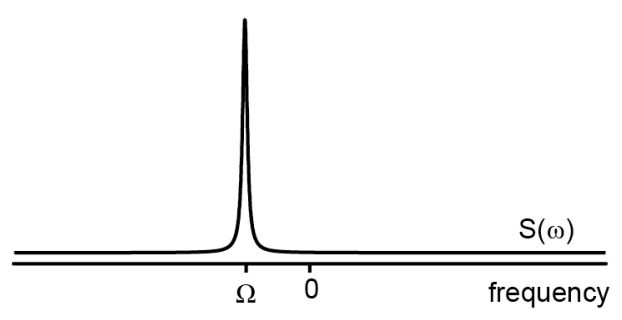


Figure 3

a
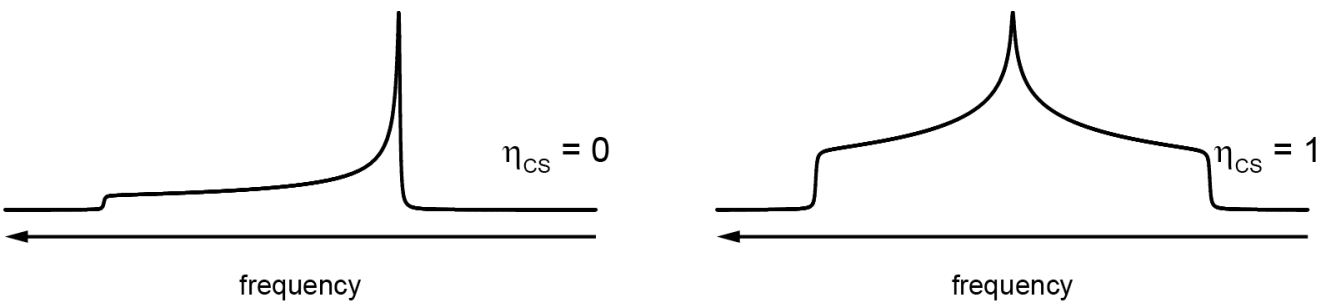

b

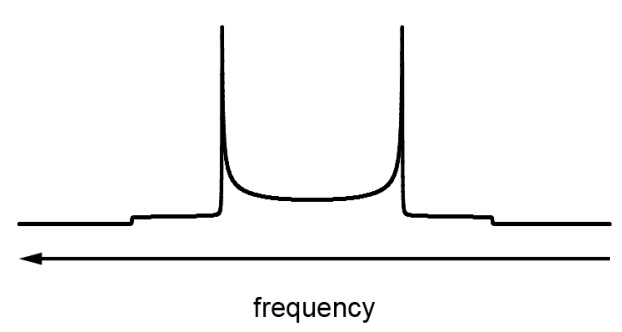

C

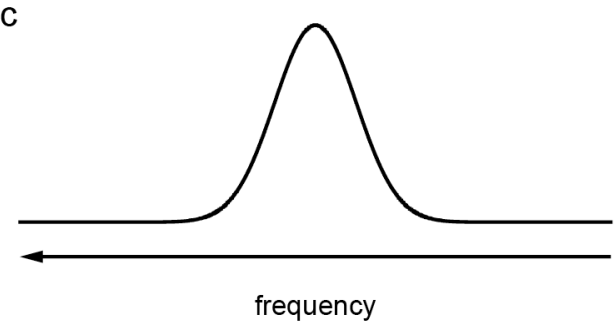

d
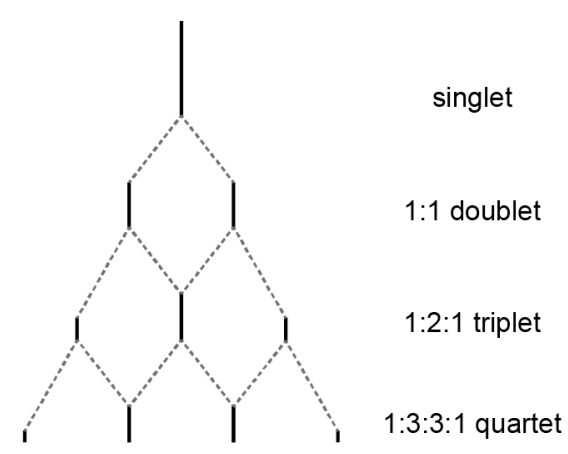

e

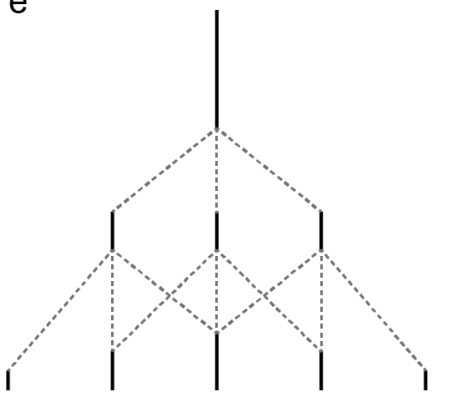

singlet

1:1:1 triplet

1:2:3:2:1 quintet 


\section{Figure 4}

a

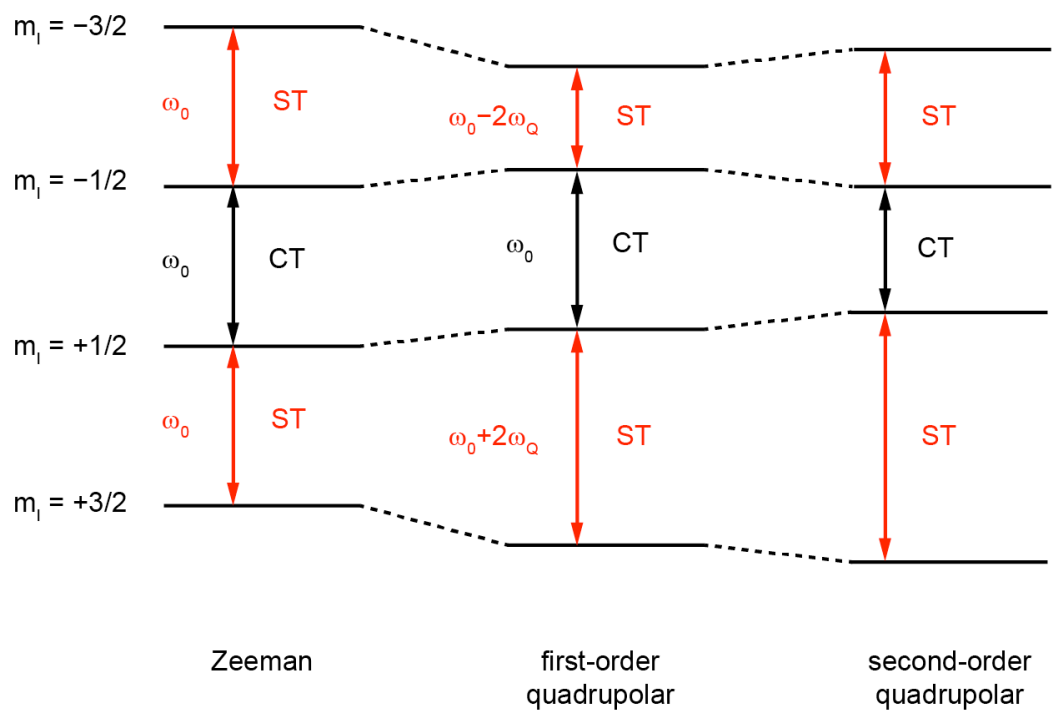

b

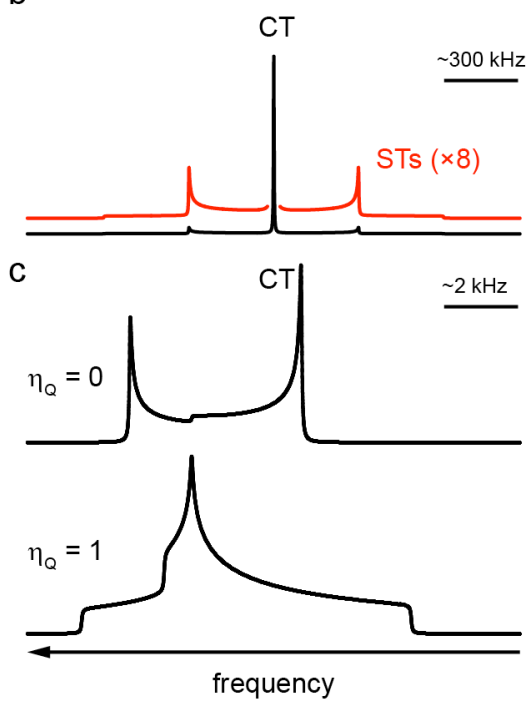

d
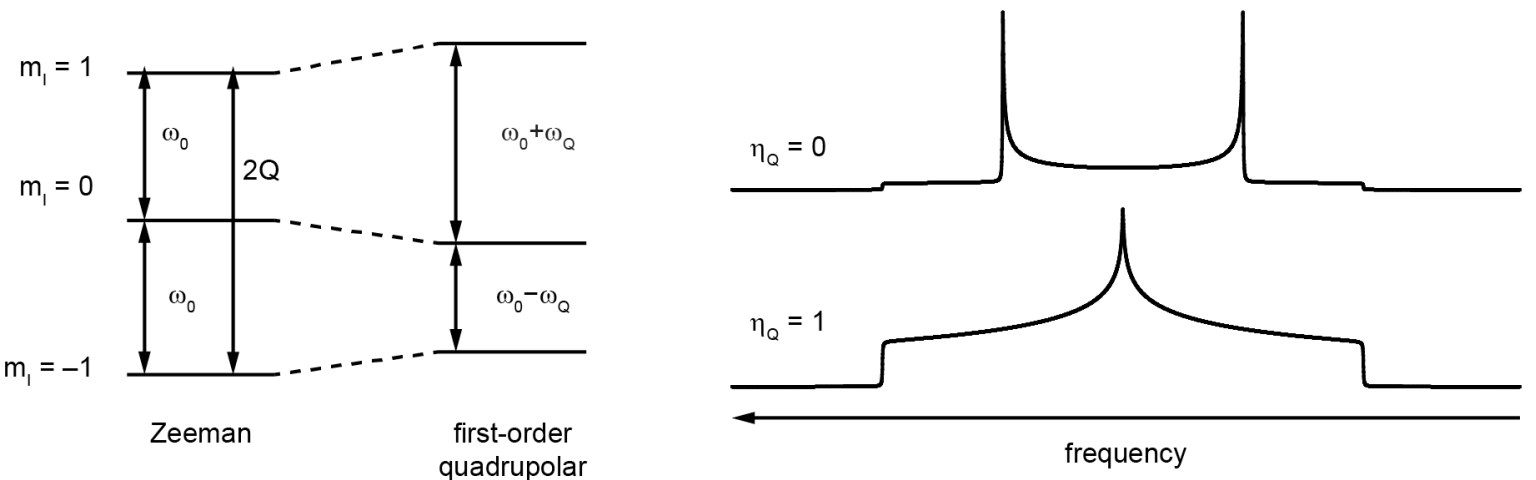
Figure 5

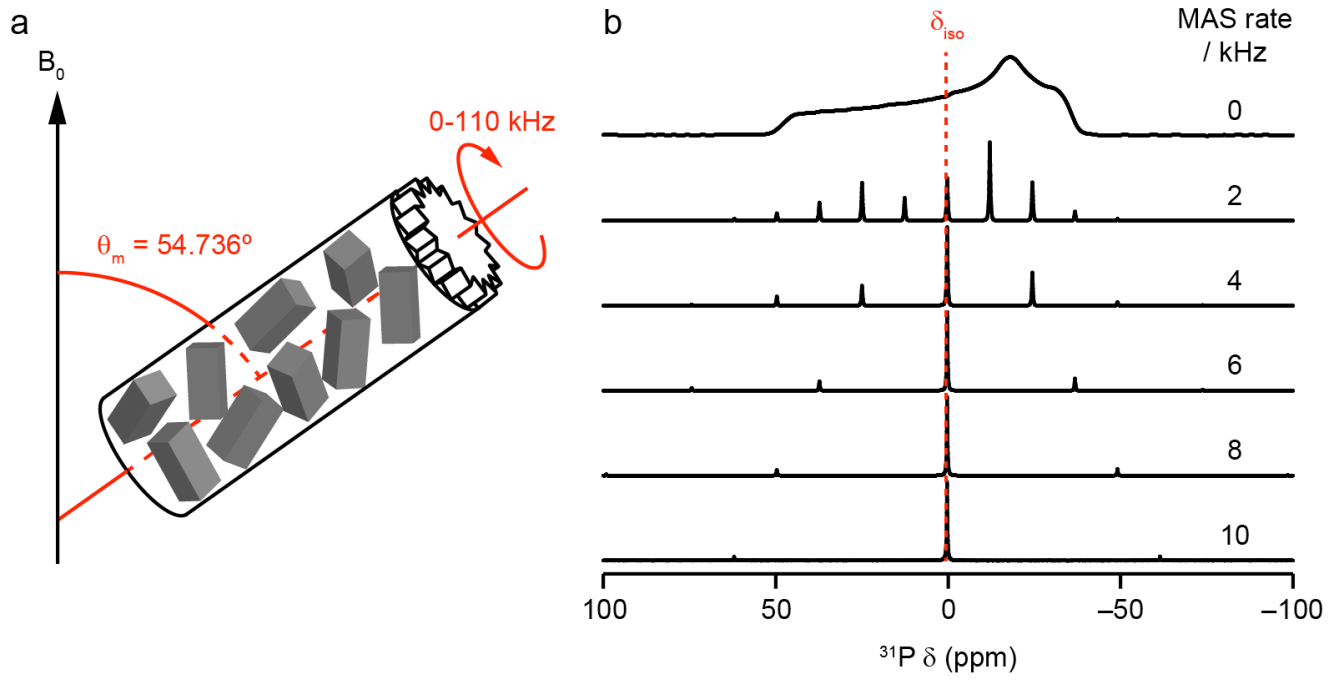

C

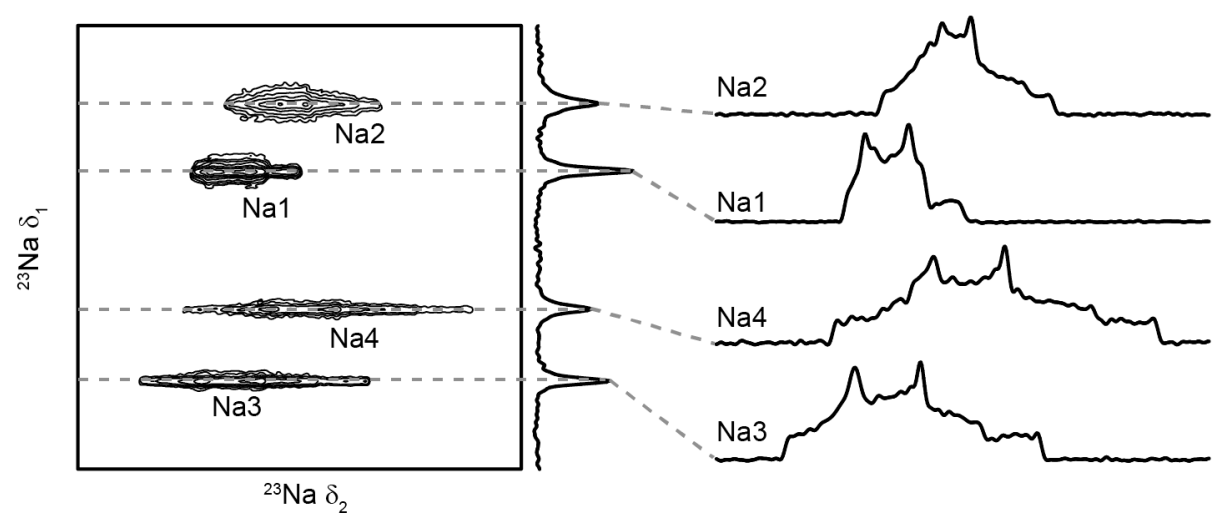


Figure 6

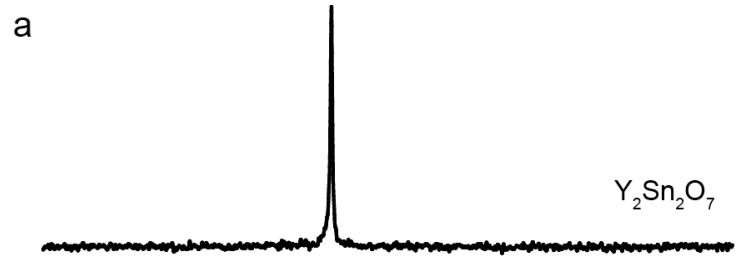

b
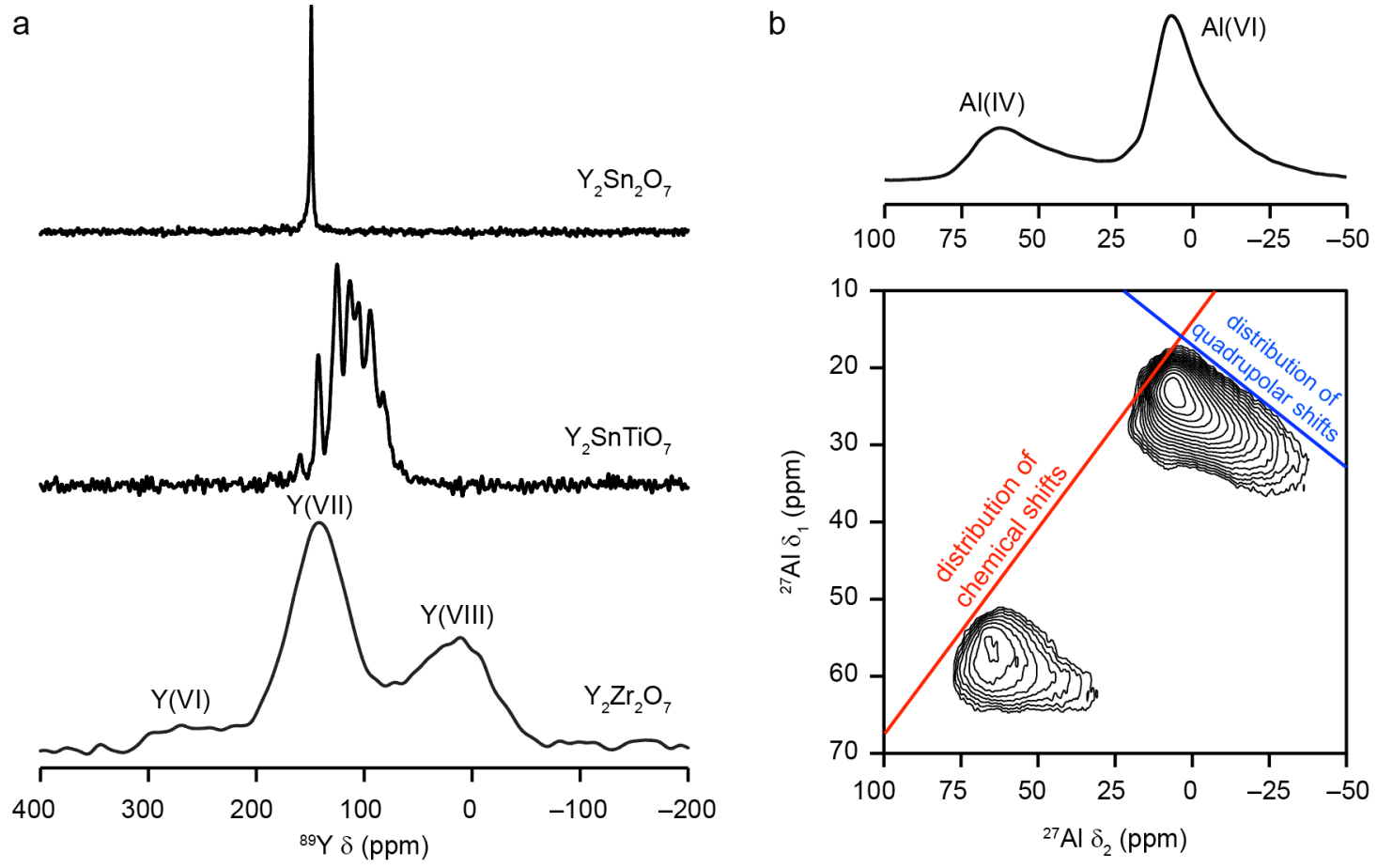
Figure 7

a

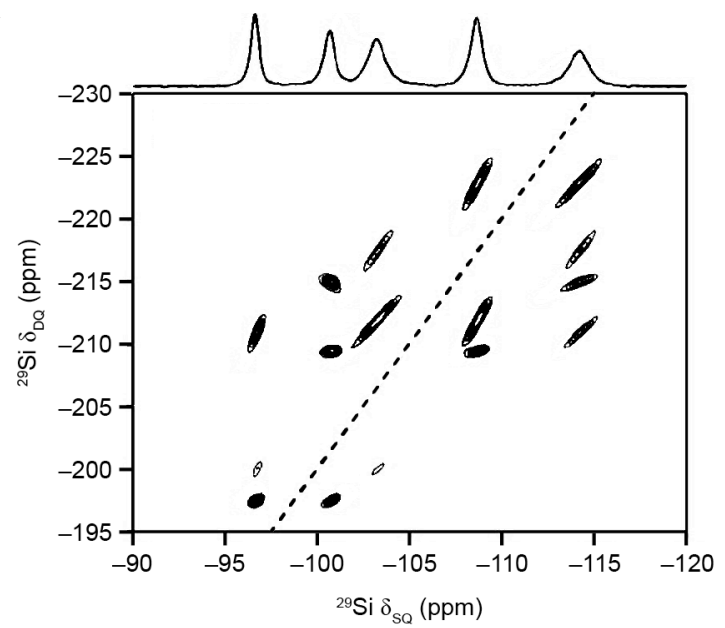

b

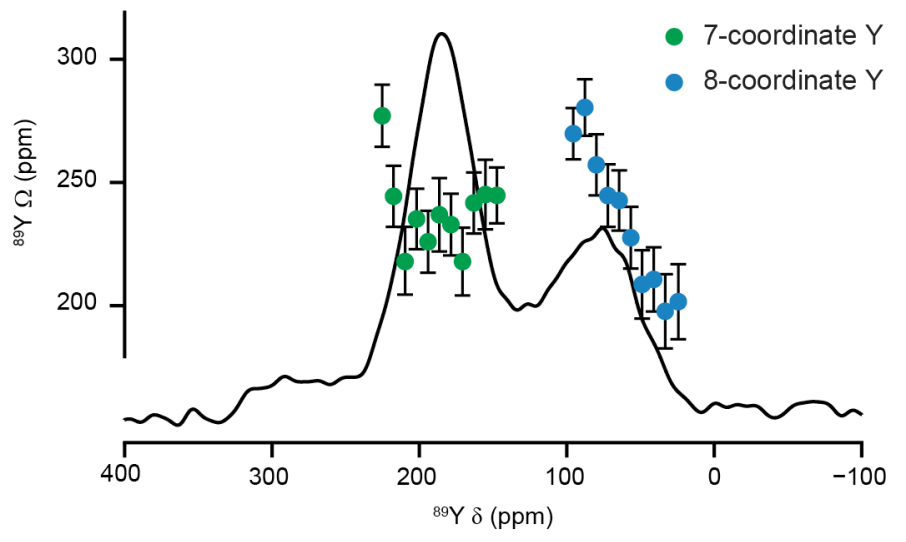

C

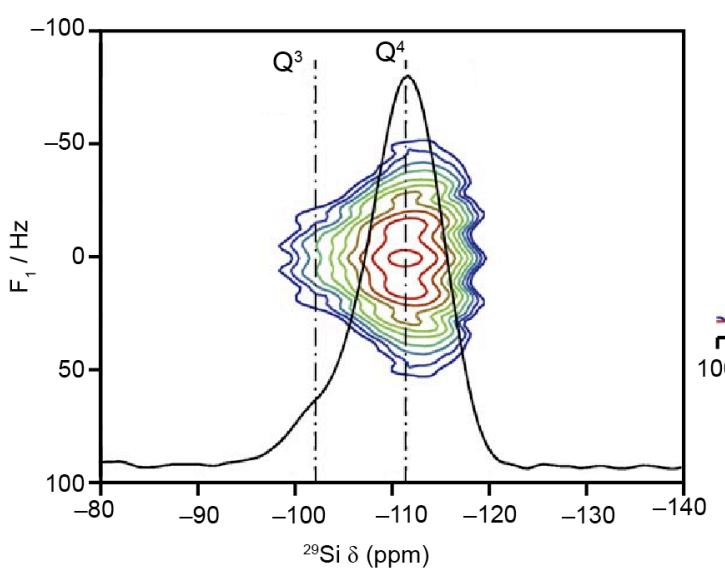

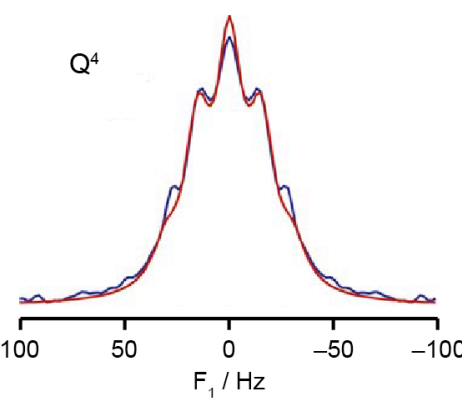

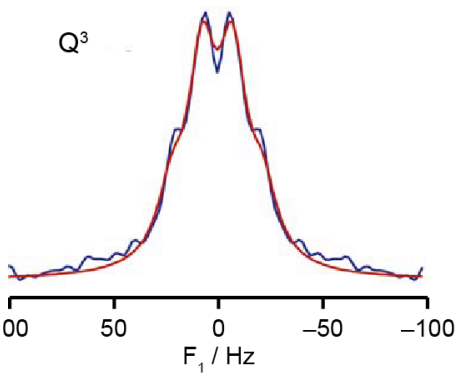


Figure 8

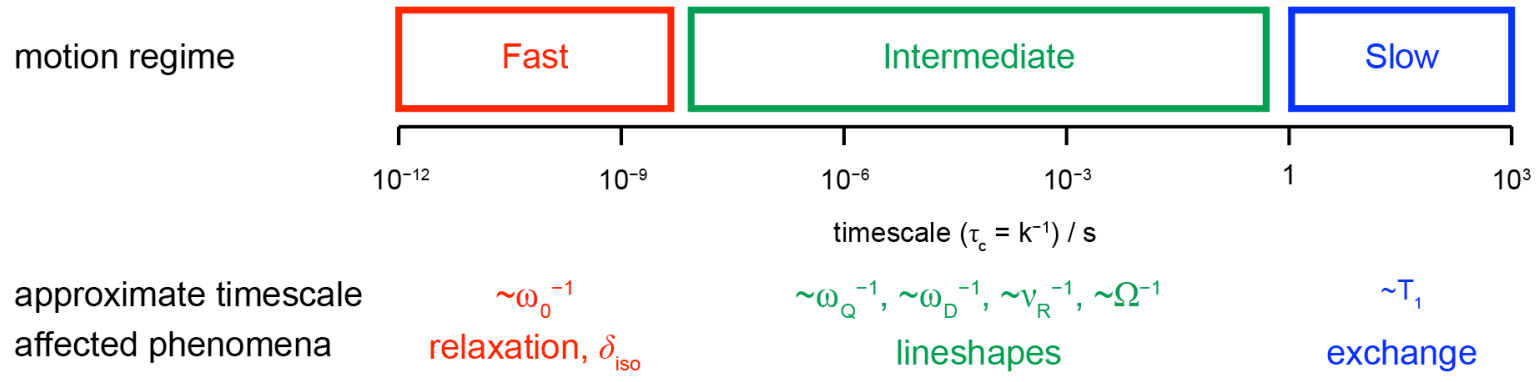
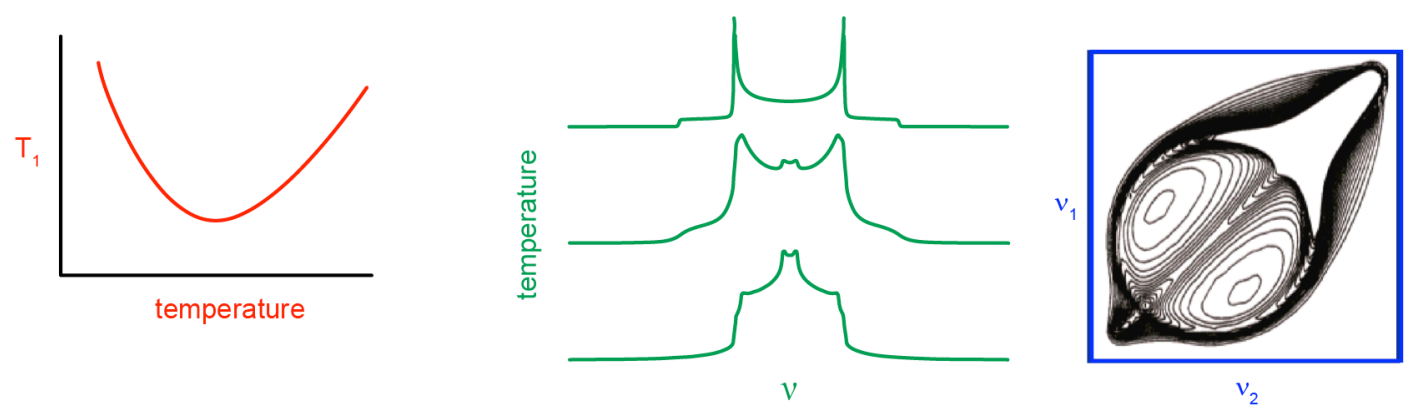
Figure 9

a

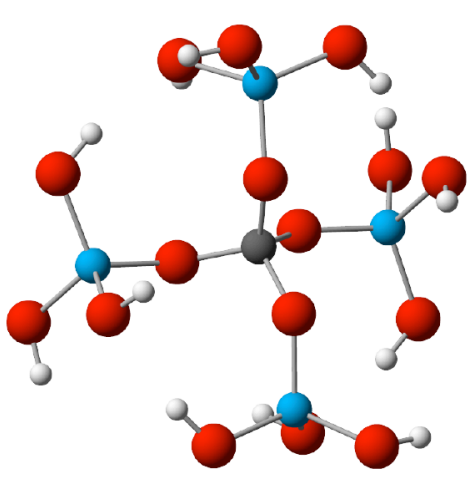

b

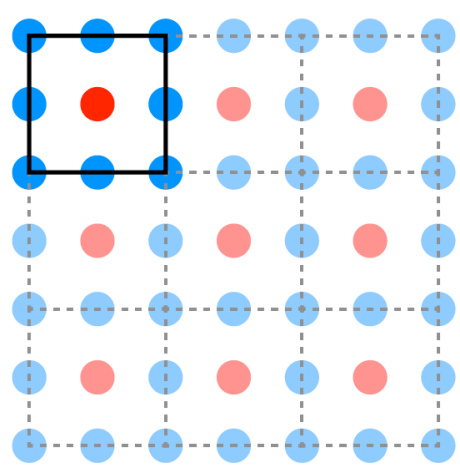

C

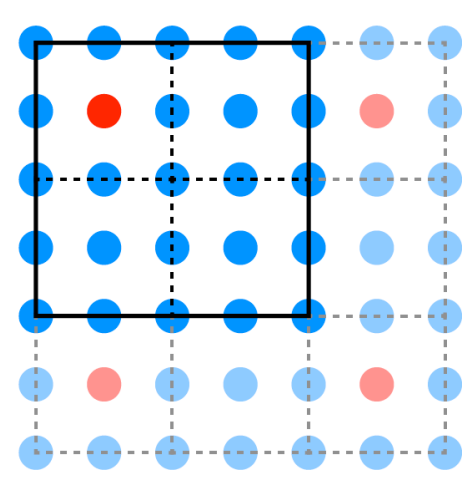


Figure 10

a

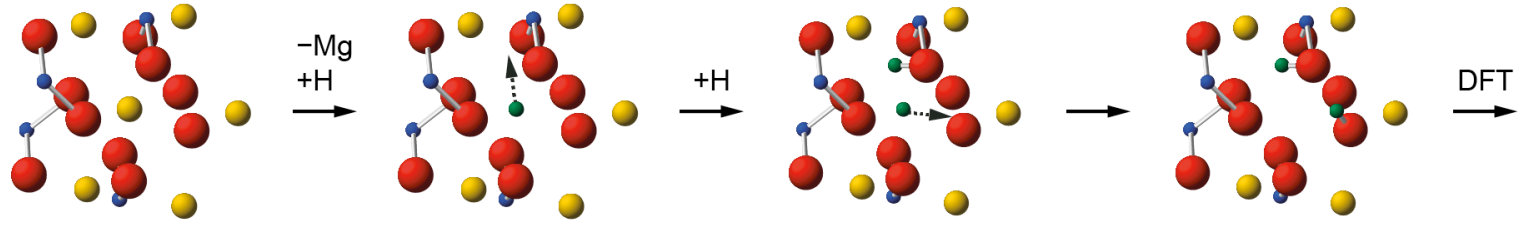
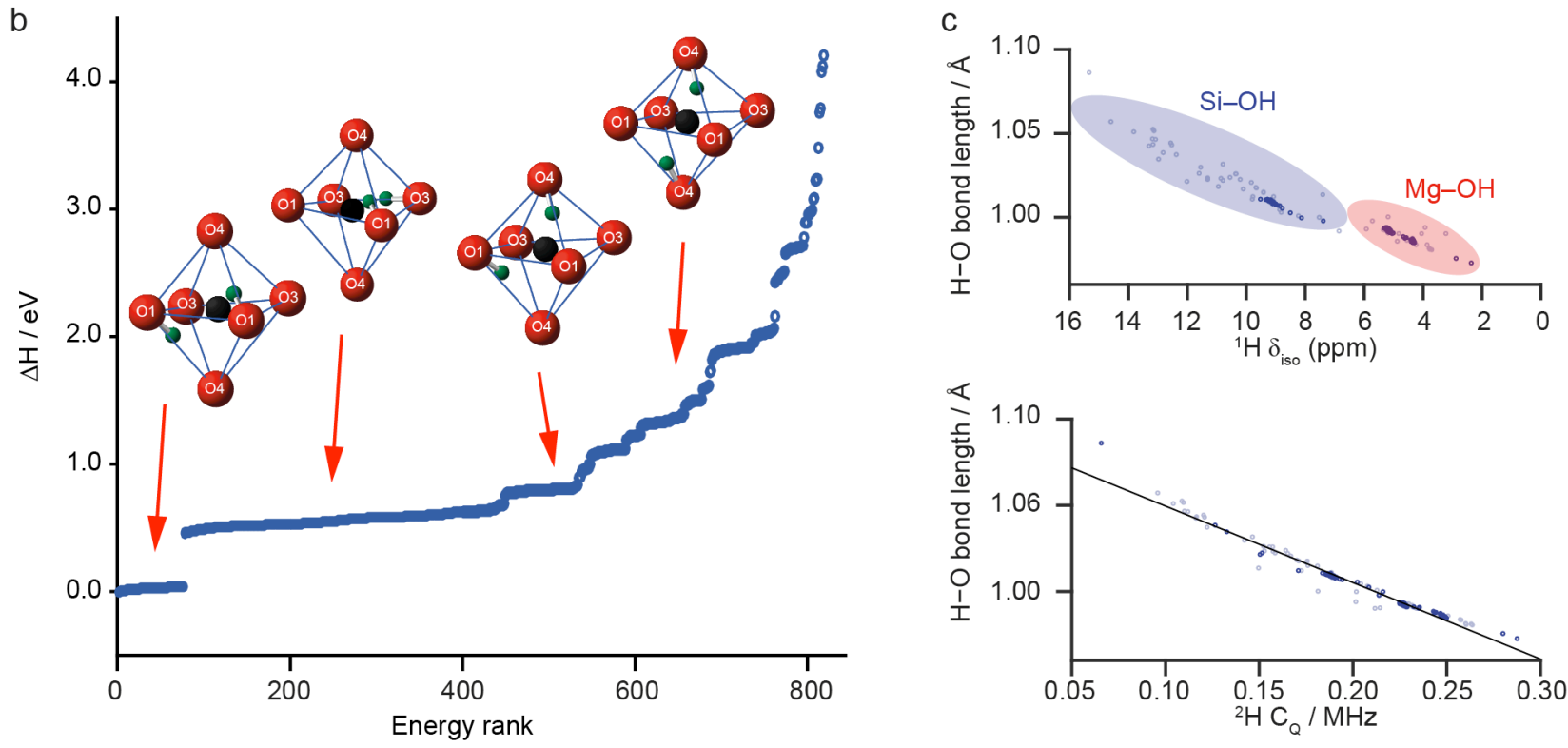
Figure 11
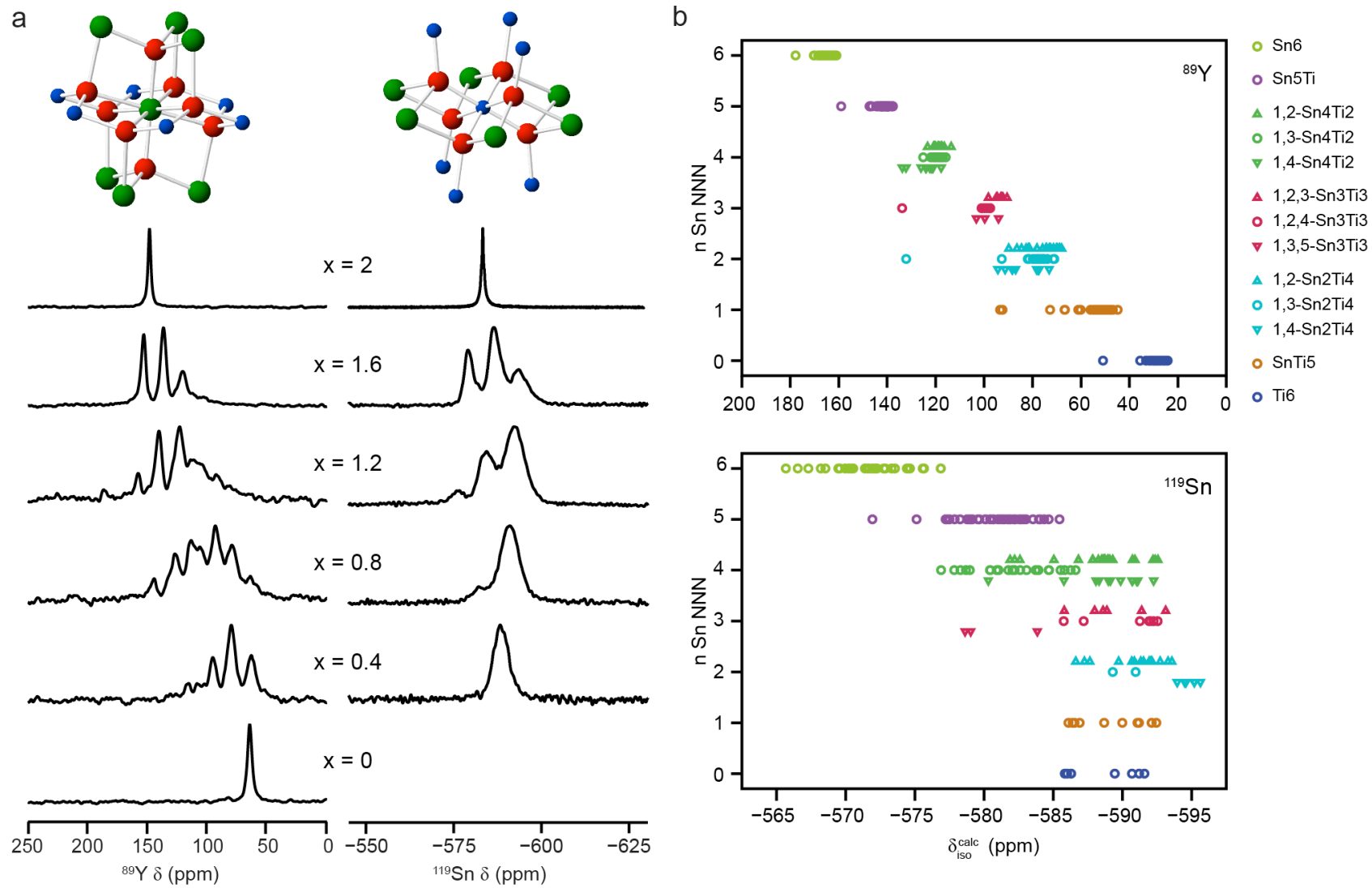
Figure 12

a

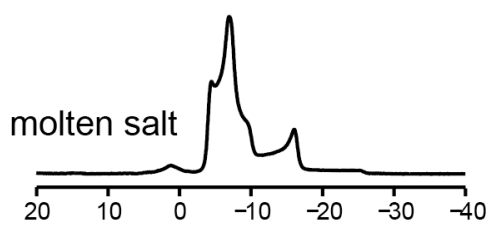

b

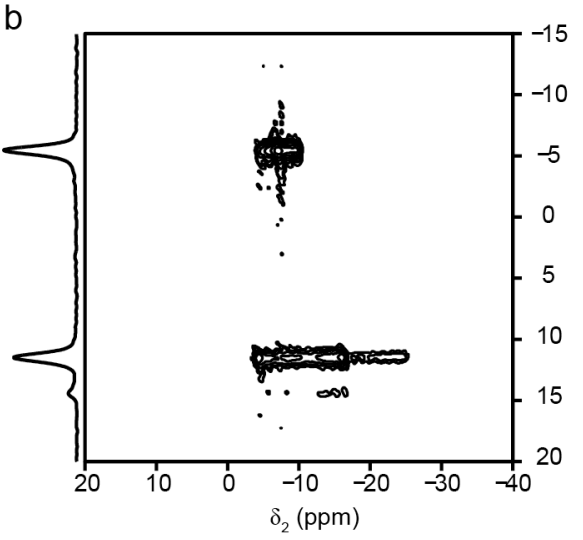

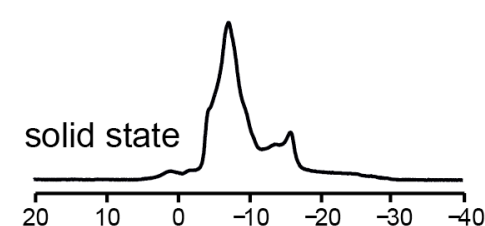

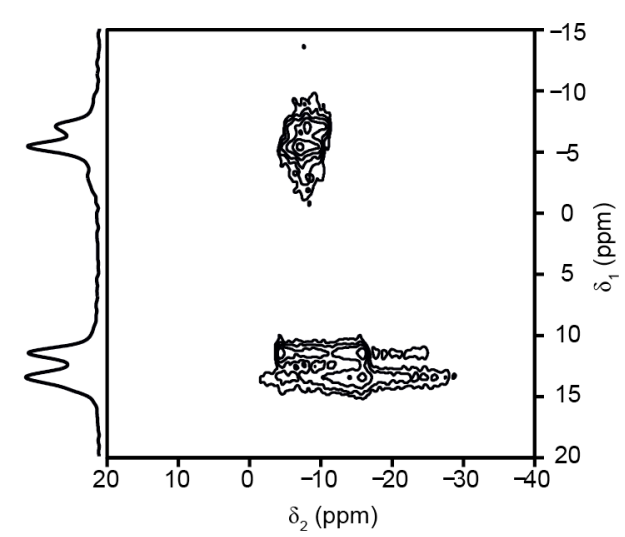

C

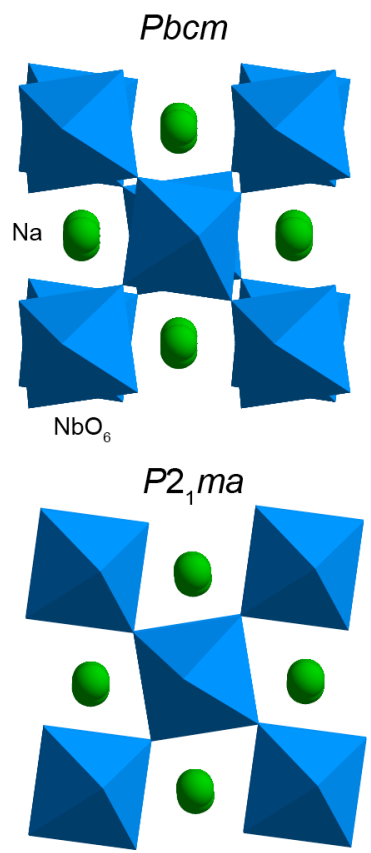


Figure 13
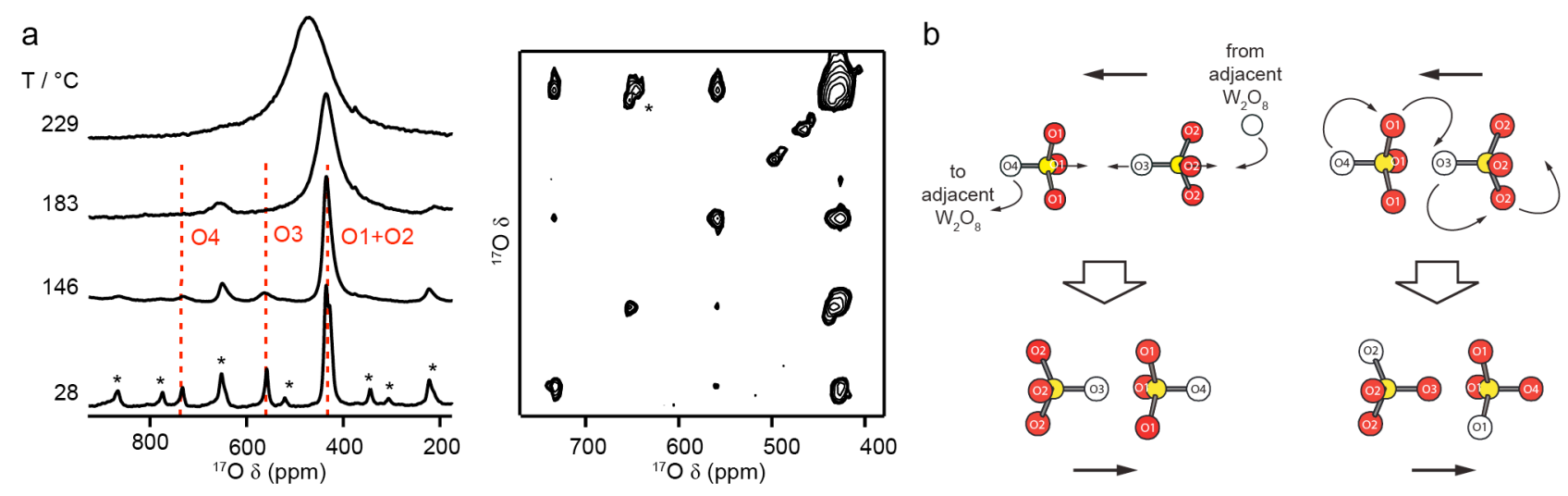
Figure 14

a
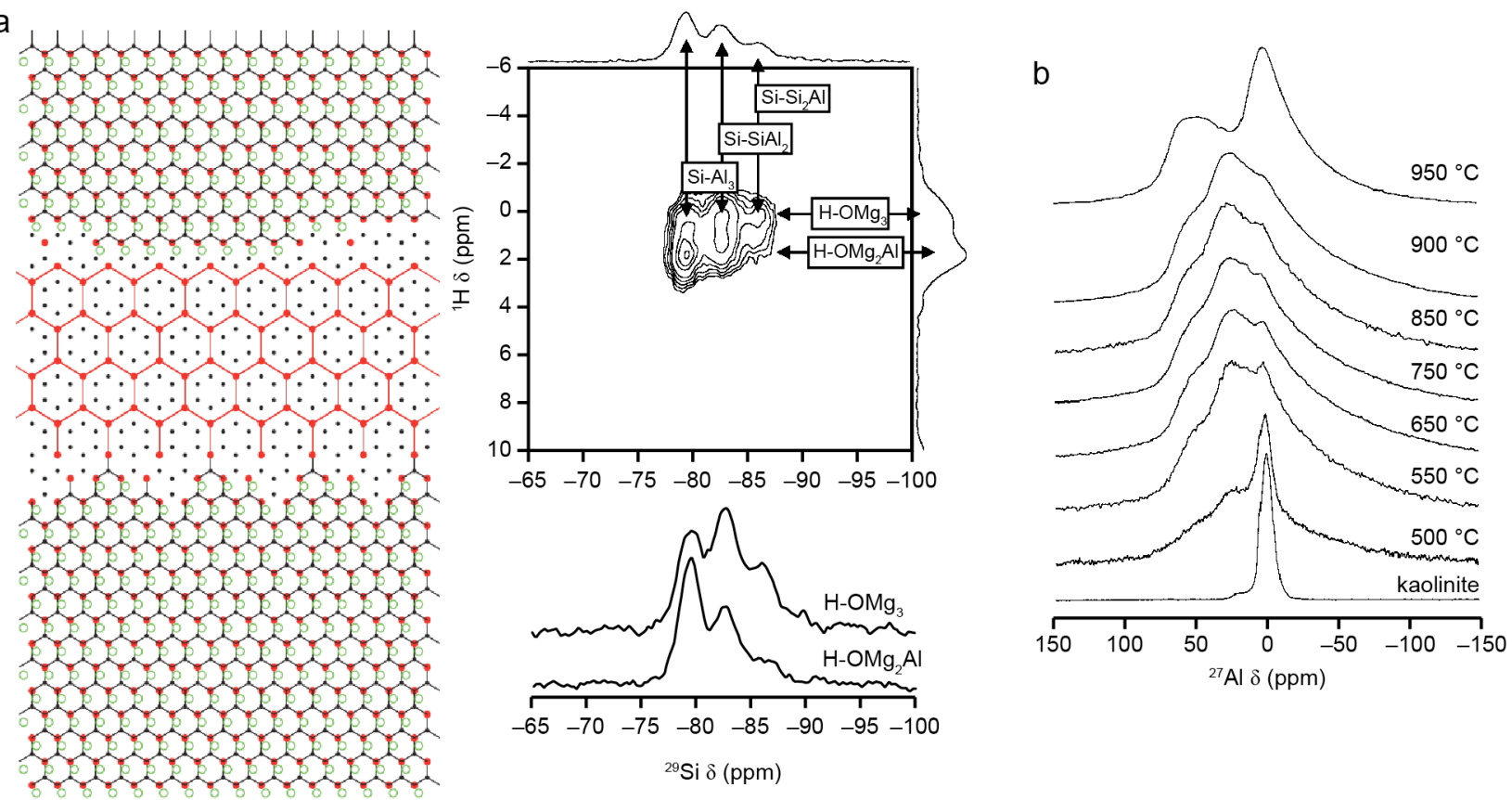
Figure 15
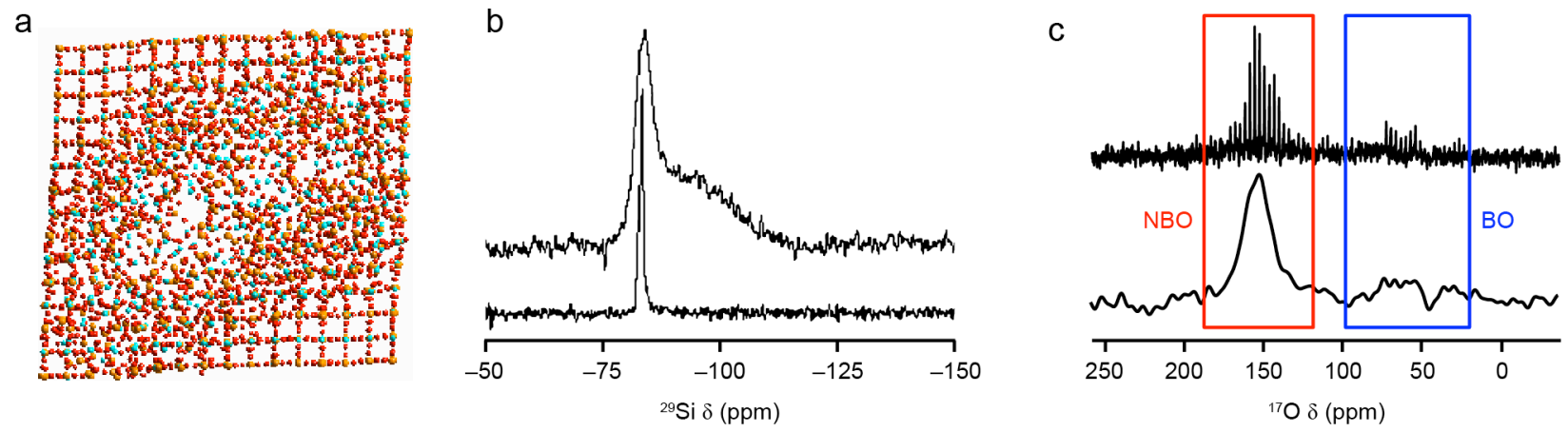
Figure 16

a
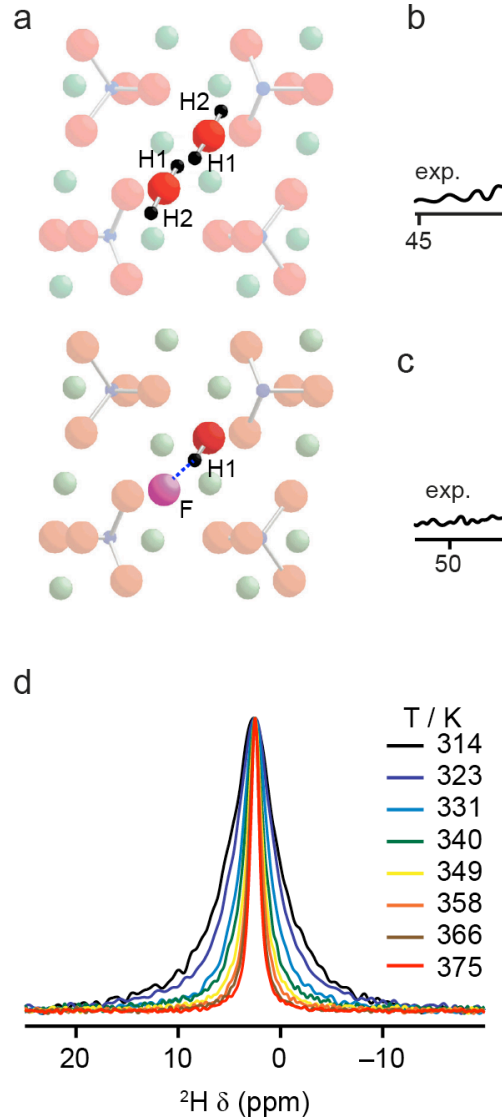

b
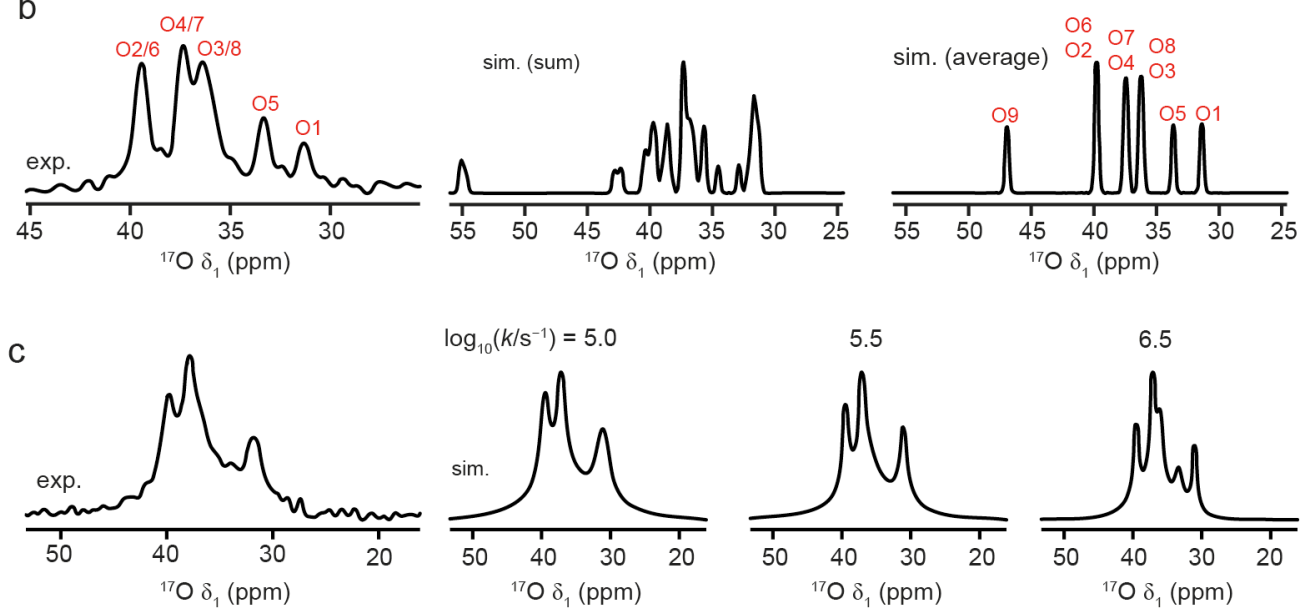

e

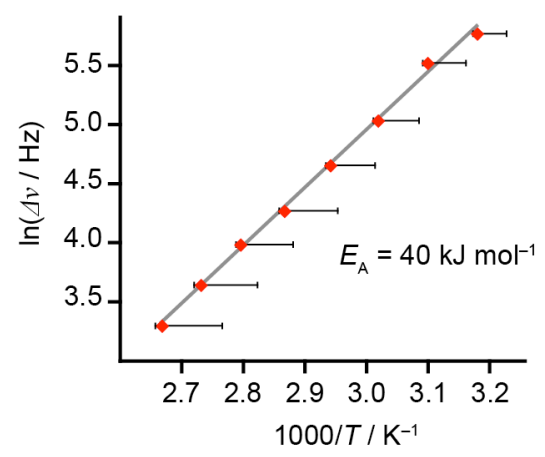




\section{Figure 17}
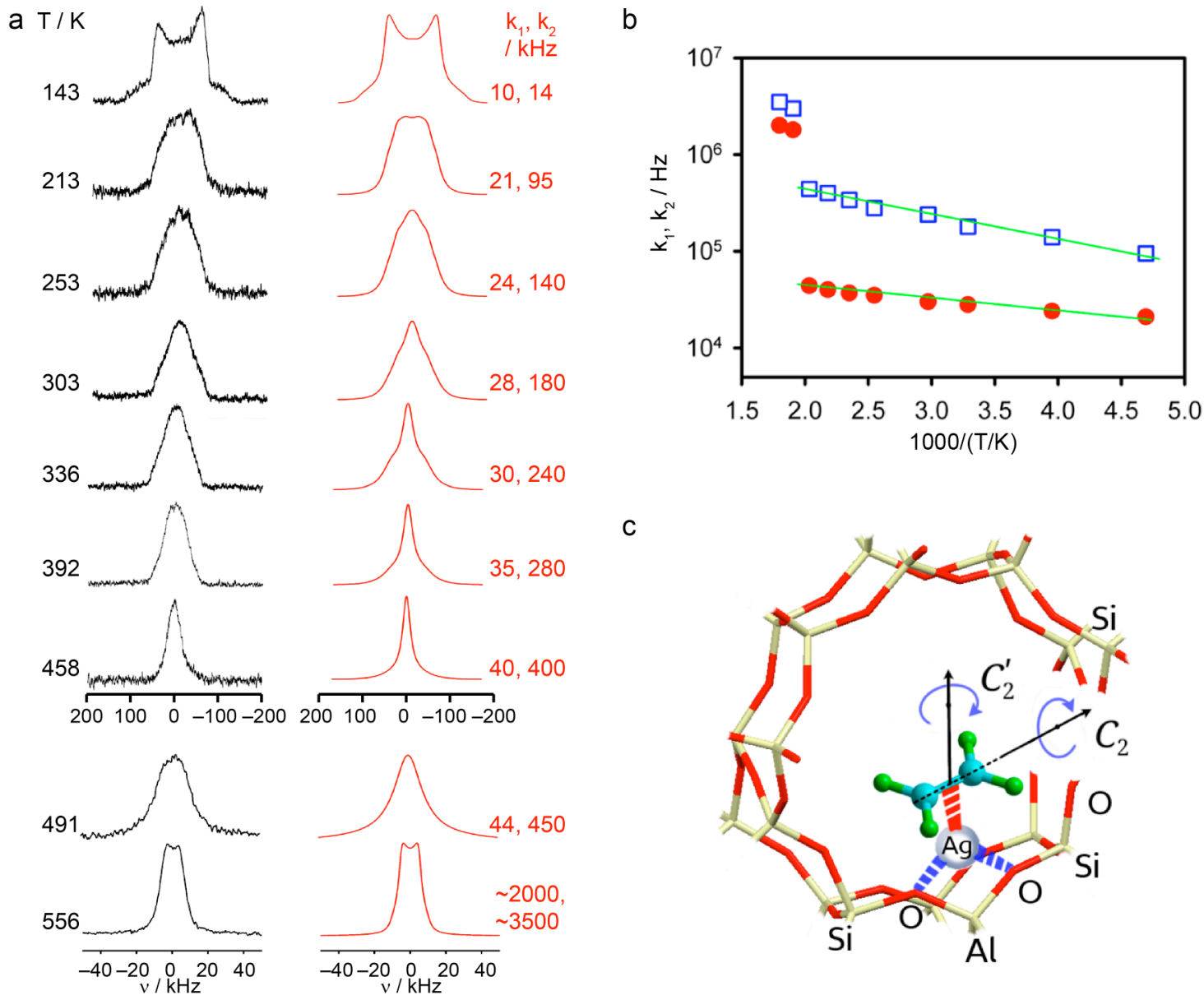

C

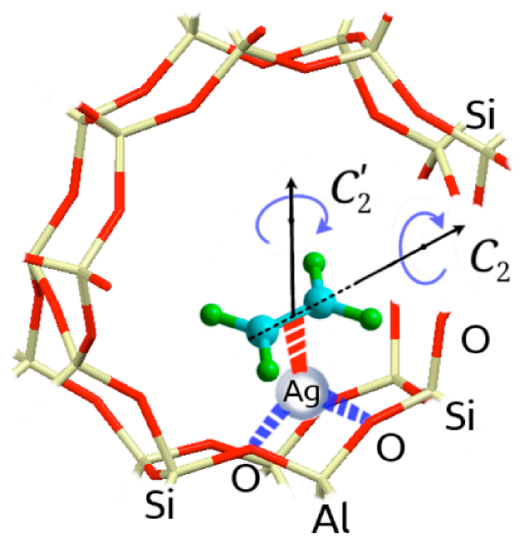


Figure 18
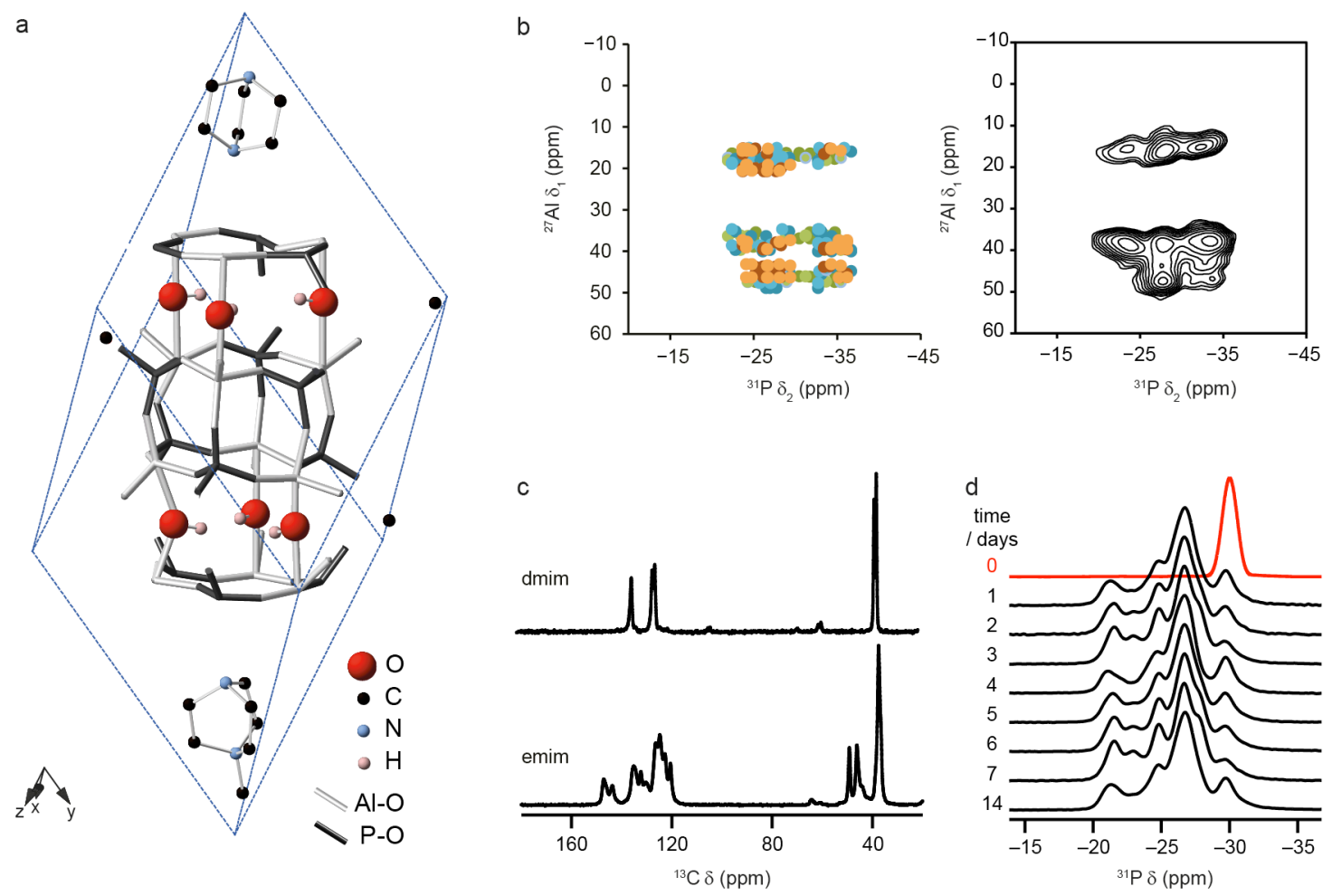
Figure 19
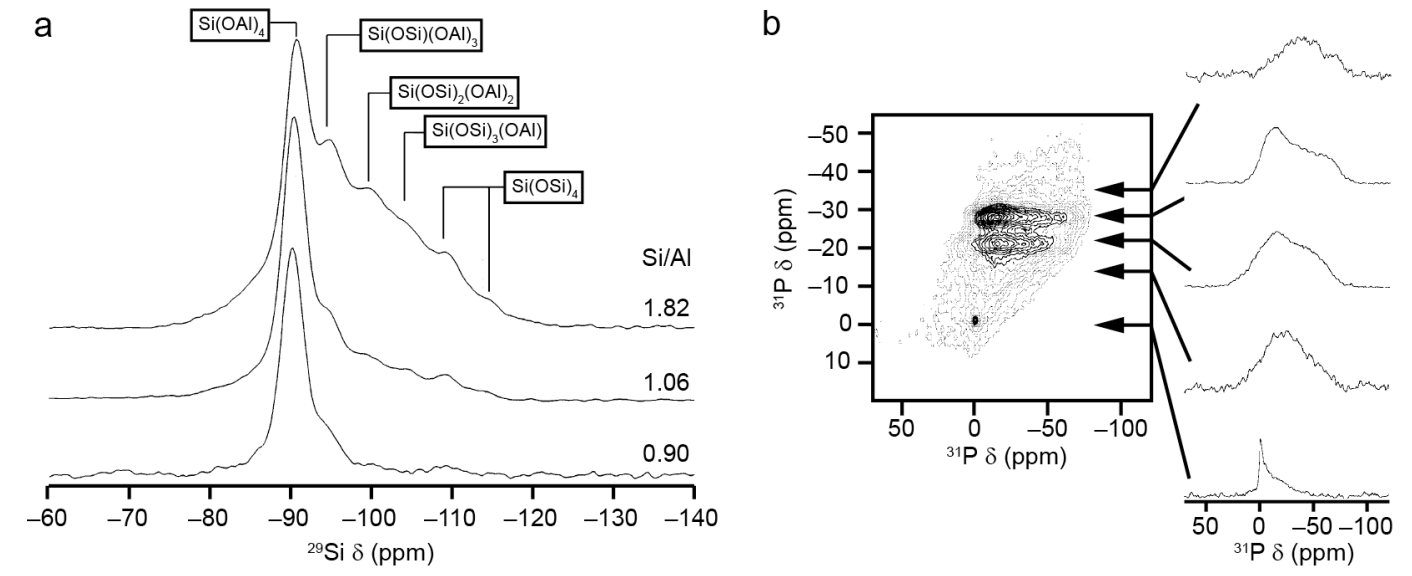
Figure 20

a ${ }^{13} \mathrm{C}\left\{{ }^{15} \mathrm{~N}\right\}$ REDOR curves
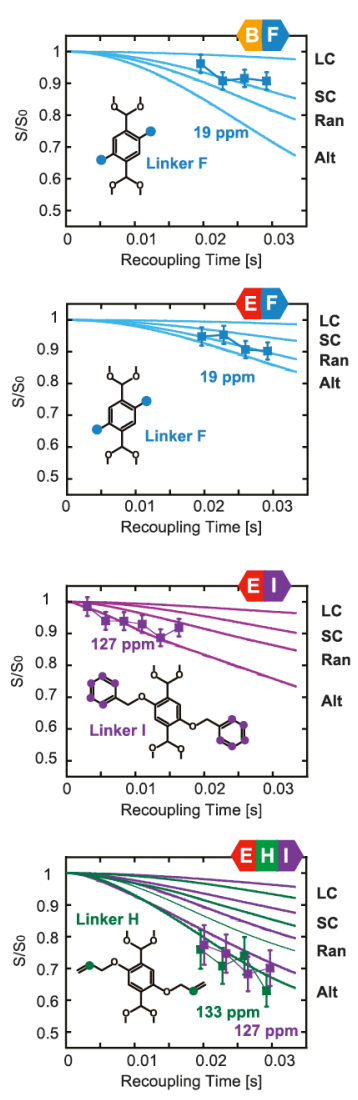

Scenarios of linker apportionment
in MTV-MOF-5 materials
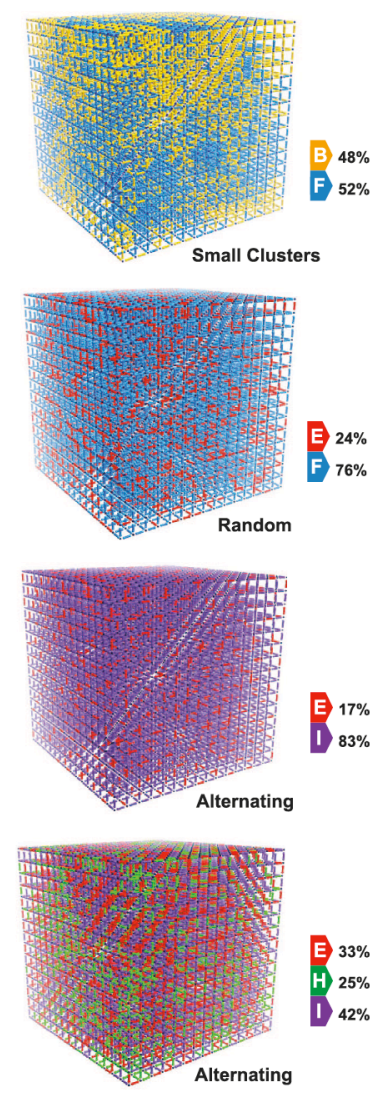

b

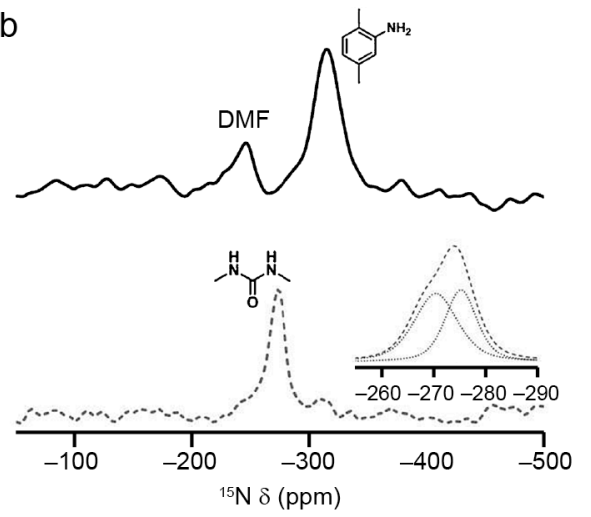

C
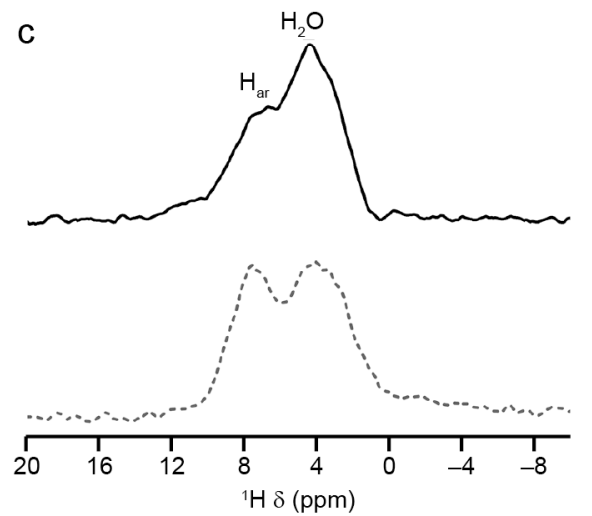


\section{Figure 21}
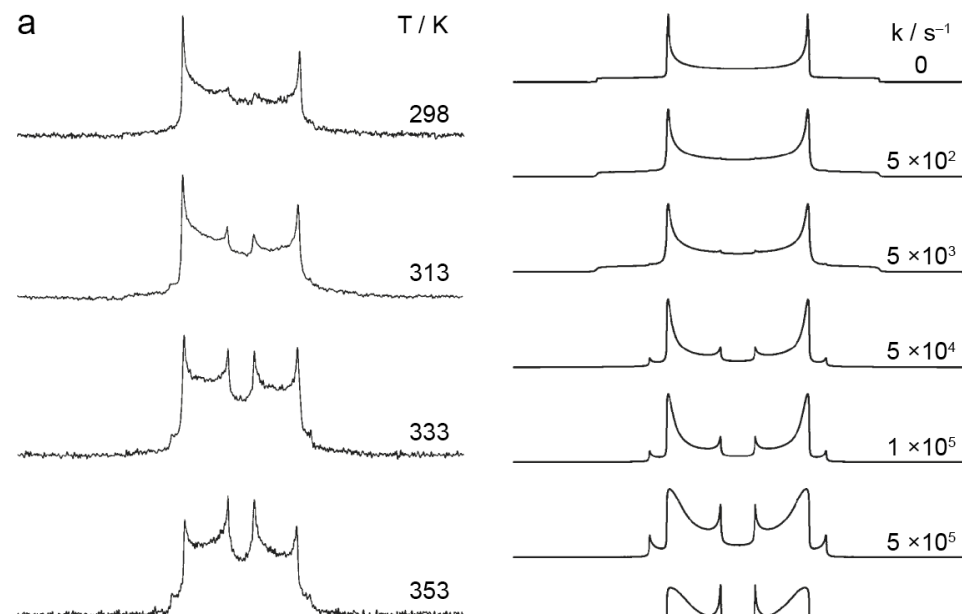

b fast rotation
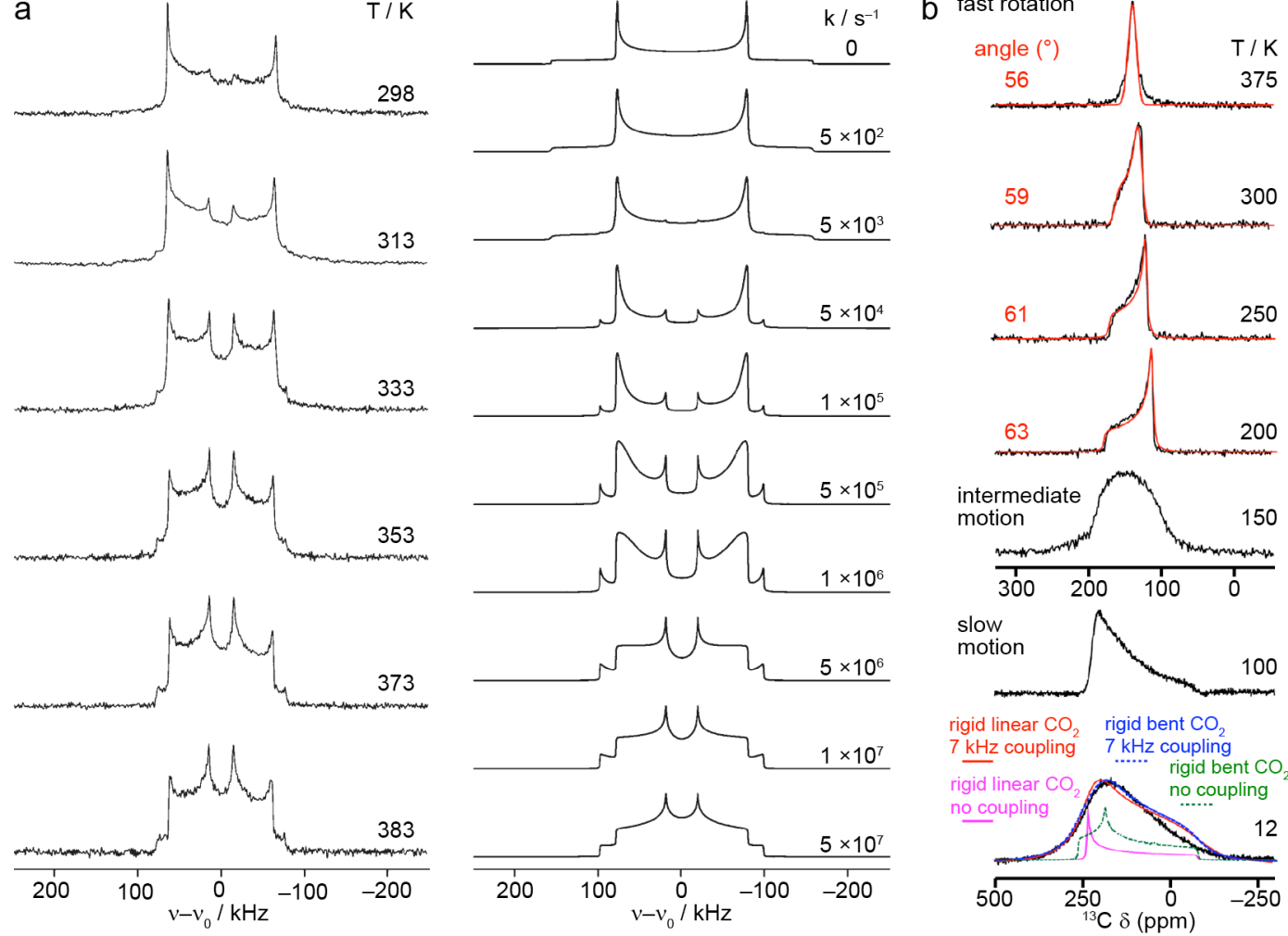

C exp sim (wobbling + hopping) sim (wobbling only)

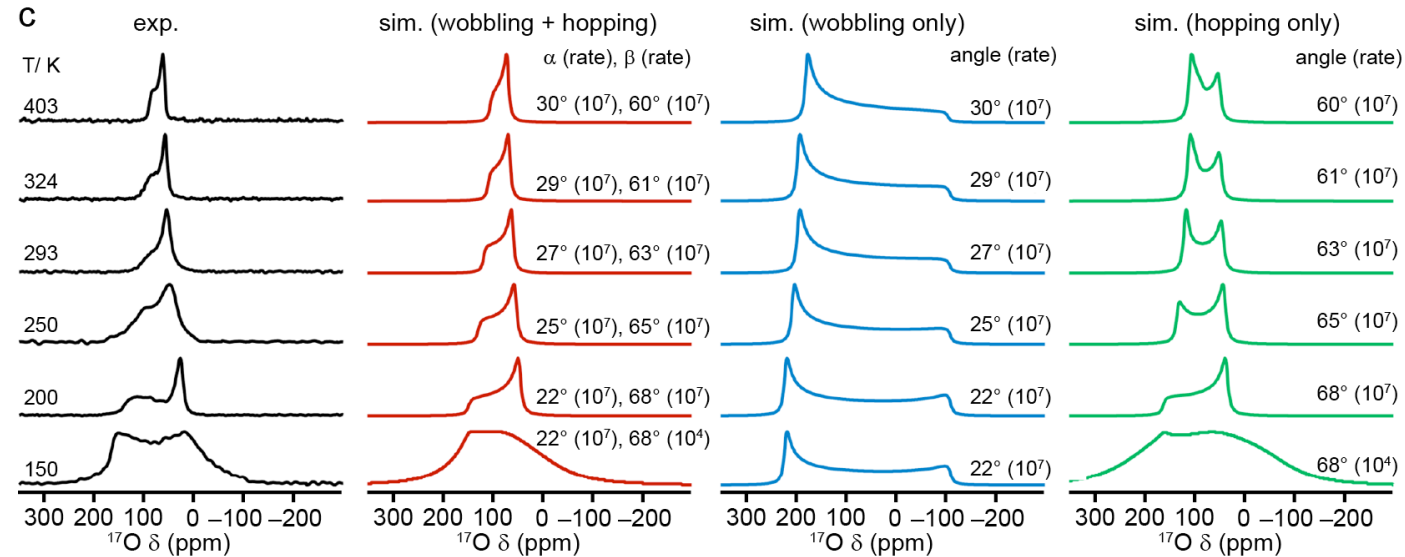


Figure 22

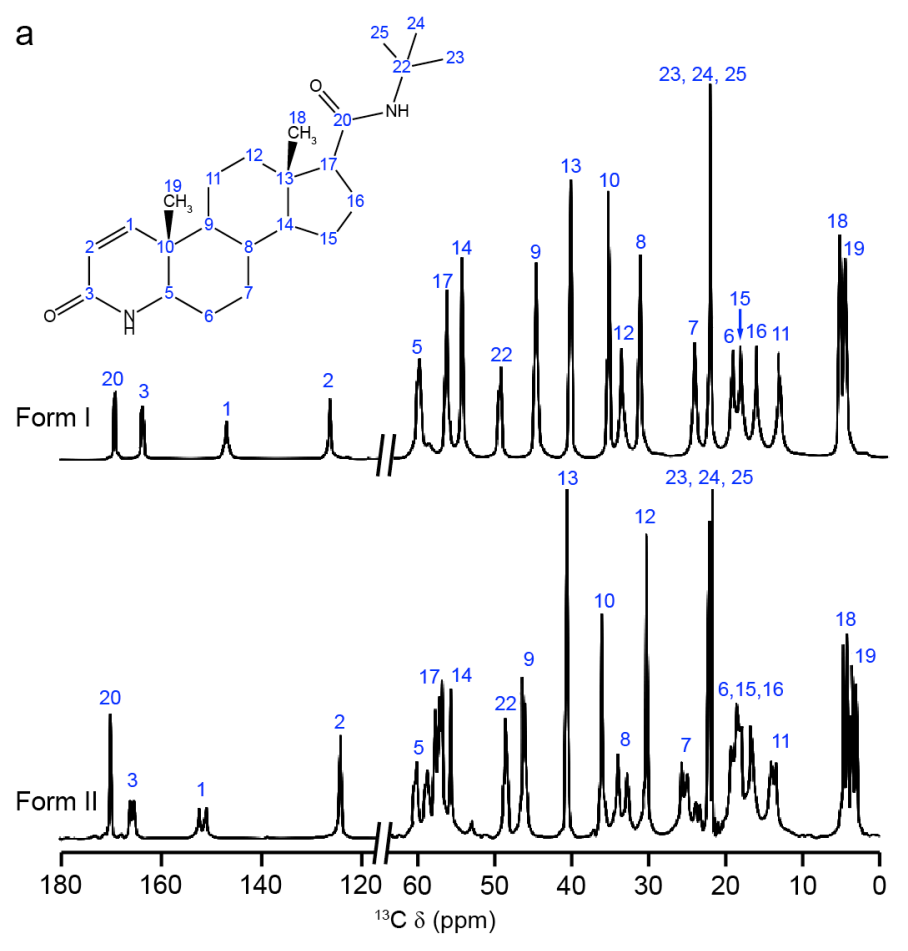

b

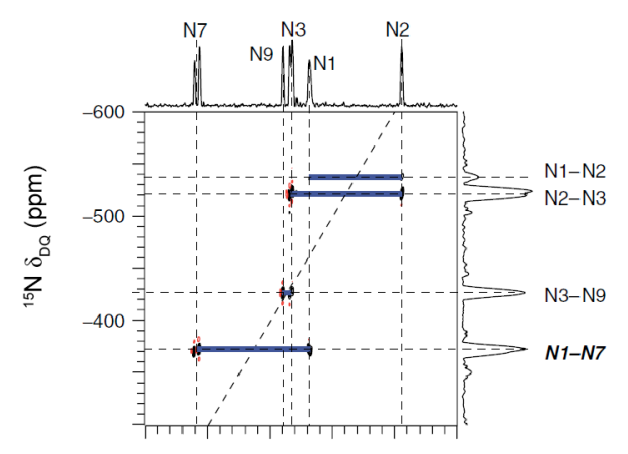

c
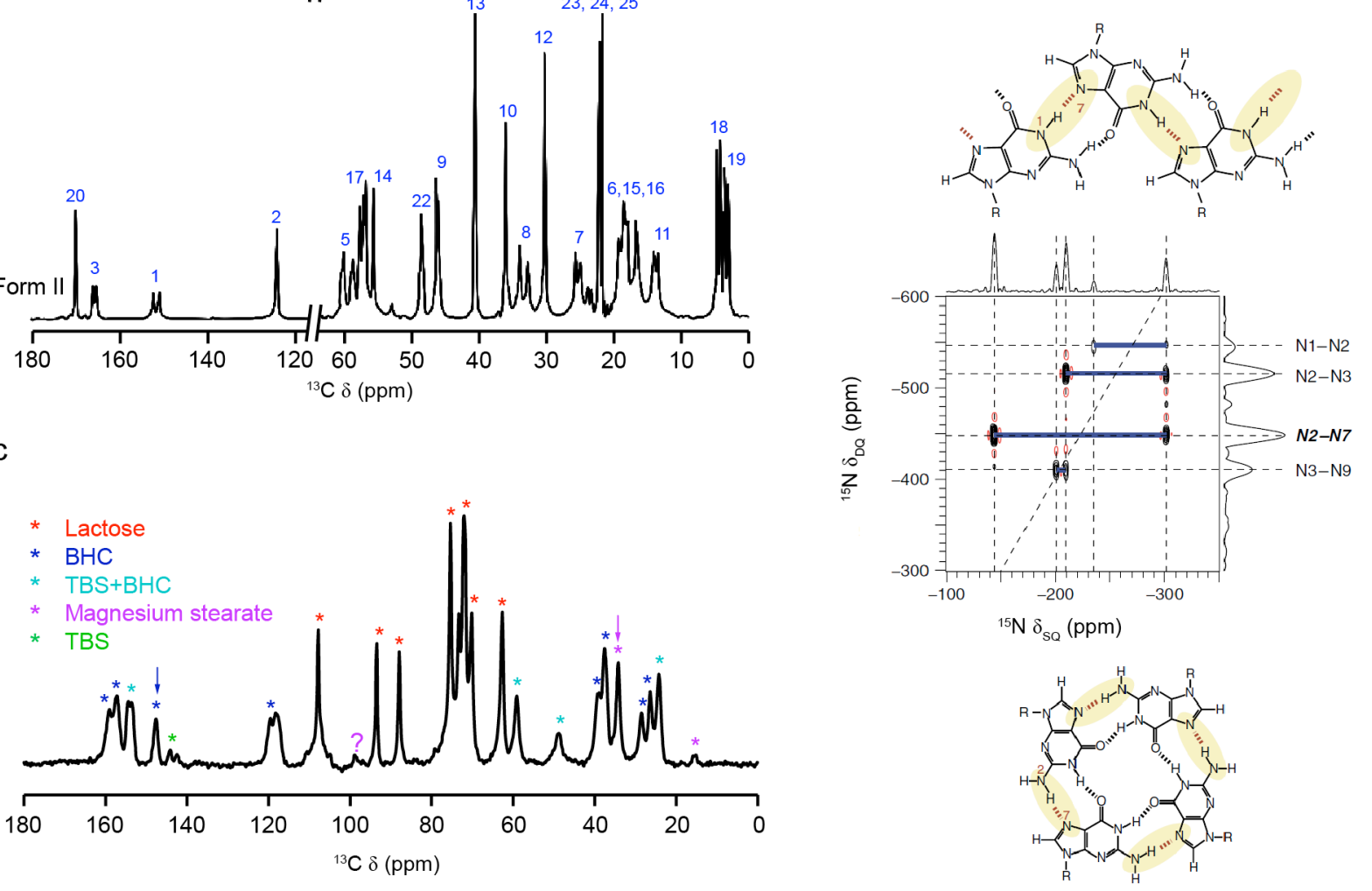
Figure 23

a

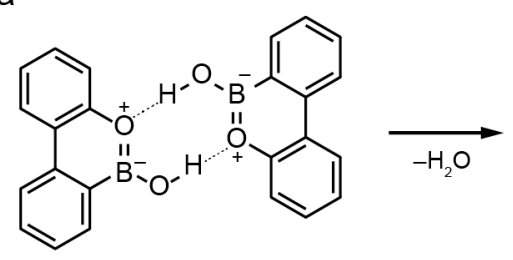

C

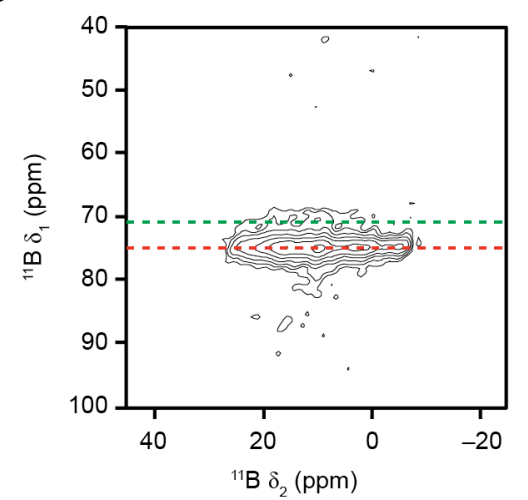

b<smiles>c1ccc2c(c1)O[B-](O[B-]1c3ccccc3-c3ccccc31)c1ccccc1-2</smiles>

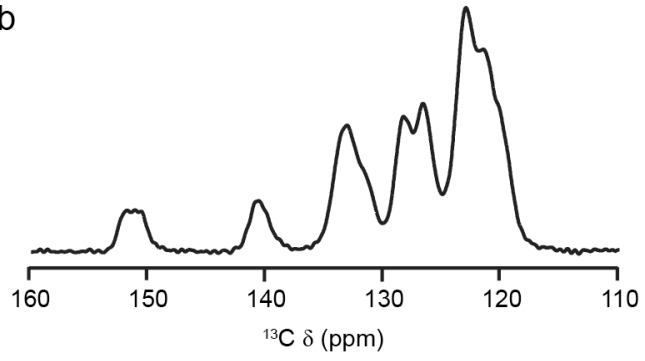

d

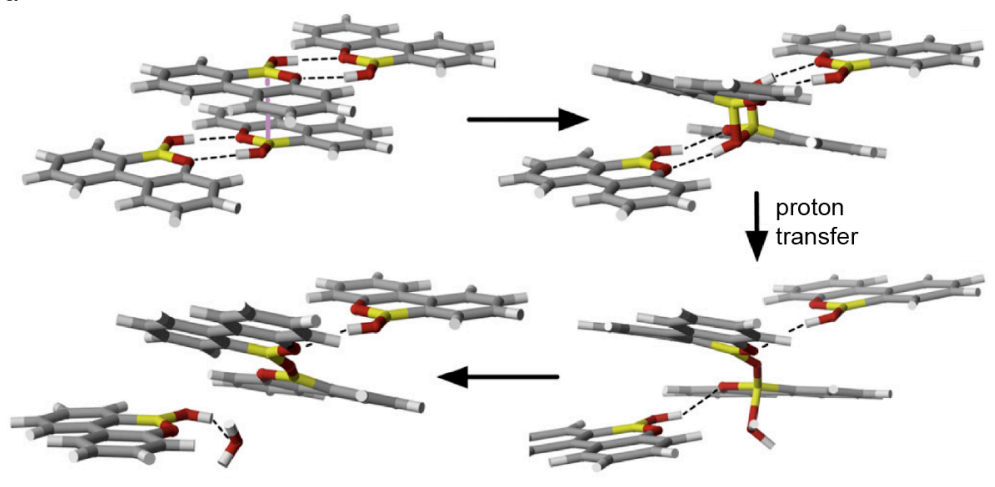


Figure 24
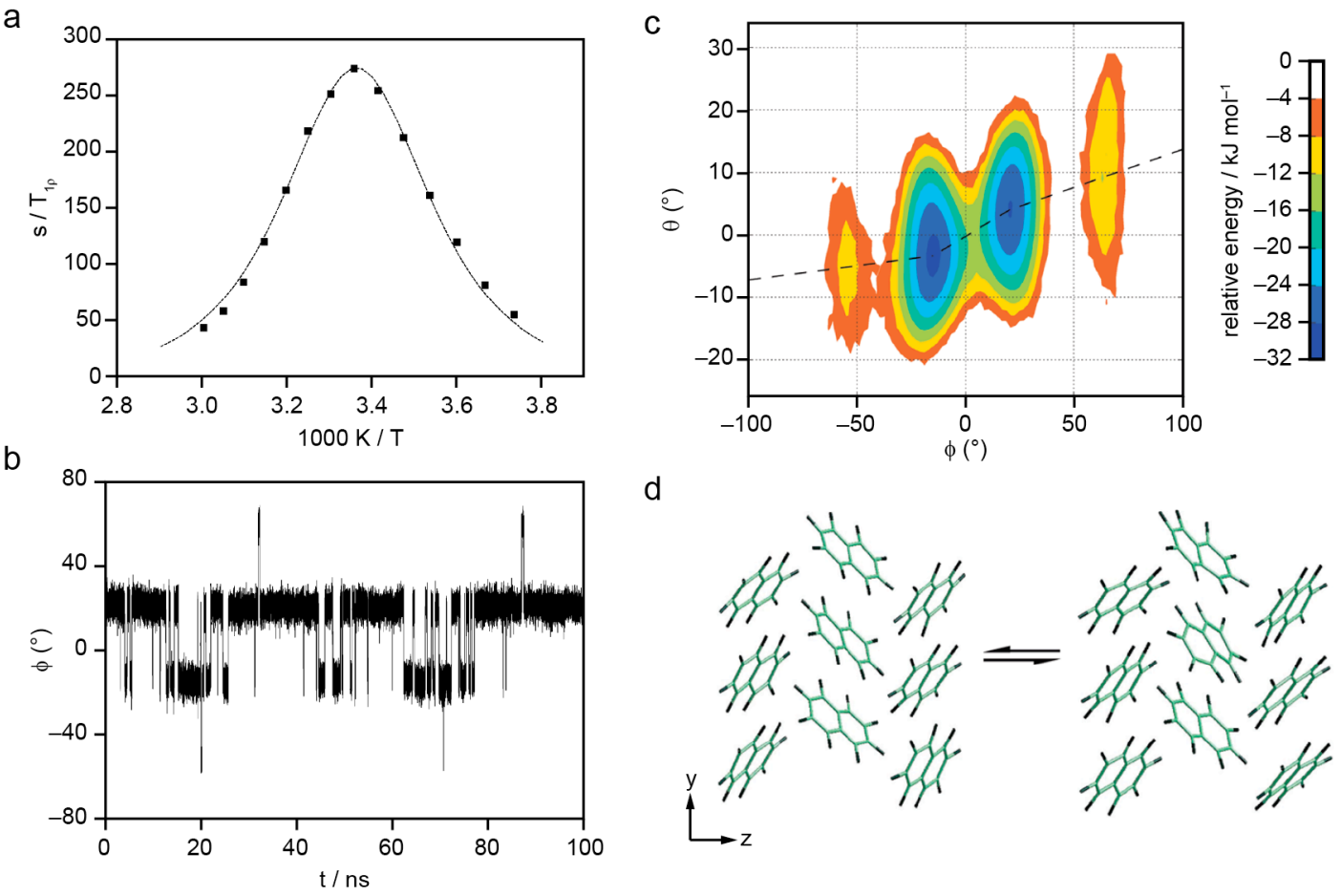

d

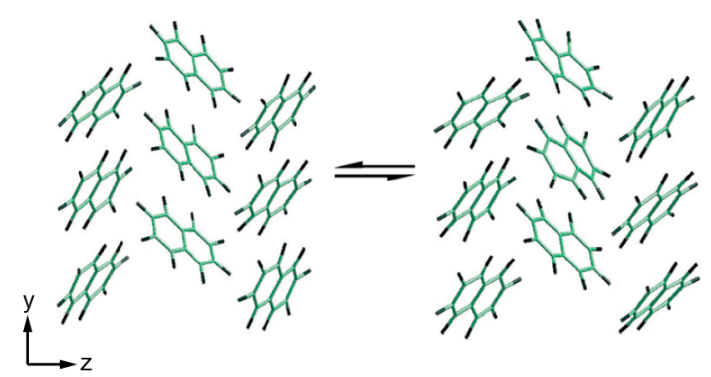




\section{Figure 25}

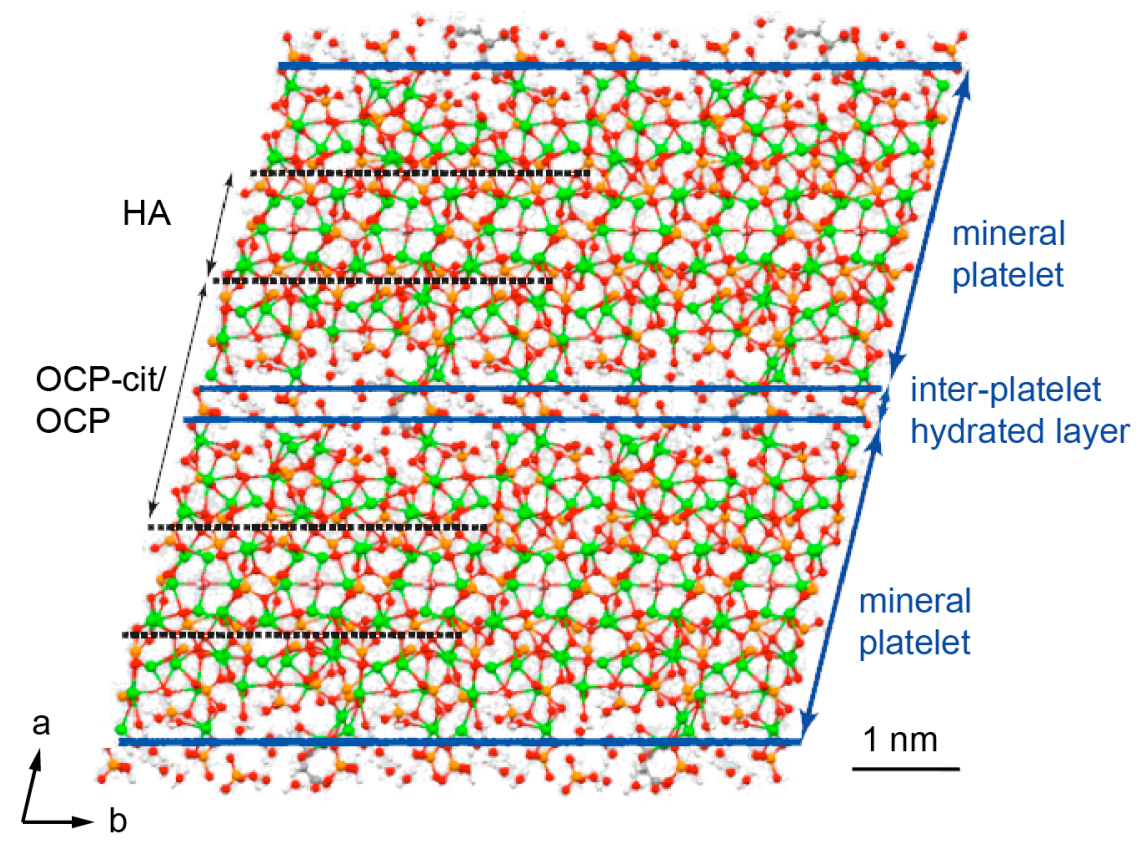




\section{Figure 26}

a

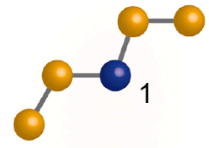

2
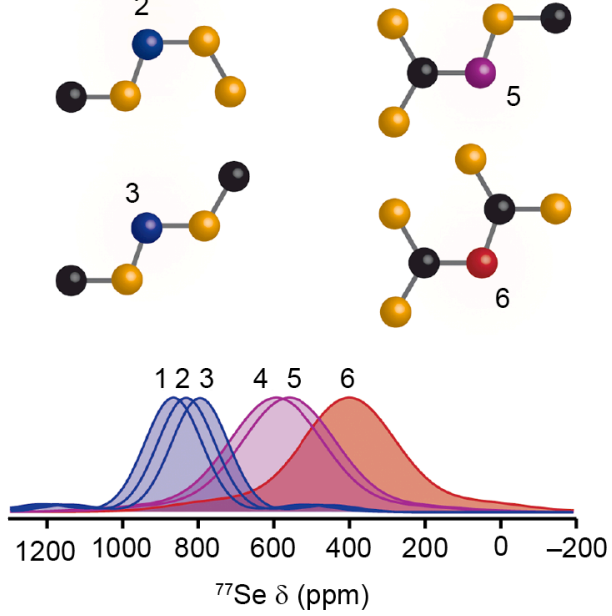

c

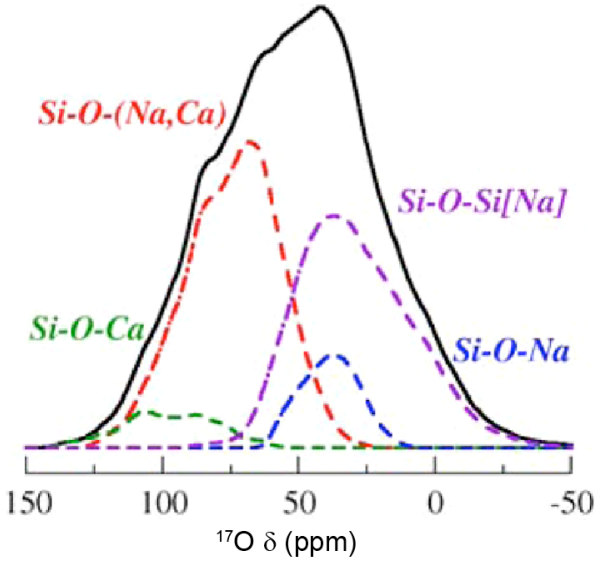

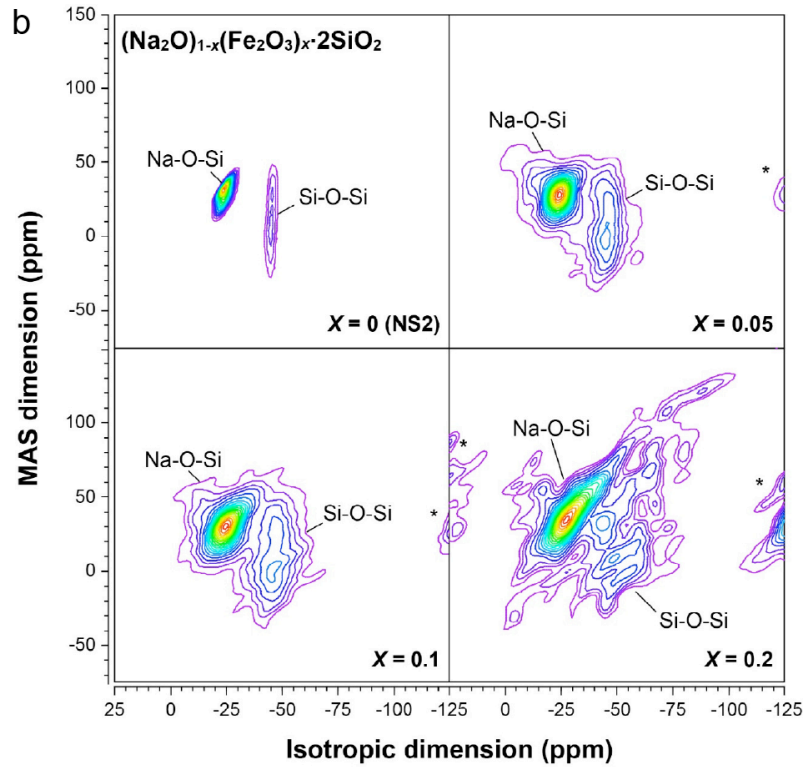

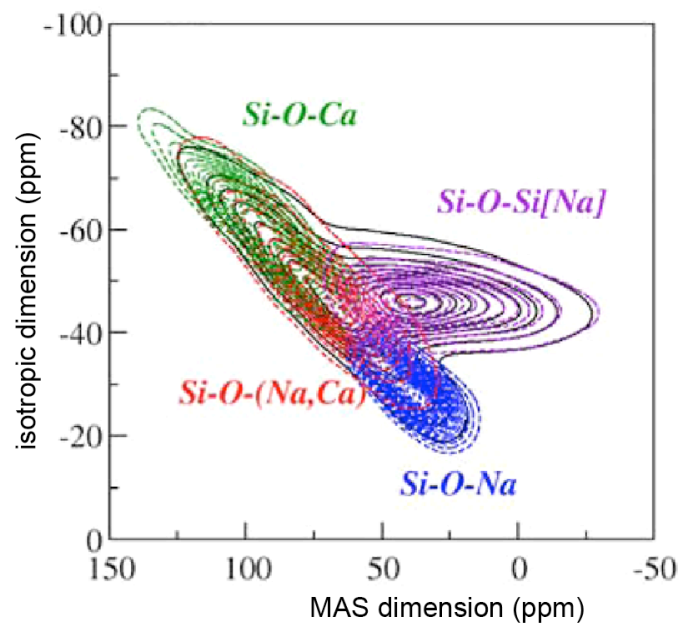




\section{Figure 27}
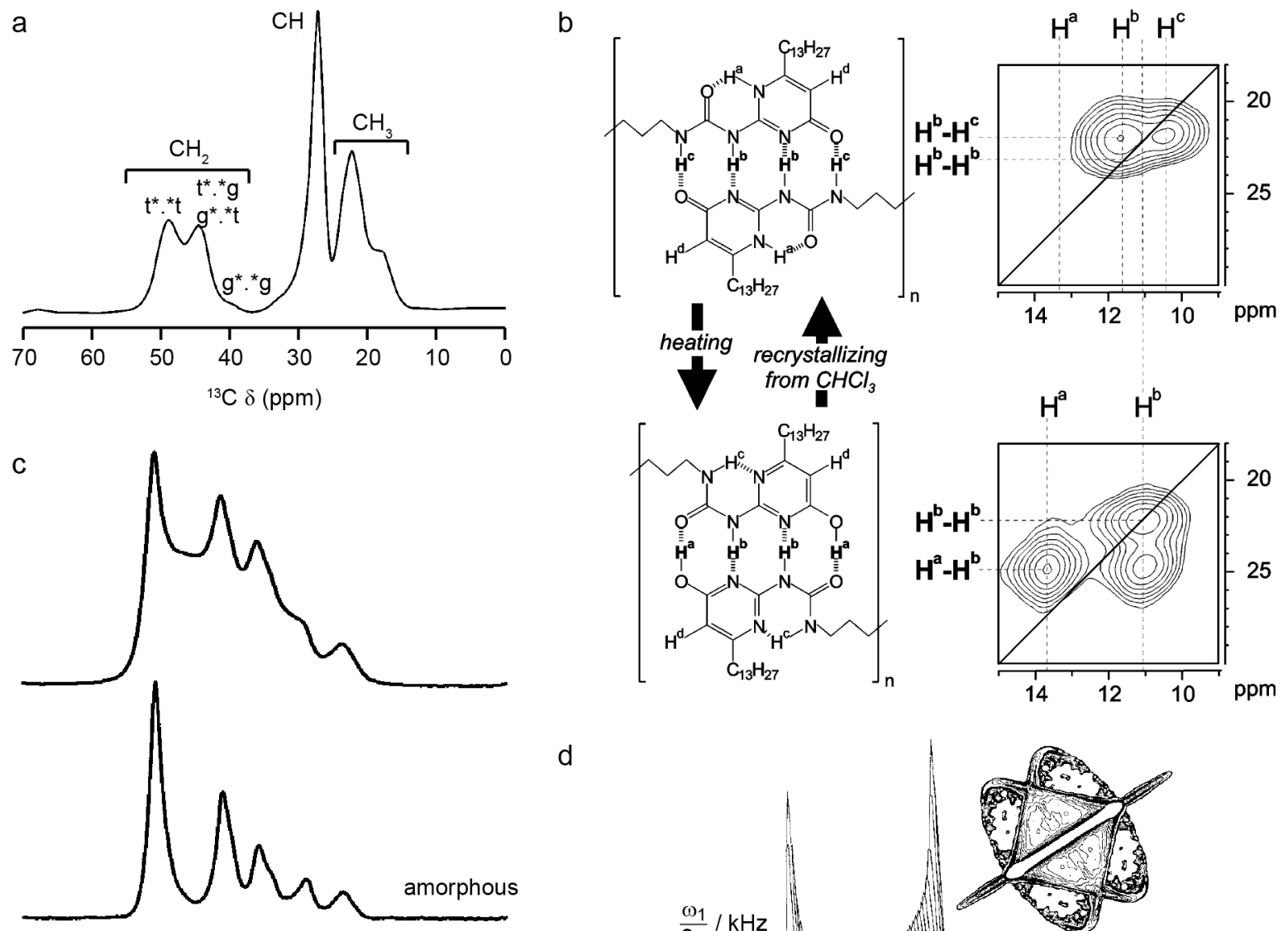

d
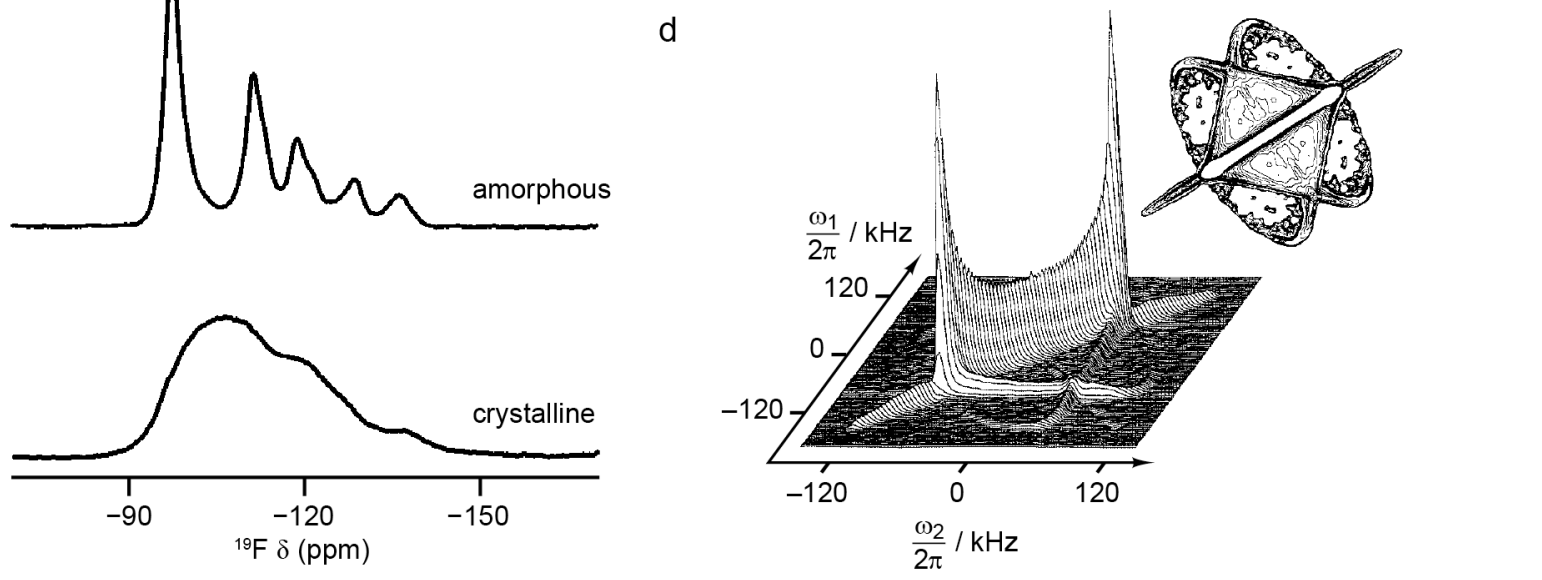